\title{
Framing health communication messages
}

Citation for published version (APA):

van 't Riet, J. P. (2009). Framing health communication messages. [Doctoral Thesis, Maastricht University]. Datawyse / Universitaire Pers Maastricht. https://doi.org/10.26481/dis.20091016jr

Document status and date:

Published: 01/01/2009

DOI:

10.26481/dis.20091016jr

Document Version:

Publisher's PDF, also known as Version of record

\section{Please check the document version of this publication:}

- A submitted manuscript is the version of the article upon submission and before peer-review. There can be important differences between the submitted version and the official published version of record.

People interested in the research are advised to contact the author for the final version of the publication, or visit the DOI to the publisher's website.

- The final author version and the galley proof are versions of the publication after peer review.

- The final published version features the final layout of the paper including the volume, issue and page numbers.

Link to publication

\footnotetext{
General rights rights.

- You may freely distribute the URL identifying the publication in the public portal. please follow below link for the End User Agreement:

www.umlib.nl/taverne-license

Take down policy

If you believe that this document breaches copyright please contact us at:

repository@maastrichtuniversity.nl

providing details and we will investigate your claim.
}

Copyright and moral rights for the publications made accessible in the public portal are retained by the authors and/or other copyright owners and it is a condition of accessing publications that users recognise and abide by the legal requirements associated with these

- Users may download and print one copy of any publication from the public portal for the purpose of private study or research.

- You may not further distribute the material or use it for any profit-making activity or commercial gain

If the publication is distributed under the terms of Article $25 \mathrm{fa}$ of the Dutch Copyright Act, indicated by the "Taverne" license above, 


\section{Framing Health Communication Messages}

Jonathan Pieter van 't Riet 
(C) Jonathan van 't Riet

Cover design: Bilbo Schickenberg

ISBN: 9789052788517

The studies presented in this thesis were performed within the School of Public Health and Primary Care (CAPHRI) which participates in the research school CARE, Netherlands School of Primary Care Research, accredited by the Royal Netherlands Academy of Arts and Sciences.

This research was funded by ZonMW - the Netherlands Organisation for Health Research and Development (grant number 6100.0005).

Financial support of the Kurt Lewin Institute for the publication of this thesis is gratefully acknowledged. 


\section{Framing Health Communication}

\section{Messages}

\section{Proefschrift}

ter verkrijging van de graad van doctor aan de Universiteit Maastricht, op gezag van de Rector Magnificus, Prof. mr. G.P.M.F. Mols, volgens het besluit van het College van Decanen, in het openbaar te verdedigen op vrijdag 16 oktober om 14:00 uur

$$
\text { door }
$$

Jonathan Pieter van 't Riet

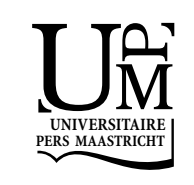




\section{Promotor}

Prof. dr. H. de Vries

Copromotor

Dr. R.A.C. Ruiter

Beoordelingscommissie

Prof. dr. N.K. de Vries (voorzitter)

Prof. dr. G. Kok

Prof. dr. L. Lechner (Open Universiteit)

Dr. R.M. Meertens

Dr. A.K. Üskül (University of Essex, United Kingdom) 
Also, we assume that wisdom is rather the knowledge that is preferred for the sake of itself and for the sake of knowing, than the knowledge that is preferred for the sake of its results; and that wisdom is rather the knowledge that leads than the knowledge that provides services.

Aristotle, Metaphysics

He, whose wisdom exceeds his works, to what may he be likened? To a tree whose branches are numerous but whose roots are few. The wind comes along and uproots it and sweeps it down.

Rabbi Eleazar Ben Azariah, The Wisdom of the Fathers

All the rocket ships are climbing through the sky, the holy books are open wide, the doctors working day and night, but they'll never ever find that cure for love

Leonard Cohen, Ain't No Cure for Love 



\section{Contents}

Part 1: Persuasive Effects of Message Framing 1

Chapter 1 Theoretical Background 3

Chapter 2 Investigating Message-Framing Effects in the Context of a 15 Tailored Intervention Promoting Physical Activity

Part 2: Underlying Mechanisms 27

Chapter 3 Distinct Pathways to Persuasion: The Role of Affect in 29 Message-Framing Effects

Chapter 4 What Difference Does a Frame Make? Investigating the 47 Influence of Affective Reactions to Skin Cancer Detection Messages

$\begin{array}{ll}\text { Part 3: Moderating Variables } & 61\end{array}$

Chapter 5 The Influence of Self-Efficacy on the Effects of Framed 63 Health Messages

Chapter 6 Self-Efficacy Moderates Message-Framing Effects: The Case 75 of Skin-Cancer Detection

Chapter 7 Examining the Influence of Self-Efficacy on Message- 85 Framing Effects: Reducing Salt Consumption in the General Population

Chapter 8 Investigating the Effects of Message Framing in High-and Low-Threat Information

General Discussion

Footnotes

References

Appendices

Summary

Samenvatting

Thank you 



\section{Part 1}

\section{Persuasive Effects of Message Framing}





\section{Chapter 1: Theoretical Background}

Cardiovascular diseases and cancers are the main causes of mortality in many Western countries. Because these and many other diseases are largely the result of unhealthy behaviours (WHO, 2002; 2003), an important goal of health education is to encourage and motivate people to engage in healthful and diseasepreventive behaviours. But how do we persuade people to behave in a healthy way? To reach this objective, investigators have studied the factors that can contribute to successful persuasive efforts. Among other things, they have investigated whether arousing fear is an effective instrument for persuasion (Ruiter, Abraham, \& Kok, 2001; Witte \& Allen, 2000), whether arousing guilt can be effective (O'Keefe, 2002), what the effects of argument strength and source expertise are (Petty \& Cacioppo, 1986), and whether communications that are individually tailored to the recipient are more persuasive than generic communications (De Vries \& Brug, 1999; Noar, Benac, \& Harris, 2007). In the research that is presented in the present thesis, we focused on the effects of the way healthpromoting information is framed. ${ }^{1}$

Health-promoting communications usually stress the value of healthy behaviour, but they can do this in several ways. In particular, the message can be framed in terms of the benefits of engaging in the behaviour (gain frame) or in terms of the costs of failing to engage in the behaviour (loss frame). Research has shown that these frames can have a different persuasive impact, even when the different frames are applied to objectively equivalent information (see for instance Gerend \& Cullen, 2008; Rothman \& Salovey, 1997). Unfortunately, studies show inconsistent results with regard to which type of framing is more persuasive. The results of several studies have suggested that gain-framed information is more persuasive than loss-framed information (e.g., Cox, Cox, \& Zimet, 2006; Detweiler, Bedell, Salovey, Pronin, \& Rothman, 1999; Reinhart, Marshall, Feeley, \& Tutzauer, 2007), the results of other studies have suggested that loss-framed information is more persuasive (e.g., Banks, Salovey, Greener, \& Rothman, 1995; Meyerowitz \& Chaiken, 1987; Rivers, Salovey, Pizarro, Pizarro, \& Schneider, 2005; Schneider et al., 2001a) and some studies have reported no differential effects (Brug, Ruiter, \& Van Assema, 2003; Jones, Sinclair, Rhodes, \& Courneya, 2004; Steffen, Sternberg, Teegarden, \& Shepherd, 1994; see O'Keefe \& Jensen, 2006 for a meta-analysis). Therefore, we tested the persuasive impact of gain- and loss-framed messages in nine experiments. The aim of this research was twofold. First, we tried to shed some light on the inconsistent findings in the message-framing literature, by investigating the underlying mechanisms of framing effects. Because finding no differential effects of message framing might obscure the fact that different, but equally potent mechanisms might underlie the effects of gain- and loss-framed information (Shen \& 
Dillard, 2007), we investigated whether we could identify the pathways through which gain- and loss-framed information exert their influence on persuasion. In other words, we tried to identify mediators of the persuasive effects of framed messages. Second, to explore the reasons for the inconsistent findings in the message framing literature, research is needed that investigates under which circumstances gain- or loss-framed information is more persuasive. Research investigating possible moderating variables that can explain differences in the effects of gain- and loss-framed messages is indispensable to deepening our understanding of message-framing effects on persuasion. The second aim of our research, therefore, was to investigate whether we could identify potential moderators of the persuasive effects of framed health-promoting messages.

In the remainder of this introduction, we will give an overview of the message framing literature, outline the theoretical background of our studies and introduce our hypotheses. Finally, we will give a brief overview of the studies that were conducted.

\section{Overview of the literature}

Most of the studies that investigated the effects of message framing were concerned with identifying possible moderating variables of message framing effects. In fact, the first studies on message framing (Meyerowitz \& Chaiken, 1987; Rothman \& Salovey, 1997; Rothman, Salovey, Antone, Keough, \& Drake Martin, 1993) hypothesized that whether gain- or loss-framed information was more persuasive depended on the function of the advocated behaviour. More specifically, in these studies the authors made a distinction between behaviours that served to prevent ill health, such as exercise or quitting smoking, and behaviours that served to detect an illness, like skin self-examination or obtaining a mammography. They hypothesized that gain-framed information would be more persuasive when advocating prevention behaviours and that loss-framed information would be more persuasive when advocating detection behaviours. Two other noteworthy variables that have been proposed as moderators are the perceived risk that is associated with the advocated behaviour (Latimer, Salovey, \& Rothman, 2007), and mode of information processing (Maheswaran \& Meyers Levy, 1990; Meyers Levy \& Maheswaran, 2004).

\section{Prevention and detection behaviours}

According to Prospect Theory (Kahneman \& Tversky, 1979; 1984; Tversky \& Kahneman, 1981), people respond differently to information about the consequences of a behavioural decision depending on whether the same consequences are presented as gains or losses. For instance, in a well known study, Tversky and Kahneman (1981) asked participants to imagine the outbreak of a 
terrible 'Asian disease', that, according to calculations, would kill 600 US citizens if left untreated. Next, they told participants that two medical programmes had been developed to counter the disease. It was calculated that Programme A would save 200 people, whereas the outcomes of Programme B were less certain: Programme B had a one-third chance of saving all 600 people, and a twothird chance of saving no-one. In this example, most participants preferred the saver Programme A over the riskier Programme B. Another group of participants were given the same dilemma, only now the options were framed differently. This second group was told that, as a consequence of Programme A, 400 people would die, whereas Programme B would result in a one-third chance that no people would die and a two-third chance that all people would die. Interestingly, in this group of participants the majority preferred the riskier Programme B over the saver Programme A, suggesting that people are risk-aversive when considering gains, but risk-seeking when considering losses.

Several authors have applied these findings to health behaviour and health education (Banks, Salovey, Greener, \& Rothman, 1995; Meyerowitz \& Chaiken, 1987; Rothman \& Salovey, 1997; Rothman et al., 1993). They have reasoned that framing health education messages can have different effects dependent on the nature of the behaviour being advocated. In particular, these authors have distinguished behaviours that serve to prevent a health problem (e.g., using sunscreen or exercising) from behaviours that serve to detect a health problem (e.g., skin self-examination or obtaining a mammography). They argue that prevention behaviours are relatively risk-less, because they reduce the risk of illness. For this reason, gain-framed information may be more effective for promoting prevention behaviours, because gain-framed information motivates people to adopt riskless alternatives. It has also been argued that people perceive detection behaviours as inherently risky because detection behaviours involve the possibility of finding out that one is ill (Meyerowitz \& Chaiken, 1987). Because people are risk-seeking when considering losses, loss-framed information should be more likely to motivate people to engage in risky detection behaviours (Meyerowitz \& Chaiken, 1987; Rothman \& Salovey, 1997; Rothman et al., 1993).

Several authors, however, have pointed out that there are some fundamental problems with the above reasoning. Assuming that detection behaviours are generally perceived as more dangerous or entailing more negative outcomes than prevention behaviours is questionable (Cox et al., 2006; Kuhberger, 1998). Whereas people may perceive detection behaviours as having potential unfavourable outcomes on the short run, it is hard to see why people should perceive detection behaviour as dangerous on the long run (Cox et al., 2006). In addition to these theoretical objections, a recent meta-analysis showed that for behaviours serving to detect an illness, gain- and loss-framed messages were not differentially persuasive (O'Keefe \& Jensen, 2006). For behaviours 
serving to prevent an illness, a small advantage of gain-framed information was found, although subsequent analyses revealed that this effect only occurred in a limited amount of studies on dental health (O'Keefe \& Jensen, 2007).

\section{Perceptions of behaviour as safe or risky}

In response to these and similar objections, Latimer and colleagues (2007) have argued that Prospect Theory could still provide an adequate framework for studying message framing. However, they argue that researchers should focus less on whether the recommended behaviour serves to prevent or detect illness but more on the way the recipient perceives the behaviour. Similarly, Schneider and colleagues (2001b) have suggested that, even though smoking cessation clearly is a prevention behaviour, some people might perceive quitting smoking as entailing many costs. Consequently, such people might be more responsive to a loss-framed message. Some studies have found support for the hypothesis that loss-framed information is more persuasive for people who perceive the recommended behaviour as more risky (Apanovitch, McCarthy, \& Salovey, 2003; Kiene, Barta, Zelenski, \& Cothran, 2005; Lee \& Aaker, 2004).

One fundamental problem still remains, however, and this concerns the central theoretical notion that perceived risk influences the effects of framed messages. When Rothman and Salovey (1997) proposed that detection behaviours entailed a higher risk than prevention behaviours, they referred to the 'risk of receiving significant, unpleasant information' (p. 5). This sense of the word 'risk' is conceptually close to the colloquial meaning of the word, which is related to concepts such as 'danger' and 'the possibility of something bad' (Cox et al., 2006; O'Keefe \& Jensen, 2006). In contrast, when Prospect Theory mentions risk, it refers to uncertainty of outcomes. People who are 'risk-seeking' in this sense of the word prefer an uncertain option that entails both positive and negative possibilities to a certain, but mediocre, option. Because it is the possibility of positive outcomes that helps explain Kahneman and Tversky's results, making use of the colloquial meaning of 'risk', from which positive outcomes are absent, is not likely to yield the same reliable effects (Cox et al., 2006; Kuhberger, 1998; Levin, Schneider, \& Gaeth, 1998). Thus, the concept of 'risk' can be operationalized in different ways and this can explain some of the inconsistencies in the message-framing literature.

In sum, although several authors have argued that gain-framed information is more persuasive for prevention behaviours and loss-framed information is more persuasive for detection behaviours (Meyerowitz \& Chaiken, 1987; Rothman \& Salovey, 1997; Rothman et al., 1993), empirical findings have not always been in accordance with this view and theoretical objections have been raised. As a result, other potentially important moderating variables have been proposed. One notable example is mode of information processing. 


\section{Mode of processing}

According to dual process accounts of persuasion (Chaiken, Liberman, \& Eagly, 1989; Petty \& Cacioppo, 1986; for an overview see Chaiken \& Trope, 1999), persuasive messages are processed in one of two modes: heuristically or systematically. ${ }^{2}$ With heuristic processing attention is paid to surface features of the message (e.g., the expertise of the message source, the length of the message). These surface features can work as heuristic cues to facilitate persuasion. With systematic processing, attention is paid to particular details in message content. Several factors can facilitate people's mode of information processing, among which are personal involvement with the issue (Chaiken et al., 1989; Petty \& Cacioppo, 1986), a person's mood (Schwarz, Bless, \& Bohner, 1991), and a person's need for cognition (Cacioppo, Petty, Feinstein, \& Jarvis, 1996). Besides person characteristics, certain aspects of the message can also influence whether message processing is systematic or heuristic. One message aspect that receives much attention in the health-education literature is the extent to which a message is individually tailored to the recipient (Kreuter, Farrell, Olevitch, \& Brennan, 2000). Higher personal relevance, and thus higher attention, as well as stronger cognitive processing of the health message have been proposed as important mechanisms for the increased effectiveness of computer-tailored health messages over traditional non-tailored communications (e.g. Brug, Oenema, \& Campbell, 2003; Ruiter, Kessels, Jansma, \& Brug, 2006; for a recent metaanalysis see Noar et al., 2007).

Some authors have argued that when people process persuasive messages heuristically, people use positive information in the message as a heuristic, responding more favourable towards more positive messages. Thus, under conditions of heuristic processing, gain-framed information should be more persuasive than loss-framed information (Maheswaran \& Meyers Levy, 1990; Meyers Levy \& Maheswaran, 2004; Steward, Schneider, Pizarro, \& Salovey, 2003). Indeed, some studies have found that for individuals with a low need for cognition (i.e., individuals who are likely to process information heuristically; Cacioppo et al., 1996), gain-framed information was more persuasive than loss-framed information, while for individuals with a high need for cognition (i.e., individuals who are likely to process information systematically) gain- and loss-framed information had no differential effects (Steward et al., 2003; see Donovan \& Alleh, 2000 for similar findings).

It has also been proposed that, when processing information systematically, people tend to focus more on negative information, because of a 'negativity bias' (Maheswaran \& Meyers Levy, 1990; Meyers Levy \& Maheswaran, 2004). The negativity bias refers to the assumption that, because people perceive the world as predominantly positive, negative information will trigger more attention than factually equivalent positive information (Fiske, 1980; Kanouse, 1984; Lau, 1985; Wright \& Weitz, 1977). The results of several studies have 
suggested that loss-framed information might indeed be more persuasive than gain-framed information under conditions of systematic processing. Maheswaran and Meyers-Levy (1990), for instance, increased personal involvement with the issue of coronary heart disease in half of their student participants by telling them that 'even people under 25 are at risk for coronary heart disease'. The other half of their participants were told that coronary heart disease mainly affects senior citizens. The result of their study suggest that personal involvement resulted in higher levels of systematic processing, and that, under conditions of high involvement, loss-framed information was more persuasive, whereas under conditions of low involvement, gain-framed information was more persuasive (see also Meyers Levy \& Maheswaran, 2004; Shiv, Britton, Payne, Mick, \& Monroe, 2004).

Although the mode-of-processing hypothesis can account for the above findings, other studies have yielded results that are incompatible with the notion that gain-framed information is more persuasive under conditions of heuristic processing and loss-framed information is more persuasive under conditions of systematic processing. Millar and Millar (2000), for instance, found that when participants were highly involved in the issue gain-framed information was more persuasive than loss-framed information and Zhang and Buda (1999) found that participants with a low need for cognition were more effectively persuaded by a loss- rather than a gain-framed message. Thus, the influence of mode of processing on the effects of framed messages is currently less than clear (see also Rothman et al., 1993).

\section{The present thesis}

As is clear from the literature outlined above, message-framing research has mainly investigated the moderating influence of potentially important variables. We have discussed the function of the promoted behaviour, perceptions of the behaviour as safe versus risky, and mode of processing as three particularly noteworthy examples. Other examples are approach/avoidance orientation (Mann, Sherman, \& Updegraff, 2004; Sherman, Mann, \& Updegraff, 2006; Updegraff, Sherman, Luyster, \& Mann, 2007), regulatory focus (Aaker \& Lee, 2001; Cesario, Grant, \& Higgins, 2004; Lee \& Aaker, 2004), perceived behavioural norms (Blanton et al., 2001), ambivalence (Broemer, 2002), ease of imagination (Broemer, 2004), mood (Wegener, Petty, \& Klein, 1994), previous risky behaviour (Gerend \& Shepherd, 2007), source credibility (Jones, Sinclair, \& Courneya, 2003; Zhang \& Buda, 1999), level of education (Smith, 1996), selfdiscrepancies (Tykocinski, Higgins, \& Chaiken, 1994), and intention to engage in the recommended behaviour (Wong \& McMurray, 2002). However, evidence for the importance of these moderators has sometimes been limited to a single 
study (e.g., Smith, 1996), or to a single health behaviour (for instance flossing; Mann et al., 2004: Sherman et al., 2006; Updegraff et al., 2007). In other instances, researchers have relied on student samples only, limiting the generalizability of the results (Aaker \& Lee, 2001; Cesario et al., 2004; Lee \& Aaker, 2004).

In the present thesis, we aimed to contribute to the literature in two ways. First, because finding no differential effects of message framing might obscure the fact that different, but equally potent mechanisms might underlie the effects of gain- and loss-framed information (Shen \& Dillard, 2007), we report on three studies that aimed to identify the pathways through which gain- and loss-framed information may exert their influence on persuasion. In other words, we tried to identify mediators of the effects of framed messages. Second, we report on the results of five studies that investigated the role of two factors that might have a moderating influence on the effects of framed messages, but are overlooked in the literature until now: the recipients' self-efficacy to perform the recommended behaviour and the level of threat that is conveyed by the message. To increase the generalizability of the results, we aimed to test these hypotheses in both student and non-student samples, using framed messages on several health behaviours.

\section{Underlying Mechanisms: The role of affect}

As we have mentioned above, recent meta-analyses provide strong evidence that gain- and loss-framed messages do not affect psychological and behavioural outcomes in a consistent and predictable way across all populations (O'Keefe \& Jensen, 2006; 2007). However, finding no differential effects of message framing might obscure the fact that different, but equally potent mechanisms might underlie the effects of gain- and loss-framed information (Shen \& Dillard, 2007). To gain more insight in these mechanisms, it is important to try to identify potential mediators of the effects of gain- and loss-framed messages. Therefore, in the research that is presented in this thesis, we aimed to identify important mediators of message-framing effects. We hypothesized that both positive affect as a result of gain-framed information and negative affect as a result of loss-framed information can contribute positively to persuasion. To the extent that these two variables contribute equally to persuasion, gain- and lossframed messages are bound to have no differential effects on persuasive outcome measure such as attitudes, intentions and behaviour.

As Shen and Dillard (2007) point out, gain-framed health promoting messages focus on beneficial outcomes, whereas loss-framed health promoting messages focus on costs. For this reason, gain-framed information might elicit more positive affect than loss-framed information, and loss-framed information might elicit more negative affect than gain-framed information. The limited research that exists on the relationship between framing and affect is consistent 
with this logic (Chang, 2005; Cox et al., 2006; Cox \& Cox, 2001; Millar \& Millar, 2000; Schneider et al., 2001a; Shen \& Dillard, 2007).

Several scholars have studied the effects of message-induced affect on persuasion. One theoretical account of the relationship between message-induced affect and persuasion stems from marketing and consumer psychology and is called the 'affect transfer hypothesis' (MacKenzie, Lutz, \& Belch, 1986). According to this account, advertisements that evoke positive affect result in a positive attitude towards the ad, which in turn results in a positive attitude towards the advertised brand. A meta-analysis of persuasion studies in the field of marketing showed support for this hypothesis (Brown, Homer, \& Inman, 1998).

Negative affect has also drawn attention from persuasion scholars. Numerous studies tested the effects of fear appeals on measures of attitude, intention and behaviour (for overviews see Eagly \& Chaiken, 1993; Ruiter et al., 2001). A fear appeal is a persuasive message attempting to arouse fear to promote precautionary motivation and self-protective action (Rogers, 1983). Metaanalyses of the fear-persuasion relationship have shown that evoking fear can enhance a message's persuasive power (Mongeau, 1998; Witte \& Allen, 2000). With regards to framed messages, the persuasive effects of both positive and negative affect imply that gain- and loss-framed messages exert their influence on persuasion to a large extent through quite different substrates (positive versus negative affect; see also Cacioppo \& Berntson, 1994).

In the present thesis, we report the results of three experiments that investigated whether gain-framed information gives rise to greater levels of positive affect than loss-framed information, whereas loss-framed information gives rise to greater levels of negative affect. The experiments further tested the hypothesis that both positive and negative affect can increase persuasion.

\section{Moderators: Self-efficacy and message threat}

Self-efficacy. As mentioned above, research shows that loss-framed information evokes greater levels of negative affect than gain-framed information. We hypothesized that increased negative affect can contribute to persuasion and, in fact, operates as a mediator of framing effects. This is in line with metaanalyses of the relationship between fear and persuasion, which suggest that increased fear enhances message effectiveness (Mongeau, 1998; Witte \& Allen, 2000). Several scholars, however, propose that the effect of fear-arousing messages is contingent on the level of the recipient's perceived efficacy (Ruiter et al., 2001; Witte, 1992). Perceived efficacy consists of response efficacy (the perception that the recommended action can avert the threat; Rogers, 1975) and selfefficacy (confidence in one's personal ability to perform the recommended behaviour; Bandura, 1986). According to Witte (1992), at least some level of perceived threat is needed for recipients to be motivated to pay attention to the message. However, Witte also argues that perceived threat only results in persuasion 
when recipients perceive that they are able to avert the threat, i.e., when their perceived efficacy is high. If perceived efficacy is low, recipients will engage in 'fear control', which consists of efforts to reduce the unpleasant experience of fear by means of avoidance or denial of the message.

Thus, whereas we aimed to investigate the hypothesis that negative affect can contribute to persuasion (see above), an additional possibility would be that perceived efficacy moderates the effects of gain- and loss-framed messages. Loss-framed messages have been found to evoke a greater sense of threat than gain-framed messages (Cox \& Cox, 2001; Shen \& Dillard, 2007), and this greater sense of threat might in turn increase a message's persuasiveness (Witte $\&$ Allen, 2000). This, however, seems particularly likely when recipients have a high perceived efficacy. In this case, loss-framed messages may be more persuasive than gain-framed messages. For recipients with little confidence that the threat can be averted, loss-framed information is unlikely to be more persuasive than gain-framed information, because the greater sense of threat that is conveyed by loss-framed information might give rise to defensive processing (Stephenson \& Witte, 1998; Witte, 1992). As a result, gain- and loss-framed messages may not have different effects on persuasion. In the present research we tested these assumptions, focusing on self-efficacy instead of response-efficacy for two reasons. First, we used framed messages in which we stressed the beneficial (harmful) consequences of (not) performing the recommended behaviour. In a sense, then, we already addressed response efficacy in these messages. This would make a potential influence of response efficacy hard to interpret. Second, in some cases (e.g., smoking cessation) knowledge about the beneficial effects of healthy behaviour is widespread. Therefore, we did not expect large differences in response-efficacy among participants. In the present thesis then, we report on three experiments that investigated whether participants' self-efficacy could moderate the effects of gain- and loss-framed messages.

Message threat. It is conceivable that message framing effects are dependent on the content of the framed message. If message content interacts with message framing, this can have important implications for practice. For instance, if research would show that low-threat information is particularly persuasive when it is gain-framed as opposed to loss-framed, these findings could result in clear recommendations for health-care professionals designing low-threat messages. However, this possibility has received little attention until now (Rothman, Bartels, Wlaschin, \& Salovey, 2006). In one study that did focus on message content, Gerend and Cullen (2008) investigated the effects of gain- and lossframed messages about alcohol use, stressing either the long-term consequences or the short-term consequences of (not) drinking. They found that the gainframed message was more persuasive, but only when the message stressed the short-term consequences of not drinking. In this thesis, we tried to expand this line of research, also focusing on the influence of message content. We report on 


\section{Chapter 1}

three experiments that investigated the effects of framing, using information that either had a high- or a low-threat content. Thus, we combined message-framing research and fear-appeal research. Following a previous study (Ruiter, Kok, Verplanken, \& Van Eersel, 2003), we hypothesized that gain-framed information would be more persuasive for low-threat information whereas loss-framed information would be more persuasive for high-threat information.

\section{Overview of the thesis}

\section{Part 1: Persuasive Effects of Message Framing}

Above we have discussed the message-framing literature and outlined the theoretical background of the performed studies. We concluded that gainand loss-framed messages generally do not affect persuasive outcomes differently. As a first test of this hypothesis, we conducted an Internet-based study into the effects of gain- and loss-framed messages, which is reported in Chapter 2. We used framed information promoting physical activity in Study 2, recruiting 787 adult participants through a health-related website. Our expectation for this study was that gain- and loss-framed information would not have different effects on persuasion. In Part 2 of the thesis, we investigate our hypotheses regarding mediating variables and in Part 3 we investigate our hypotheses regarding moderating variables. To increase the generalizability of our results, we used gain- and loss-framed messages on five different health behaviours (physical activity, salt consumption, skin self-examination, smoking, and alcohol use).

\section{Part 2: Underlying Mechanisms}

In Part 2 we report on our studies investigating the mediating role of positive and negative affect. Chapter 3 reports on two studies that were conducted with the hypothesis that gain-framed information gives rise to higher levels of positive affect than loss-framed information, that loss-framed information gives rise to higher levels of negative affect, and that positive and negative affective reactions could both contribute to persuasion. We used information promoting physical activity as the stimulus materials in Study 3.1, recruiting 100 student participants in the university restaurant. In Study 3.2, which took place in our laboratory, we used framed messages promoting a low-salt diet, recruiting 129 students to participate.

Study 4, reported in Chapter 4, had the same hypotheses as Study 3.1 and 3.2, but additionally tested the influence of approach and avoidance orientation on the effects of message framing. Study 4 also took place in our laboratory and used a student sample of 168 participants. 


\section{Part 3: Moderating Variables}

In Part 3 we report on six studies investigating the moderating role of self-efficacy and message threat. Chapter 5 reports on a study that investigated the hypothesis that self-efficacy would moderate framing effects, such that lossframed messages would be more persuasive, but only for recipients with high self-efficacy levels. We used framed anti-smoking messages as the stimulus materials in Study 5, recruiting 539 adult participants on markets and fairs throughout the Netherlands.

As in Study 5, the hypotheses of Study 6 and Study 7 (reported in Chapter 6 and 7 respectively) was that self-efficacy would moderate framing effects, such that loss-framed messages would be more persuasive, but only for recipients with high self-efficacy levels. In Study 6 we provided 124 student participants with framed messages promoting skin self-examination. In Study 7 we used framed messages promoting a low-salt diet, recruiting 575 participants through an Internet panel.

Chapter 8 reports on two studies that investigated whether the level of threat that is conveyed in the message could influence the effects of gain- and loss-framed messages in a student population, hypothesizing that a gain frame might be more persuasive for low-threat information whereas a loss frame might be more persuasive for high-threat information. Study 8.1 took place in the university restaurant, used a student sample $(n=98)$ and investigated the effects of framed information promoting physical activity. For Study 8.2, 99 students were recruited to come to the laboratory to participate. For this study we used framed messages discouraging alcohol use. ${ }^{3}$

\section{General Discussion}

In the General Discussion, we will summarize the findings from these studies, address major limitations, implications of the results and we will provide some concluding thoughts. 



\title{
Chapter 2: Investigating Message-Framing Ef- fects in the Context of a Tailored Intervention Promoting Physical Activity ${ }^{4}$
}

\begin{abstract}
Health-promoting messages can be framed in terms of the gains associated with healthy behaviour or the losses associated with unhealthy behaviour. It has been argued that gain-framed messages promoting physical activity are more effective than loss-framed messages but empirical findings are inconsistent. Also, no previous studies investigated the effects of gain- and loss-framed messages in the context of a tailored intervention to promote physical activity. In this study, we provided participants $(\mathrm{N}=787)$ with computer-generated tailored feedback concerning their physical activity levels. We investigated whether gainand loss-framed messages promoting physical activity affected information acceptance, attitude, intention and behaviour differently. The results showed that gain-framed messages resulted in stronger intentions to be physically active than loss-framed messages. This did not result in a significant increase in actual physical activity, however, as measured by a three-month follow-up assessment. For information acceptance and attitude, a non-significant advantage of gainframed messages was found. All effects had small effect sizes. These results suggest that, whereas gain-framed information might be more persuasive than loss-framed information when it comes to promoting physical activity, the differences between gain- and loss-framed messages are likely to be small.
\end{abstract}

\section{Introduction}

Insufficient physical activity has been known to have a detrimental effect on blood pressure, body fat, glucose metabolism and obesity (Department of Health, 2004; WHO, 2002). Obesity, in turn, is associated with an increased risk of future morbidity and mortality, and sizeable decreases in disability-free life expectancy (Peeters et al., 2003). Unfortunately, a large number of people in the western world do not engage in sufficient levels of physical activity. In the Netherlands, it is estimated that less than half of the adult population meets the recommendation for sufficient physical activity (Hildebrandt, Ooijendijk, Stiggelbout, \& Hopman-Rock, 2004), which states that healthy adults should be physically active for at least thirty minutes on at least five days of the week (Kemper, Ooijendijk, \& Stiggelbout, 2000). Therefore, effective interventions are needed to motivate people to adopt a healthier lifestyle. In the present study we investigated whether the effectiveness of such interventions can be increased by mes- 
sage framing. Persuasive health messages can be framed in terms of the benefits of engaging in healthy behaviour (gain frame), or in terms of the costs of failing to engage in healthy behaviour (loss frame). The present study investigated whether gain-framed information would be more effective than loss-framed information in promoting physical activity.

Empirical studies have shown that gain- and loss-framed messages can have different effects on individuals' self-protective motivation and action, even when the persuasive information in gain- and loss-framed health messages is factually equivalent (Rothman, Bartels, Wlaschin, \& Salovey, 2006; Rothman \& Salovey, 1997). One influential approach to the study of message framing has been to focus on the function of the recommended behaviour, making a distinction between behaviours that serve to prevent an illness (like physical activity or quitting smoking) and behaviours that serve to detect an illness (like skin selfexamination or obtaining a mammography). According to Rothman and Salovey (1997), people perceive disease-prevention behaviours as relatively safe, because they minimize the chance of falling ill. In contrast, people perceive diseasedetection behaviours as inherently risky because they entail the possibility of finding out that one is ill. Drawing from Prospect Theory (Kahneman, 2003; Kahneman \& Tversjy, 1984), Rothman and Salovey go on to suggest that gainframed information is more persuasive when advocating disease-prevention behaviours, because gain-framed information makes people risk-averse and thus more likely to engage in relatively safe disease-prevention behaviours. In contrast, they suggest that loss-framed information is more persuasive for diseasedetection behaviours, because loss-framed information makes people willing to take risks and thus more likely to engage in relatively risky disease-detection behaviours.

Inspired by Rothman and Salovey's reasoning, several previous studies have investigated the effects of framing for messages advocating physical activity. Most of these have hypothesized that gain-framed communications would be more effective than loss-framed communications. A study by Latimer and colleagues (2008a), for instance, found that a gain-framed message resulted in higher levels of physical activity than a loss-framed or a mixed gain- and lossframed message nine weeks after participants received the message. A second study by the same research group (Latimer et al., 2008b), however, indicated that, while participants judged the gain-framed message as more informative than the loss-framed message, no main effect of frame on behaviour at a twoweek follow-up was found. Other studies also yielded mixed findings. McCall and Ginis (2004) found that a gain-framed message resulted in higher levels of exercise over a three-month period than a loss-framed message or no-message, although only the difference between the gain-framed message condition and the no-message control condition was significant. Jones and colleagues (2003), on the other hand, found that a gain-framed message was only more effective when 
the message-source was highly credible. For messages that had a less credible source, there were no differences between the gain- and loss-framed message. A second study (Jones et al., 2004) failed to replicate these effects, finding no differential effects between the gain- and loss-framed message, either originating from credible or incredible sources.

These mixed findings make it difficult to arrive at clear recommendations for health-care professionals who wish to promote physical activity. In addition, a recent meta-analysis of the message-framing literature showed that gain-framed information seemed to have a small advantage over loss-framed information when encouraging disease-prevention behaviours, but this effect was only found in a limited amount of studies on dental health. For other preventive behaviours, no difference was found between gain- and loss-framed information (O'Keefe \& Jensen, 2007). Thus, in contrast to Rothman and Salovey's (1997) reasoning, it is currently unclear how health-care professionals should frame persuasive messages aimed at encouraging disease-prevention behaviours.

A major limitation of the literature, however, is the fact that messageframing studies have predominantly investigated the effects of gain- and lossframed leaflets, pamphlets or other printed materials (e.g., Broemer, 2002; Broemer, 2004; Jones et al., 2003; McCall \& Ginnis, 2004; Rothman, Martino, Bedell, Detweiler, \& Salovey, 1999; Van 't Riet, Ruiter, Werrij, \& De Vries, 2008). A small minority of studies have used framed information delivered by other means, such as telephone messages (Latimer et al., 2008b) or framed video clips (Schneider et al., 2001b). However, many health-education efforts nowadays make use of information that is in some way computer-tailored to the individual recipient. Computer tailoring is a promising cost-effective health education technique that enables the development of individualized messages and is able to reach large populations against relatively low costs per person (Brug, Oenema, \& Campbell, 2003; Dijkstra \& De Vries, 1999; Rimer \& Kreuter, 2006). As a result, tailored messages are more likely to be read, understood, remembered, discussed with others, and are rated as better, saved more often and evaluated as more interesting than non-tailored messages (Dijkstra \& De Vries, 1999; Kreuter, Farrell, Olevitch, \& Brennan, 2000; Ruiter, Kessels, Jansma, \& Brug, 2006).

One study investigated the effects of message framing in combination with ethnic targeting (Schneider et al., 2001a), but to our knowledge, no study has investigated the effects of gain- and loss-framed messages in the context of a tailored intervention to promote healthy behaviour. This is disappointing because computer tailoring is a well established and often used means of transmitting health education information (Noar, Benac, \& Harris, 2007). To foster our understanding of framing effects it is important to investigate which effects gainand loss-framed messages have on recipients in the context of a tailored intervention to promote healthy behaviour. 
In sum, we investigated whether gain-framed information would be more effective than loss-framed information in promoting physical activity, as proposed by Rothman and Salovey (1997) and found by Latimer and colleagues (2008a). To increase external validity, we tested the effects of gain- and lossframed messages in the context of an on-line tailored intervention to promote physical activity. Outcome measures of our study were information acceptance, attitude and intention measured directly after participants received the framed information. Furthermore, physical activity levels were assessed at a three-month follow-up.

\section{Method}

\section{Recruitment}

A webpage was created providing the public with information about health and healthy behaviour (www.health-alert.nl). To increase interest for the website, we advertised the website on local television and local newspapers in the province of Limburg in The Netherlands. To recruit participants for our study, we created a hyperlink on the Health-Alert webpage called 'physical activity check' which lead to a physical activity expert-system, where people could check whether their physical activity levels were in line with recommendations. As noted above, Dutch recommendations with regards to physical activity state that healthy adults should be physically active for at least thirty minutes on at least five days of the week (Kemper, Ooijendijk, \& Stiggelbout, 2000). In addition to checking whether they were sufficiently active, participants could enrol in the present study. In the introduction to the study, participants were told that upon participation they would be eligible to win a $€ 50$ price. Only adults were eligible to participate in the study, the minimum age required for participation being 18 years.

\section{Procedure and Design}

After participants entered the study, they were informed about the study and were asked whether they consented that their answers would be used for scientific purposes. Next, participants' demographics, current physical activity levels and intention to be physically active for thirty minutes on at least five days a week were assessed. Participants were then provided with a short message about the Dutch recommendations for physical activity. After participants were informed about these recommendations, they received tailored feedback concerning their current physical activity levels. This tailored feedback informed them about whether or not they met the recommendations for physical activity, and encouraged them to either increase their physical activity or maintain their (already sufficient) current level of physical activity. Next, they received a persua- 
sive communication about physical activity, which was either gain- or lossframed. This framed communication followed the computer-tailored feedback, but was not tailored to the individual recipient. After reading the persuasive communication, participants completed the dependent measures. Three months later, participants received an email that contained a measure of physical activity. In case of non-response, participants were sent a reminder email once, one week later. The present study used a one-factorial (frame: gain versus loss) betweenparticipants design.

\section{Pre-test questionnaire}

Demographics. We assessed gender, age, ethnicity, and education. In addition we asked participants to indicate how they learned about the physical activity check ( 1 = through a search engine, e.g., Google; 2 = through a link on another website; 3 = through an advertisement in a newspaper; $4=$ through family, friends, co-workers; 5 = through local television).

Assessment of baseline physical activity. Physical activity levels were assessed using the short version of the International Physical Activity Questionnaire (Craig et al., 2003). One item asked participants to indicate on how many days during the past weeks they had engaged in vigorous physical activity (e.g., exercising). One item assessed the time participants typically spent being vigorously physically active on such a day (in minutes). The two measures were multiplied to arrive at a total score of vigorous physical activity per week. Next, one item asked participants to indicate on how many days during the past week they had engaged in moderate physical activity (e.g., gardening, cycling). One item assessed the time participants typically spent being moderately physically active on such a day (in minutes). The two measures were multiplied to arrive at a total score of moderate physical activity per week. Finally, vigorous and moderate physical activity were added up to arrive at a total score of physical activity during the past week (Craig et al., 2003).

Baseline intention. To assess baseline intention to be physically active, one item asked participants to indicate to what extent they agreed with the statement ' $I$ intend to be physically active for at least thirty minutes on at least five days of the week' on a 7-point scale $(1=$ totally disagree; $7=$ totally agree $)$.

\section{Persuasive Message}

The framed persuasive communication consisted of a short message about either the positive consequences of being sufficiently physically active or the negative consequences of not being sufficiently physically active. The gainframed message contained 330 words and the loss-framed message contained 326 words. The full texts are available in Appendix A. 


\section{Outcome measures}

Manipulation checks. To investigate whether the manipulation of frame had been successful, we assessed positive and negative affective reactions to the framed communications. Based on previous research, it was expected that the gain-framed communication would result in higher levels of positive affect and that the loss-framed message would result in higher levels of negative affect (e.g., Cox \& Cox, 2001; Shen \& Dillard, 2007; for similar manipulation checks see Schneider et al., 2001a). Two items assessed positive affective reactions to the information (positive affect) by asking participants to indicate the extent to which they thought the information made them feel happy $(1=$ very happy; $7=$ not at all happy $)$ and relieved $(1=$ very relieved; $7=$ not at all relieved $)$. Scores were reversed and combined to create an average positive affect score $(\alpha=.78)$. Two items assessed negative affective reactions to the information (negative affect), assessing the extent to which participants thought the information made them feel sad $(1=$ very $\operatorname{sad} ; 7=$ not at all sad $)$ and afraid $(1=$ very afraid $7=$ not at all afraid). Scores were reversed and combined to create an average negative affect score $(\alpha=.82)$.

Intention. Three items were used to assess intention to be physically active. Two items asked participants to indicate whether they planned to be physically active for at least thirty minutes a day on at least five days of the week and whether they considered being physically active for at least thirty minutes a day on at least five days of the week $(1=$ definitely not; $7=$ definitely $)$. One item asked participants: 'how likely is it that you will be physically active for at least thirty minutes a day on at least five days of the week in the coming six months?' $(1=$ very unlikely; $7=$ very likely $)$. An average intention score was calculated $(\alpha$ $=.92)$.

Attitude. Five items were used to assess attitude towards physical activity, asking participants to indicate on semantic differentials the extent to which they rated engaging in at least thirty minutes of physical activity for at least five days of the week as: (1) very good to (7) very bad, (1) very important to (7) very unimportant, (1) very sensible to (7) not sensible at all, (1) very nice to (7) not at all nice, (1) a lot of fun to (7) no fun at all. Scores were reversed and then averaged to create an attitude score $(\alpha=.87)$.

Information acceptance. Five items assessed information acceptance by asking participants to indicate the extent to which they thought the information was relevant $(1=$ very relevant; $7=$ not at all relevant $)$, interesting $(1=$ very interesting; $7=$ not at all interesting $)$, objective $(1=$ very objective; $7=$ not at all objective), and exaggerated ( 1 = very exaggerated; $7=$ not at all exaggerated $)$. Furthermore, one item asked participants to indicate the extent to which participants agreed with the information $(1=I$ totally agree; $7=I$ totally disagree $)$. After we reversed the scores of all items except the exaggerated item, the scores 
on the five items were averaged to create an average information acceptance score $(\alpha=.81)$.

Physical activity. At the three-month follow-up, physical activity levels were assessed using the same procedure as in the pre-test questionnaire (i.e., using the IPAQ).

\section{Statistical analysis}

First, we investigated the demographic profile of the sample. Second, we performed attrition analyses to investigate whether drop-out could be predicted by condition or any of the variables assessed at baseline. Third, linear regression analyses tested the main effects of frame (coded as $0=$ loss frame, $1=$ gain frame) on positive affect, negative affect, information acceptance, attitude, intention, and behaviour. There were no differences between conditions with regards to baseline variables $(p s>.08$ ), but baseline physical activity and baseline intention were entered as covariates to increase statistical power. There were no interactions between frame and any of the baseline measures on the outcome measures $(p s>.14)$ suggesting that the influence of the covariates on the outcome measures are equal for both conditions of frame. The semi-partial correlation $(s r)$ was used as a measure of effect sizes, and was interpreted according to guidelines by Cohen (1992), stating that $s r=.10$ corresponds with a small effect size, $s r=.30$ corresponds with a medium effect size, and $s r=.50$ corresponds with a large effect size. We used the statistical package SPSS 15.0 for the analyses.

\section{Results}

\section{Participants}

In total, 787 people participated in the experiment. The sample consisted of $55.1 \%$ women $(n=434)$. Age ranged from 18 to 87 years, with a mean age of 46.3 years $(\mathrm{SD}=14.0)$. Most of the participants were native Dutch ( $87.0 \%$ versus $13.0 \%$ non-native), $42.7 \%$ of the participants $(n=336)$ had a high education level, $41.9 \%(\mathrm{n}=330)$ had a medium education level, and $15.4 \%(\mathrm{n}=$ 121) had a low education level. In the complex schooling system in the Netherlands, a low education level refers to primary or basic vocational school, a medium education level refers to secondary vocational school or high school, and a high education level refers to advanced vocational school or university. Of the entire sample, 39.6\% $(\mathrm{n}=307)$ indicated that they learned about the physical activity check on local television, while $25.8 \%$ (200) indicated that they learned about it on related websites and $19.2 \%(n=149)$ learned about it through advertisements in local newspapers. Additional demographics, as well as physical activity levels, are available in Table 2. 


\section{Attrition Analyses}

Of the 787 participants who enrolled in the study, $321(40.8 \%)$ did not complete the first assessment, another 148 (18.8\%) were lost to follow up (i.e., they did not respond to the invitation for the three-month follow-up), and another 19 dropped out during the follow-up measurement, resulting in 299 (38.0\%) participants who completed all measures. We conducted three logistic regression analyses to investigate whether condition or any of the pre-test questionnaire measures could predict drop-out during the first measurement, loss to follow-up, and drop-out during the three-month follow-up measurement. The independent variables were frame, gender, age, ethnicity, education (we created two dummy variables to be able to estimate the contribution of the three education groups), mode of recruitment (we created four dummy variables to be able to estimate the contribution of the five mode of recruitment groups), baseline physical activity, and baseline intention. Results of the logistic regression analyses showed that age was the only significant predictor of attrition, such that older participants were less likely to drop out during the first measurement $(\mathrm{OR}=.98$, Wald $=$ 9.920, $p<.01)$, were less likely to be lost to follow-up $(\mathrm{OR}=.98$, Wald $=$ $11.786, p=.001)$, and were less likely to drop out during the follow-up measurement $(\mathrm{OR}=.98$, Wald $=14.149, p<.001)$.

\section{Regression analyses}

We conducted linear regression analyses to investigate the effect of frame on positive affect, negative affect, information acceptance, attitude, intention, and physical activity at follow-up, controlling for baseline physical activity and intention. Results showed that the gain-framed communication elicited more positive affect than the loss-framed communication, $\beta=.13, t(515)=3.19, p<$ $.01, s r=.13$, and less negative affect, $\beta=-.20, t(515)=-4.79, p<.001, s r=-.20$, suggesting that the manipulation of frame had been successful. Furthermore, the gain-framed communication resulted in marginally higher ratings of information acceptance, although the effect size was small, $\beta=.08, t(473)=1.72, p=.09, s r$ $=.08$. There was no significant effect of frame on attitude, $\beta=.06, t(488)=1.37$, $p=.17, s r=.06$. However, the gain-framed communication did result in stronger intentions to be physically active than the loss-framed communication, $\beta=.09$, $t(507)=2.66, p<.01, s r=.09$, although the semi-partial correlation revealed a small effect size. The gain-framed communication resulted in marginally greater levels of physical activity after three months, $\beta=.09, t(283)=1.70, p=.09, s r=$ .09 . 


\section{Discussion}

The present study investigated whether gain-framed messages were more persuasive than loss-framed messages advocating physical activity. To our knowledge, this is the first study to investigate the effects of framed messages in the context of a tailored intervention to promote physical activity. Our results suggest that framing does not have large and profound effects on relevant outcome measures. Although the means suggested that the gain-framed message received higher information acceptance scores, resulted in more positive attitudes, stronger intentions, and higher levels of physical activity at a three-month follow-up, only the effect of frame on intention was significant and all effects had small effect sizes, as indicated by semi-partial correlations of up to $s r=.10$.

These results, and the results of previous studies (Jones et al., 2003; 2004; McCall \& Ginis, 2004; Latimer et al., 2008a; 2008b) suggest that gainframed information promoting physical activity might be more persuasive than loss-framed information, but they also suggest that any advantage of gain- over loss-framed information is likely to be small. Thus, health-promotion practitioners who aim to promote physical activity might still be advised to emphasize the positive consequences of healthy behaviour instead of the negative consequences of unhealthy behaviour, if only for a total lack of evidence to suggest that loss-framed communications are more effective than gain-framed communications in promoting physical activity. It is also important to realize, however, that framing is hardly a 'magic bullet' that is likely to dramatically increase the effectiveness of health education interventions. In this regard, this study and the other studies on message framing and physical activity might be exemplary for the message-framing literature as a whole. Besides the occasional null-findings that have been reported (Brug, Ruiter, Van Assema, 2003; Jones et al., 2004; Steffen, Sternberg, Teegarden, \& Shepherd, 1994), recent meta-analyses provide evidence that gain- and loss-framed messages do not affect psychological and behavioural outcomes in a consistent and predictable way across all populations (O’Keefe \& Jensen, 2006; 2007).

To explore the reasons for the inconsistent results in the messageframing literature, research is needed that investigates under which circumstances gain- or loss-framed information is more persuasive. Research investigating possible moderating variables that can explain differences in the effects of gain- and loss-framed messages is indispensable to deepening our understanding of message framing's effects on persuasion. Previous research, for instance, has identified several potentially promising moderating variables, such as regulatory focus (Cesario, Grant, \& Higgins, 2004) and self-efficacy (Van 't Riet et al., 2008). Future research could further pursue this line of thought to be able to show us when gain-framed messages promoting physical activity are more effective and when loss-framed messages are more effective. 
24 Chapter 2

Table 2 Demographic profile of participants

\begin{tabular}{|c|c|}
\hline Variable & Percentage \\
\hline \multicolumn{2}{|l|}{ Sex } \\
\hline Male & 44.9 \\
\hline Female & 55.1 \\
\hline \multicolumn{2}{|l|}{ Ethnicity } \\
\hline Native & 87.0 \\
\hline Non-native & 13.0 \\
\hline \multicolumn{2}{|l|}{ Education } \\
\hline Low & 15.4 \\
\hline Medium & 41.9 \\
\hline High & 42.7 \\
\hline \multicolumn{2}{|l|}{ Recruitment } \\
\hline A search engine & 3.7 \\
\hline Hyperlink on related website & 25.8 \\
\hline Advertisement in newspaper & 19.2 \\
\hline Through family, friends, co-workers & 11.7 \\
\hline Local television & 39.6 \\
\hline \multicolumn{2}{|l|}{ Physical activity (minutes/day) } \\
\hline $0-15$ & 24.7 \\
\hline $16-30$ & 17.9 \\
\hline $31-45$ & 13.1 \\
\hline $46-60$ & 9.4 \\
\hline 61 or higher & 34.9 \\
\hline \multicolumn{2}{|l|}{ Intention } \\
\hline Intending to be physically active & 74.8 \\
\hline Not sure & 25.1 \\
\hline Not intending to be physically active & 12.2 \\
\hline
\end{tabular}

The present study contributes to the literature by being the first study to investigate the effects of framed information in the context of a computertailored intervention. Computer tailoring offers a useful tool for health education practice and is widely used (Noar et al., 2007). The results of the present study thus supports the finding that message framing does not have large effects on relevant outcome measures (O'Keefe \& Jensen, 2007), but offers the additional advantage of increased external validity. The results suggest that, as yet, other tools to increase the impact of health education interventions might be more effective than message framing. In fact, the results of studies on computer tailoring suggest that computer tailoring itself can be an effective means to promote a healthy lifestyle (Kreuter et al., 2000; Noar et al., 2007). For the moment, it thus seems that computer tailoring offers a more promising approach to healthpromoting interventions than message framing. 
The present study was subject to certain limitations. First, our recruitment method resulted in a sample that was highly educated as compared with the general Dutch population. Whereas only $25.1 \%$ of the Dutch working population has a high education level (CBS, 2006), in our sample, $42.7 \%$ was highly educated. Second, our sample was highly physically active before the start of the study. Whereas national data suggests that only half of the Dutch population is sufficiently physically active (Wendel-Vos, 2008), 57.4\% of our sample seemed to meet the recommendations with regards to physical activity. Because many health-education efforts are primarily targeted at those who do not engage in healthy behaviour, future research should investigate the effects of tailored feedback and framed information in people who are insufficiently physically active. A third limitation of the present study is the high attrition rate. Even though participants indicated to be highly motivated to engage in physical activity, large numbers of them quickly lost interest in the study. Although attrition rates this high are not uncommon in on-line research (Etter, 2006; Eysenbach, 2005; Tate \& Zabinski, 2004), future studies should try to minimize drop-out to be able to generalize the results. Finally, the fact that we did not include a condition in which participants received non-tailored information before they received the framed information made it impossible to compare the effects of framing in the context of a tailored versus a non-tailored intervention.

A strength of our study is the fact that we included a three-month follow-up. Many previous message framing studies have used immediate post-tests only and have relied on attitudes or intentions as the main outcome measures. Our three-month follow-up made it possible to assess the effects of the framed messages on behaviour.

\section{Conclusion}

On the basis of this study and previous studies investigating the effects of framed information advocating physical activity, it is hard to arrive at definitive recommendations for health-promotion practitioners. Given that, in the domain of physical activity, some studies (including the present study) show a small advantage of gain-framed information, and no study to our knowledge reported an advantage of loss-framed information, we suggest that information promoting physical activity is best framed in terms of gains. It seems, however, that the effects of framing are likely to be small. 

Part 2

Underlying Mechanisms 



\title{
Chapter 3: Distinct Pathways to Persuasion: The Role of Affect in Message-Framing Effects ${ }^{5}$
}

\begin{abstract}
Health-promoting messages can be framed in terms of the gains that are associated with healthy behaviour (gain-framed) or the losses that are associated with unhealthy behaviour (loss-framed). In the present research, we examined the role of positive and negative affect in the persuasive effects of gain- and loss-framed health promoting information. Study $3.1(\mathrm{~N}=100)$ showed that gain-framed information resulted in higher levels of information acceptance than loss-framed information and this effect was mediated by positive affect. The results of Study $3.2(\mathrm{~N}=129)$ showed that gain-framed information resulted in higher levels of information acceptance and attitude, an effect that was again mediated by positive affect. In addition, loss-framed information resulted in more negative affect than gain-framed information and negative affect increased participants' intention to engage in the healthy behaviour. These results suggest that affect may be of great importance in the persuasion process and may be particularly helpful to explain the underlying mechanisms of message framing effects. The findings also suggest that gain- and loss-framed messages offer distinct pathways to persuasion.
\end{abstract}

\section{Introduction}

Health promoting messages usually stress the value of healthy behaviour, but they can do this in several ways. In particular, the message can be framed in terms of the benefits of engaging in the behaviour (gain frame) or in terms of the costs of failing to engage in the behaviour (loss frame). Research has shown that these frames can have a different persuasive impact, even when the different frames are applied to objectively equivalent information (see for instance Gerend \& Cullen, 2008; O'Keefe \& Jensen, 2006; Rothman \& Salovey, 1997; Van 't Riet, Ruiter, Werrij, \& De Vries, 2008).

Unfortunately, studies show inconsistent results with regard to which type of framing is more persuasive (O'Keefe \& Jensen, 2006). To account for the inconsistent findings in the literature, one influential approach to the study of message framing has been to focus on the function of the recommended behaviour, making a distinction between behaviours that serve to prevent an illness (like exercising or quitting smoking) and behaviours that serve to detect an illness (like skin self-examination or obtaining a mammography). According to Rothman and Salovey (1997), people perceive disease-prevention behaviours as relatively safe, because they minimize the chance of falling ill. In contrast, peo- 
ple perceive disease-detection behaviours as inherently risky because they entail the possibility of finding out that one is ill. Drawing from Prospect Theory (Kahneman, 2003; Kahneman \& Tversky, 1984), Rothman and Salovey (1997) go on to suggest that gain-framed information is more persuasive when advocating disease-prevention behaviours, because gain-framed information makes people risk-averse and thus more likely to engage in relatively safe disease-prevention behaviours. In contrast, they suggest that loss-framed information is more persuasive for disease-detection behaviours, because loss-framed information makes people willing to take risks and thus more likely to engage in relatively risky disease-detection behaviours. A recent meta-analysis showed, however, that for behaviours serving to detect an illness, gain- and loss-framed messages were not differentially persuasive (O'Keefe \& Jensen, 2006). For behaviours serving to prevent an illness, a small advantage of gain-framed information was found, although this effect seemed mainly due to a limited amount of studies on dental health (O'Keefe \& Jensen, 2007). Thus, in contrast to Rothman and Salovey's reasoning, and despite an impressive body of literature, it is currently unclear how health-care professionals should frame persuasive messages.

In the present research, we tried to shed some light on the inconsistent findings in the message-framing literature, by investigating the underlying mechanisms of framing effects. Because finding no differential effects of message framing might obscure the fact that different but equally potent mechanisms might underlie the effects of gain- and loss-framed information (Shen \& Dillard, 2007), we tried to investigate the pathways through which gain- and loss-framed information exert their influence on persuasion. Research has shown that gainframed information evokes positive feelings in the recipient and that loss-framed information evokes negative feelings (e.g., Shen \& Dillard, 2007). In the present paper, we propose that positive and negative affect can both contribute to persuasion.

\section{The Role of Affect}

Contemporary theories of emotion state that affect results from an appraisal of the relationship between one's goals and the environment. This appraisal yields positive affect when an individual perceives that the environment is congruent with his or her goals. In contrast, when an individual judges that the environment is in an incongruent relationship with his or her personal goals, the individual experiences negative affect (for overviews see Frijda, 1986; Lazarus, 1991). The purpose of affect is to induce states of 'action readiness' that make the individual responsive to the environment or context (Frijda, 2007). As Shen and Dillard (2007) point out, gain-framed health-promoting messages focus on beneficial outcomes, emphasizing possible congruence between the recipient's goals and the environment/situation. Loss-framed health promoting messages, on the other hand, focus on costs and by doing so emphasize possible incongruence 
between goals and the environment/situation. For this reason, we expect gainframed information to elicit more positive affect than loss-framed information and loss-framed information to elicit more negative affect than gain-framed information. The limited research that exists on the relationship between framing and affect is consistent with our logic (Chang, 2005; Cox, Cox, \& Zimet, 2006; Cox \& Cox, 2001; Millar \& Millar, 2000; Schneider et al., 2001a; Shen \& Dillard, 2007).

The idea that affect plays a crucial role in persuasion has a long history in Western thought. According to Aristotle, there are three means of persuasion: the use of logic, the moral character of the speaker and the elicitation of emotion in the listener (Carey, 1994). In recent years, several scholars have studied the effects of message-induced affect on persuasion. One theoretical account of the relationship between message-induced affect and persuasion stems from marketing and consumer psychology and is called the 'affect transfer hypothesis' (MacKenzie, Lutz, \& Belch, 1986). According to this account, advertisements that evoke positive affect result in a positive attitude towards the ad, which in turn results in a positive attitude towards the advertised brand. A meta-analysis of persuasion studies in the field of marketing showed support for this hypothesis (Brown, Homer, \& Inman, 1998).

Negative affect has also drawn attention from persuasion scholars. Numerous studies tested the effects of fear appeals on measures of attitude, intention and behaviour (for overviews see Eagly \& Chaiken, 1993; Ruiter, Abraham, $\&$ Kok, 2001). A fear appeal is a persuasive message attempting to arouse fear to promote precautionary motivation and self-protective action (Rogers, 1983). Meta-analyses of the fear-persuasion relationship have shown that evoking fear can enhance a message's persuasive power (Mongeau, 1998; Witte \& Allen, 2000; but see also Ruiter et al., 2001). With regards to framed messages, the persuasive effects of positive and negative affect imply that gain- and loss-framed messages exert their influence on persuasion to a large extent through quite different substrates (positive versus negative affect; see also Cacioppo \& Berntson, 1994).

\section{Mood and Persuasion}

There is an extensive body of literature available on the influence of affect on information processing and decision making and several theoretical frameworks have been proposed to account for the empirical findings (e.g., Bless \& Schwartz, 1999; Forgas, 1995; Isen, Daubman, \& Nowicki, 1987; Isen, Nygren, \& Ashby, 1988). Most of this research, however, has focused on the effects of moods. In a typical experiment, mood is induced by having participants watch either a happy or sad videotape (e.g., Forgas, 2007) or a positive mood is induced by giving participants a small bag of candies (e.g., Isen et al., 1987), after which happy and sad participants (or happy participants and participants in a 
neutral mood) engage in the experimental task. There is reason to believe that mood is particularly likely to exert effects on people's thoughts and behaviour when individuals are unaware of the source of their affect (Sinclair, Mark, \& Clore, 1994). According to Schwarz and Clore (2003; p. 299-300): 'What renders the influence of moods highly pervasive is that their source is usually not in the focus of our attention.' In the present research, we will not focus on moods but on what Dillard and Meijnders (2002) call 'message-induced affect', affect that is a product of the message itself and that is most likely to be consciously perceived by recipients as arising from processing the persuasive message. Dillard and Meijnders state that message-induced affect differs from 'messageirrelevant affect', such as moods, which bears no logical relationship to the content of the message and is mainly influential when people are not aware of its source.

\section{The Present Research}

We conducted two experiments to test whether framing exerts its effects on persuasion through positive and negative affect. In Study 3.1, we used framed information about physical activity, assessing acceptance of the information as the outcome measure. In Study 3.2, we used framed information about reducing salt intake, assessing information acceptance, attitude, intention and behaviour as the outcome measures. Our hypotheses were that gain-framed information would result in more positive affect than loss-framed information (Hypothesis 1) and that positive affect would mediate the persuasiveness of gain-framed information (Hypothesis 2). In addition we hypothesized that loss-framed information would result in more negative affect than gain-framed information (Hypothesis 3 ) and that negative affect would mediate the persuasiveness of loss-framed information (Hypothesis 4).

\section{Study 3.1}

\section{Method}

Participants. One hundred students at Maastricht University voluntarily participated in the experiment. Most of the participants were female (77 versus 22 males; one participant failed to specify gender). Age ranged from 18 to 36 years, with a mean age of 20.8 years $(\mathrm{SD}=2.4)$.

Procedure and design. Participants were recruited in the university restaurant buildings. Students who were sitting at one of the restaurant tables during lunchtime were eligible to participate. Participants were asked to fill in a questionnaire containing questions on physical activity. These students received a booklet containing a written introduction of the experiment and the questionnaire, which, following previous studies in which framing effects were found as 
a result of framed information that consisted of no more than a few statements (Broemer, 2004), consisted of three pages in which three statements concerning physical activity were used as the framed material. The three pages each started with a statement, followed by seven questions that assessed the extent to which the statement elicited positive and negative affect and participants' acceptance of the statement. In the gain-framed information condition all three statements were gain-framed, whereas in the loss-framed information condition, all three statements were loss-framed. To avoid sequence-effects the order of the three statements was varied randomly. The experiment used a one-factorial (frame: gain vs. loss) between-participants design.

Framed materials. The three gain-framed [loss-framed] statements on physical activity were 'If I am [in]sufficiently active, my muscles will stay strong [become weak]', 'If I am [in]sufficiently active, my immunity will increase [suffer], so it will be easier to stay healthy [harder to fight of diseases]' and 'If I am [in]sufficiently active, I will have a smaller chance [greater risk] of getting cardiovascular disease'. Pilot studies showed that these statements were judged as credible.

Outcome measures. For each statement, two items assessed positive affective reactions to the statement and two items assessed negative affective reactions, using 10-point semantic differentials. The positive affect items assessed the extent to which participants thought the statement made them feel happy $(1=$ Very happy; $10=$ Not happy at all $)$ and made them feel relieved $(1=$ Very relieved; $10=$ Not relieved at all). Scores on these items were reversed such that a high score reflected a positive subjective feeling and then combined to create an average positive affect score. The internal consistencies of these scales were calculated for all three statements separately and ranged from Cronbach's $\alpha=.64$ to $\alpha=.84$. The average internal consistency over all three statements was $\alpha=.75$.

The negative affect items assessed the extent to which participants thought the statement made them feel sad $(1=$ Very sad; $10=$ Not sad at all $)$ and afraid $(1=$ Very afraid; $10=$ Not afraid at all $)$. Scores on the sad and afraid items were reversed and combined to create an average negative affect score. The average internal consistency over all three statements was $\alpha=.72$ (range: $\alpha$ $=.66$ to $\alpha=.77$ ). Correlations between positive and negative affect ranged from $r=-.23$ to $r=.05$. The overall correlation between the two affect variables was $r$ $=-.08$.

Three items assessed information acceptance by asking participants to indicate for each statement the extent to which they agreed with the statement (1 = I totally agree $; 10=$ I totally disagree $)$, felt that the statement was exaggerated $(1=$ Very exaggerated $; 10=$ Not at all exaggerated $)$, and thought the statement was relevant $(1=$ Very relevant; $10=$ Not at all relevant $)$. After we reversed the scores on the agreement and relevance items, the scores on the three items were averaged to create an average information acceptance score for each statement. 
The average internal consistency over all three statements was $\alpha=.77$ (range: $\alpha$ $=.72$ to $\alpha=.83$ ).

Statistical analysis. First, correlations between positive affect, negative affect and information acceptance were investigated. Second, the effect of frame on affect and information acceptance was investigated using linear multilevel analyses to account for the fact that the measures of negative and positive affect were clustered within individuals. In these analyses, frame was coded as $0=$ gain frame, 1 = loss frame. In addition, two dummy variables indicating the statement were generated and entered into all linear multilevel analyses (dummy 1 was coded as $0=$ statement $1 /$ statement $3,1=$ statement 2 ; dummy 2 was coded as 0 = statement $1 /$ statement $2,1=$ statement 3 ). Since they are not of central concern for the present research, results for these dummy variables will not be reported in this paper. We used the module Mixed Linear Models within the software package SPSS 13.0.

\section{Results}

Correlations. As can be seen in Table 3.1, information acceptance was positively correlated with positive affect but not with negative affect. Positive and negative affect were not significantly correlated.

Results of the linear multilevel analyses. Gain-framed statements elicited more positive affect than loss-framed statements, supporting Hypothesis 1 $\left(\mathrm{M}_{\text {gain }}=5.61 ; \mathrm{M}_{\text {loss }}=5.01\right)$. Frame did not have a significant effect on negative affect $\left(\mathrm{M}_{\text {gain }}=3.17 ; \mathrm{M}_{\text {loss }}=2.90\right)$, but did have a marginally significant effect on information acceptance, such that gain-framed statements received higher ratings of information acceptance than loss-framed statements $\left(\mathrm{M}_{\text {gain }}=7.44 ; \mathrm{M}_{\text {loss }}=\right.$ 6.87; see Table 3.2).

Mediation analyses. Mediation analyses were performed to investigate whether the marginally significant effect of frame on information acceptance was mediated by positive affect. For this purpose, we conducted a test of the joint significance of the two effects comprising the mediation effect (MacKinnon, Lockwood, Hoffman, West, \& Sheets, 2002). In this approach a variable functions as a mediator when two conditions are met: (a) the independent variable has a significant effect on the mediator (Path A), and (b) the mediator is significantly associated with the dependent variable when the other independent variables are controlled for (Path B). In the commonly used Baron and Kenny approach (Baron \& Kenny, 1986) two other conditions are posited: (1) the independent variable has a total effect on the dependent variable and (2) this total effect is reduced when the mediator is entered into the model. However, for the present research, the approach as outlined by MacKinnon and colleagues (2002) has two advantages over the traditional Baron and Kenny approach. Firstly, the test of joint significance has been shown to have greater power (MacKinnon et al., 2002). Secondly, since we hypothesize that framing exerts a different influ- 
ence on positive and negative affect (gain-framed information results in more positive affect than loss-framed information, but in less negative affect) and positive and negative affect exert the same influence on information acceptance we do not assume the existence of a total effect of framing on information acceptance. Table 3.2 shows that frame had a significant effect on positive affect (Path A). To test whether positive affect had a significant effect on information acceptance while frame was controlled for (Path B) another mixed linear model analysis was conducted with frame and positive affect as independent variables and information acceptance as the dependent variable. In this analysis, positive affect had a significant effect on information acceptance, $B=.32, t=4.58, p<.001$. This supports Hypothesis 2 and suggests that the effect of frame on information acceptance was mediated by positive affect. A Sobel's Z test (i.e., the Aroian version of the $Z$ test; Baron \& Kenny, 1986) revealed that the mediation was marginally significant, $\mathrm{Z}=1.85, p=.06$.

Table 3.1 Correlations (range of correlations computed for the three statements separately) between positive affect, negative affect and information acceptance

\begin{tabular}{lll}
\hline & Positive affect & Negative affect \\
\hline Negative affect & $-.08(-.23-.05)$ & - \\
Information acceptance & $.30 *(.28-.34)$ & $.00(-.10-.13)$ \\
\hline & & \\
$* \mathrm{p}<.05$ & & \\
$* * \mathrm{p}<.01$ & &
\end{tabular}

Table 3.2 Results of the linear multilevel analyses: Effects of frame on positive affect, negative affect and information acceptance

\begin{tabular}{lll}
\hline & \multicolumn{2}{l}{ Effect of Frame $^{\mathrm{a}}$} \\
& $\mathrm{B}$ & $\mathrm{SE} \mathrm{B}$ \\
\hline Positive affect & $-.59^{*}$ & .29 \\
Negative affect & -.27 & .33 \\
Information acceptance & $-.58^{+}$ & .31 \\
\hline & & \\
& & \\
${ }^{\mathrm{a}}$ coded as $0=$ gain frame, 1 = loss frame & \\
${ }^{+} \mathrm{p}<.10$ & & \\
$* \mathrm{p}<.05$ &
\end{tabular}

Discussion and Introduction to Study 3.2.

The results of Study 3.1 showed that individuals found gain-framed information about physical activity marginally more convincing than loss-framed information and that positive affect mediated this effect, supporting Hypothesis 1 
and 2 and suggesting that positive affect can be of great importance in the persuasion process. We did not find evidence that loss-framed information evoked higher levels of negative affect than gain-framed information or that negative affect had an effect on persuasion. However, this failure to find support for Hypotheses 3 and 4 might be caused by the limitations of Study 3.1. For one, the fact that we did not find an effect of frame on negative affect might be due to the format of the information (i.e., short, written statements). Three single statements might have been too weak to produce noticeable differences in negative affect, especially because university students are not likely to worry greatly about distal threats such as cardiovascular disease, which is illustrated by the low means of the negative affect scores $\left(\mathrm{M}_{\text {gain }}=3.17 ; \mathrm{M}_{\text {loss }}=2.90\right.$ on a 10-point scale $)$. For this reason Study 3.2 made use of health-promoting messages consisting of up to 190 words and containing a number of arguments to make sure that the framed information was substantial enough to be able to evoke negative affect. A second limitation of Study 3.1 was that the research setting (the university restaurant during lunchtime) limited experimental control and although we instructed participants to fill out the questionnaires individually we can not be sure that all participants refrained from communicating with each other during the procedure. For this reason, we conducted Study 3.2 in our research lab, increasing experimental control. Third, although perceived effectiveness, a measure akin to information acceptance, has been shown to be substantially correlated with actual effectiveness (Dillard, Weber, \& Vail, 2007), finding effects of framing on information acceptance is less relevant than, for instance, finding effects on attitude, intention or behaviour. Moreover, it is possible that positive and negative affect have different effects on different outcome measures.

According to Fredrickson and Losada (2005), positive and negative emotions both have adaptive value, but in a different way. Positive emotions can broaden people's mindsets, expanding cognition and behavioural tendencies (Fredrickson \& Branigan, 2005). This may account for the fact that participants in the gain-framed condition were more 'open' to the health-promoting information. One might also hypothesize that this openness leads to a more positive attitude towards the recommended behaviour, which is supported by findings from the marketing literature that suggest that positive affect can contribute to a positive attitude towards the advertised brand (Brown et al., 1998). Negative emotions, on the other hand, narrow people's behavioural urges toward specific actions, preparing the body physically and mentally for immediate action (Fredrickson and Losada, 2005; see also Frijda's (2007) conceptualization of action readiness). Thus, negative affect as a consequence of loss-framed information, might exert a motivating influence more directly on intention. Some evidence for this comes from studies in which participants' self-reported negative affect after reading a health-promoting message was significantly correlated with 
intention, but not with attitude (De Hoog, Stroebe, \& De Wit, 2008, Experiment 2; Witte, 1994).

To obtain a clearer picture of the role of positive and negative affect in message-framing effects, we used in Study 3.2 gain- and loss-framed messages about reducing salt intake and assessed positive affect, negative affect, information acceptance, attitude, intention, and behaviour. Our hypotheses for Study 3.2 were again that gain-framed information would result in more positive affect than loss-framed information (Hypothesis 1) and that positive affect would mediate the effect of gain-framed information on information acceptance and attitude (Hypothesis 2). In addition we hypothesized that loss-framed information would result in more negative affect than gain-framed information (Hypothesis 3 ) and that negative affect would mediate the effect of loss-framed information on intention (Hypothesis 4).

\section{Study 3.2}

\section{Method}

Participants. One hundred and twenty-nine students at Maastricht University voluntarily participated in the experiment in exchange for $€ 10$. Most of the participants were female (114 versus 15 males). Age ranged from 17 to 25 years, with a mean age of 20.1 years $(\mathrm{SD}=1.7)$.

Procedure and design. Participants were seated in individual booths and were told that they were about to participate in a study aimed at testing health education materials that had been designed to be used 'on the Internet'. First, participants were provided with a persuasive communication about reducing salt intake, which was either gain-framed or loss-framed. After reading the persuasive communication, participants completed the dependent measures. One week after this, they returned to the laboratory to assess whether they had reduced their salt intake. One participant failed to keep his appointment for the second measurement and, as a consequence, no assessment of behaviour could take place. After the second measurement, participants were debriefed and received their fee. This study used a one-factorial (frame: gain vs. loss) between participants design.

Framed communication. The persuasive communication consisted of a short message about either the positive consequences of reducing the amount of salt in one's diet or the negative consequences of not reducing the amount of salt in one's diet. The gain-framed message contained 195 words and the loss-framed message contained 180 words. The full texts are available in Appendix B.

Outcome measures. Four items assessed positive reactions to the information (positive affect) and four items assessed negative reactions (negative affect). The positive affect items assessed the extent to which participants thought 
the information made them feel happy $(1=$ Very happy; $7=$ Not at all happy $)$, relieved $(1=$ Very relieved; $7=$ Not at all relieved $)$, optimistic $(1=$ Very optimistic $; 7=$ Not at all optimistic $)$, and content $(1=$ Very content $; 7=$ Not at all content). Scores were reversed and combined to create an average positive affect score, $\alpha=.84$. The negative affect items assessed the extent to which participants thought the information made them feel sad $(1=$ Very sad; $7=$ Not at all sad $)$, afraid $(1=$ Very afraid $; 7=$ Not at all afraid $)$, tense $(1=$ Very tense $; 7=$ Not at all tense $)$, and uncomfortable ( $1=$ Very uncomfortable; $7=$ Not at all uncomfortable). Scores were reversed and combined to create an average negative affect score, $\alpha=.86$.

Eight items assessed information acceptance by asking participants to indicate the extent to which they thought the information was convincing ( $1=$ Very convincing; $7=$ Not at all convincing $)$, relevant $(1=$ Very relevant $;=$ Not at all relevant $)$, realistic $(1=$ Very realistic $; 7=$ Not at all realistic $)$, useful $(1=$ Very useful; $7=$ Not at all useful $)$, interesting $(1=$ Very interesting; $7=$ Not at all interesting $)$, true $(1=$ Very true; $7=$ Not at all true $)$ and exaggerated $(1=$ Very exaggerated; $7=$ Not at all exaggerated). Furthermore, one item asked participants to indicate the extent to which participants agreed with the information (1 $=$ I totally agree; $7=$ I totally disagree). After we reversed the scores of all items except the exaggerated item, the scores on the eight items were averaged to create an average information acceptance score, $\alpha=.78$.

Six items were used to assess attitude towards eating less salt, asking participants to indicate the extent to which they rated eating less salt as (1) very good vs. (7) very bad, (1) very healthy vs. (7) very unhealthy, (1) very tasty vs. (7) not at all tasty, (1) very much worth the effort vs. (7) not worth the effort at all, (1) very important vs. (7) very unimportant, and (1) very sensible vs. (7) not sensible at all. After scores on the attitude items were reversed, an average score was created, $\alpha=.81$.

Six items were used to assess intention to eat less salt. One item asked participants 'how likely is it that you will eat less salt in the future?' $(1=$ Very likely; 7 = Very unlikely). One item asked participants: 'how likely is it that you will eat less salt in the coming week?' ( 1 = Very likely; 7 = Very unlikely $)$. Two items asked participants whether they planned to eat less salt in the future and in the coming week. One item asked participants whether they considered eating less salt in the future and one item asked participants whether they were sure that the would eat less salt in the coming week. These four items used a 7-point scale $(1=$ Certainly; $7=$ Certainly not $)$. Scores on the intention items were reversed and an average intention score was calculated, $\alpha=.94$.

One item assessed behaviour. When participants returned to the laboratory one week after reading the framed communication, one item asked them whether they thought they had eaten less salt in the last 7 days, as compared with before participating in the experiment $(1=$ certainly not; $7=$ certainly $){ }^{6}$ 
Statistical analysis. First, correlations between all relevant variables were investigated. Second, linear regression analyses tested the effect of frame (coded as $0=$ gain frame, $1=$ loss frame) on positive affect, negative affect, information acceptance, attitude, intention, and behaviour. Third, mediation analyses were performed. The used statistical package was SPSS 13.0. Fourth, relations between relevant variables were investigated using Structural Equation Modeling. For this the statistical package M-Plus version 3.11 was used.

\section{Results}

Correlations. Positive and negative affect had a significant, negative correlation. Furthermore, positive affect was significantly correlated with information acceptance and attitude, whereas negative affect was significantly correlated with intention (see Table 3.3).

Regression analyses. Results of the linear regression analyses showed that gain-framed messages evoked more positive affect than loss-framed messages $\left(\mathrm{M}_{\text {gain }}=4.63 ; \mathrm{M}_{\text {loss }}=4.23\right)$ whereas loss-framed messages evoked more negative affect $\left(\mathrm{M}_{\text {gain }}=3.12 ; \mathrm{M}_{\text {loss }}=3.46\right)$, supporting hypotheses 1 and 3. Also, gain-framed information was more readily accepted than loss-framed information, as in Study $3.1\left(\mathrm{M}_{\text {gain }}=5.71 ; \mathrm{M}_{\text {loss }}=5.47\right)$, and resulted in more positive attitudes $\left(\mathrm{M}_{\text {gain }}=5.53 ; \mathrm{M}_{\text {loss }}=5.32\right)$, although the latter difference was only marginally significant, $p=.08$.

Mediation analyses. Mediation analyses were performed to investigate whether the effect of frame on information acceptance and the marginally significant effect of frame on attitude were mediated by positive affect. Also, we investigated whether negative affect mediated an effect of frame on intention. For this purpose, we conducted a test of the joint significance of the two effects comprising the mediation effect (MacKinnon et al., 2002; see Study 3.1).

Table 3.4 shows that frame had a significant effect on positive affect (Path A). To test whether positive affect had a significant effect on information acceptance and attitude while frame was controlled for (Path B), we conducted two regression analyses with frame and positive affect as independent variables and information acceptance and attitude as the dependent variable. In the first analysis, positive affect had a significant effect on information acceptance, $B=$ $.22, t(125)=3.73, p=.001$. In the second analysis, positive affect had a significant effect on attitude, $B=.37, t(125)=5.59, p<.001$. This suggests that the effect of frame on information acceptance and the effect of frame on attitude were both mediated by positive affect. Two Sobel's $Z$ tests revealed that the mediation was significant for information acceptance, $Z=2.04, p<.05$, and for attitude, $\mathrm{Z}=2.42, p<.05$. Table 3.4 also shows that frame had a significant effect on negative affect. A third regression analysis was performed to investigate whether negative affect had a significant effect on intention when controlled for 
frame. This proved to be the case, $B=.37, t(125)=8.58, p<.001$. A Sobel's $Z$ tests revealed that the mediation was significant, $\mathrm{Z}=1.92, p=.05$.

Table 3.3 Correlations between positive affect, negative affect, attitude, intention and behaviour

\begin{tabular}{llllll}
\hline & $\begin{array}{l}\text { Positive } \\
\text { Affect }\end{array}$ & $\begin{array}{l}\text { Negative } \\
\text { Affect }\end{array}$ & $\begin{array}{l}\text { Information } \\
\text { Acceptance }\end{array}$ & Attitude & Intention \\
\hline Negative Affect & $-.31^{* *}$ & - & & \\
Information Acceptance & $.31^{* *}$ & -.12 & - & & \\
Attitude & $.47^{* *}$ & -.17 & $.58^{* * *}$ & - & \\
Intention & .02 & $.27^{* *}$ & $.20^{*}$ & $.35^{* * *}$ & - \\
Behaviour & .10 & -.02 & .06 & $.21^{*}$ & $.46^{* * *}$ \\
\hline
\end{tabular}

$* \mathrm{p}<.05$

$* * \mathrm{p}<.01$

$* * * \mathrm{p}<.001$

Table 3.4 Results of the linear regression analyses: Effects of frame on positive affect, negative affect, information acceptance, attitude, intention and behaviour

\begin{tabular}{lll}
\hline & \multicolumn{2}{l}{ Effect of Frame $^{\mathrm{a}}$} \\
& $\mathrm{B}$ & $\mathrm{SE} \mathrm{B}$ \\
\hline Positive affect & $-.39^{* *}$ & .14 \\
Negative affect & $.34^{*}$ & .17 \\
Information acceptance & $-.25^{*}$ & .11 \\
Attitude & $-.21^{+}$ & .12 \\
Intention & -.05 & .22 \\
Behaviour & .12 & .25 \\
\hline & & \\
${ }^{\mathrm{a}}$ coded as $0=$ gain frame, 1 & \\
$+\mathrm{p}<.10$ & & \\
$* \mathrm{p}<.05$ & \\
$* * \mathrm{p}<.01$ &
\end{tabular}

Path Model. In addition to the mediation analyses presented above, we used Structural Equation Modeling to get a clearer picture of our results. To investigate the influence that positive and negative affect had on the other outcome measures, we investigated several path models. Figure 3 shows the path model with the most favorable model fit, in which message frame predicts both positive and negative affect, positive affect predicts information acceptance and attitude while negative affect has a direct effect on intention. Analyses using Structural 
Equation Modeling showed that this model fitted the data well, $\chi^{2}(12)=8.430, p$ $=.7507 ; \mathrm{CFI}=1.000 ; \mathrm{RMSEA}=.000 ; \mathrm{SRMR}=.040 .^{7}$ To obtain an additional test of our model, an extended model was tested in which paths from frame to positive affect, negative affect, information acceptance, attitude, intention, and behaviour were added, as were paths from positive and negative affect to information acceptance, attitude, intention, and behaviour. This extended model did not have a significantly greater model fit than the model in Figure $3, \Delta \chi^{2}(9)=$ 7.426, $p=$ n.s. The paths from frame to information acceptance, attitude, intention, and behaviour were not significant, and neither were the paths from positive affect to intention and behaviour, or the paths from negative affect to information acceptance, attitude, and behaviour.

\section{Discussion}

The results of Study 3.2 confirmed Hypotheses 1 and 2 and replicated the finding from Study 3.1 that gain-framed information lead to greater levels of positive affect, which was positively associated with information acceptance. In addition, an effect of positive affect on attitude was found. In Study 3.2 we further found that loss-framed information resulted in greater levels of negative affect and that negative affect lead to stronger intentions to reduce salt intake, supporting Hypotheses 3 and 4.

We propose that these findings suggest that gain- and loss-framed messages not only exert their influence through separate mechanisms (positive and negative affect), but also that these mechanisms might have different effects on different outcome measures.

\section{General Discussion}

The aim of the present research was to investigate whether information that is gain- versus loss-framed gives rise to different levels of positive and negative feelings and whether these feelings exert an influence on persuasion. The results of two experiments showed that gain-framed information evoked greater levels of positive affect in recipients than loss-framed information, and that positive affect in turn was positively associated with information acceptance, resulting in greater levels of information acceptance in the gain-framed condition. In Study 3.2, positive affect also influenced participants' attitudes, resulting in marginally more positive attitudes in the gain-framed condition as compared to the loss-framed condition. The results of Study 3.2 furthermore showed that lossframed information resulted in greater levels of negative affect than gain-framed information and that negative affect, while not being associated with information acceptance or attitude, had a significant effect on intention to reduce salt intake. Whereas the mediation effect of positive affect was only marginally significant 
in Study 3.1, in Study 3.2 frame had a significant effect on positive and negative affect (Hypotheses 1 and 2) and the mediation analyses showed clear support for a mediating effect of positive affect on information acceptance and attitude (Hypothesis 3), and of negative affect on intention (Hypothesis 4). We did not find direct effects of either positive or negative affect on behaviour.

Figure 3 Path model showing positive affect as a predictor of information acceptance and attitude, and negative affect as a predictor of intention"

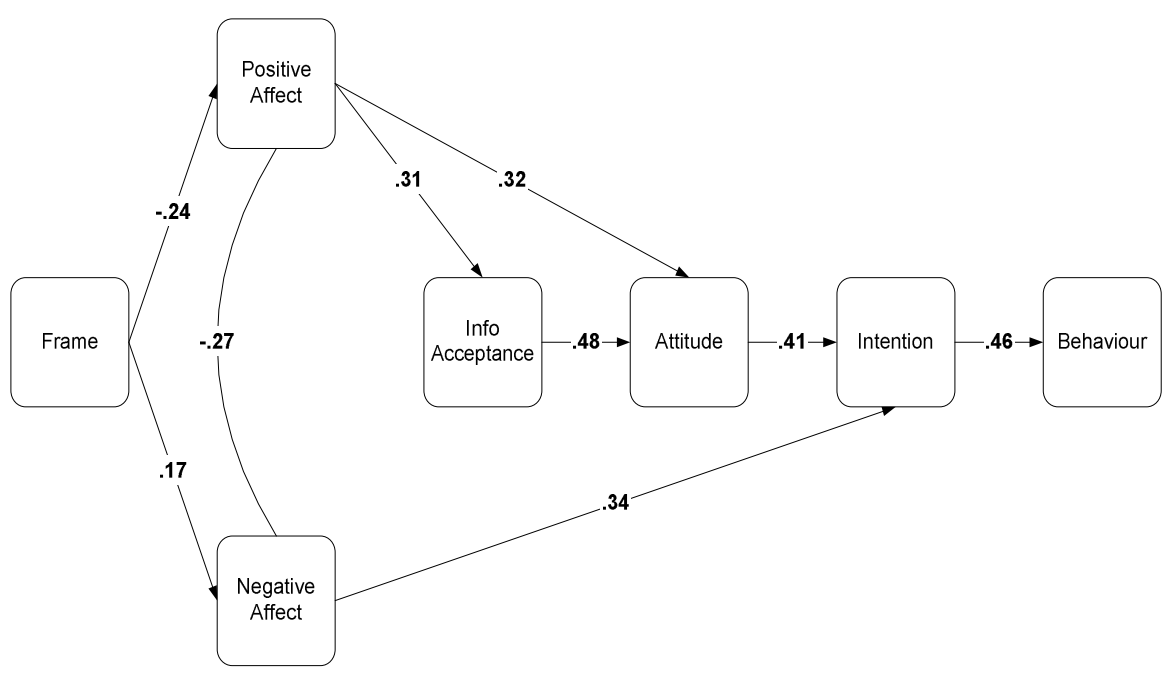

${ }^{*}$ All paths are significant at the $\mathrm{p}=.05$ level

These results suggest that positive and negative affective reactions are of great importance to the study of message framing in particular and persuasion in general. They suggest that gain- and loss-framed messages exert their influence through separate mechanisms (positive and negative affect). In fact, although more research is needed to arrive at definitive conclusions, it seems that gain-framed information can have an effect on information acceptance and attitude through positive affect, exerting a persuading influence, whereas lossframed information might be more likely to havi ${ }^{34}$ effect on intention through negative affect, exerting a motivating influence.

Our results also show that, since framing has opposing effects on positive and negative affect, and positive and negative affect both contribute to persuasion, it can be difficult to find consistent main effects of framing on behaviour. In this regard, our results might be exemplary of the message-framing literature as a whole. Besides the occasional null-findings that have been reported 
(Brug, Ruiter, \& Van Assema, 2003; Jones, Sinclair, \& Courneya, 2003), recent meta-analyses provide evidence that gain- and loss-framed messages do not affect psychological and behavioural outcomes in a consistent and predictable way across all populations (O'Keefe \& Jensen, 2006; 2007). Our findings add to the literature, however, by implying that message-framing research should pay specific attention the underlying mechanisms of framing effects and to the outcome measures being used. In a recent meta-analysis, for instance, O'Keefe and Jensen included dozens of studies, without differentiating between effects found on attitude, intention or behaviour. In fact, when multiple indices of persuasion were available, they averaged the effects, yielding a single summary. A meta-analysis of this kind, focusing on outcomes instead of processes and not differentiating between outcome measures, obscures the possibility that gain- and loss-framed messages may exert their influence on persuasion through different pathways (Shen \& Dillard, 2007). The results of the present research suggest that future meta-analyses should analyze the effects of framing on information acceptance, attitude, intention, and behaviour separately.

In addition, research that does not distinguish between outcome measures might be unable to reveal effects that are relevant to health-promotion professionals. For instance, the effect of frame on information acceptance, also found in previous studies (Latimer et al., 2008a; Reinhart, Marshall, Feeley, \& Tutzauer, 2007; Schneider et al., 2001b; Smith, 1996), might have direct implications for health-promoting materials on the Internet. Given the growing practice to use the Internet as a medium for health-promoting information and the importance of increasing people's exposure to this information (i.e., drawing attention and keeping recipients interested; see for instance Crutzen et al., 2008) information acceptance might be of critical importance. After all, people seeking information on the Internet are most likely to select information that seems relevant, interesting, and trustworthy. Future research should investigate whether message framing can be used to increase exposure in an online setting.

In the present article, we have argued that, to better understand message-framing effects, it is important to investigate the pathways by which gainand loss-framed messages exert their influence. From an applied perspective, however, it is also important to know under which circumstances gain- or lossframed information is more effective. Future research should thus clarify the circumstances under which one pathway dominates over the other. Perhaps differences in the types of behaviour advocated by the message could influence which affective pathway dominates. For young people, some health-promoting behaviours, like physical activity, might be associated with positive feelings. It might be, then, that for those types of behaviour the positive affective pathway dominates. Other health-promoting behaviours, like breast self-examination, might be associated with negative feelings, like fear. For these types of behaviours, the negative affective pathway might dominate (cf. Rothman \& Salovey, 


\section{Chapter 3}

1997). One additional possibility is that efficacy expectations might be crucial in determining the outcome of message-framing effects. In line with findings from the fear-appeal literature (Witte, 1992; 1994), a mediating effect of negative affect might be most likely when recipients perceive a high efficacy. This might be able to explain the results of a recent study, which found that loss-framed antismoking messages resulted in a stronger intention to quit smoking than gainframed messages, but only for recipients who had high self-efficacy to quit smoking (Van 't Riet et al., 2008). Another possibility is that the likelihood that one pathway dominates over the other depends on recipients' approach and avoidance orientation. Recently, several studies have found that gain-framed information was more persuasive for recipients with a predominant approach orientation (BAS), while loss-framed information was more persuasive for recipients with a predominant avoidance orientation (BIS)(Gerend \& Shepherd, 2007; Mann, Sherman, \& Updegraff, 2004; Updegraff, Sherman, Luyster, \& Mann, 2007). Our results suggest that it would be interesting to investigate whether these findings can be explained by positive and negative affect. There is evidence from a study of persuasion that BAS activity correlates with positive affective responses to a message, while BIS activity correlates with negative emotional responses to a message (Dillard \& Peck, 2001). One could, therefore expect that gain-framed information is more persuasive for recipients with high BAS levels, because, among these recipients, gain-framed information is particularly likely to evoke positive affect. Also, it could be expected that loss-framed information is more persuasive for recipients with high BIS levels, because, among these recipients, loss-framed information is particularly likely to evoke negative affect.

\section{Limitations}

The present research was subject to several limitations. First, because we did not include a no-framing control group, it is difficult to infer whether the gain-framed information increased positive affect or whether the loss-framed information decreased positive affect. However, information that neither stresses the benefits of healthy behaviour, nor the costs of unhealthy behaviour is likely to be incomparable to the health-promoting information we used in the gainframed and loss-framed conditions. This might be one of the reasons why most message-framing studies compare gain- vs. loss-frames and do not include a noframe control group.

Another issue that warrants attention is the fact that we assessed diffuse affect instead of specific emotions. In Study 3.2, four items assessed general positive affective reactions (i.e., happy, relieved, optimistic, content) and four items assessed general negative affective reactions (i.e., sad, afraid, uncomfortable, tense). Doing this, we used what Van der Pligt and colleagues (1998; page 57) called 'a rather crude dichotomy of positive vs. negative affect.' In fact, these 
authors argue that, instead of using these holistic measures, it is essential to distinguish between various, more specific, affective reactions. A limitation of this research, then, is that we did not differentiate more specifically between possibly relevant emotions, like anger, fear, happiness, and relief. Dillard and Peck (2000), for instance, investigated whether the content of eight public service announcements (PSA's) elicited anger, fear, sadness, guilt, contentment, surprise, and happiness. In addition, they investigated which emotions were predictive of perceived effectiveness. The advantage of this approach is that it makes it possible to investigate more specifically which types of information lead to which emotion. It could then be investigated, for instance, whether gain-framed health promoting messages result in happiness, elation, relief, or contentment and whether loss-framed messages result in sadness, disappointment, guilt, or fear. Also, since different emotions are associated with different 'action tendencies' (Frijda, 2007), it might lead to more specific hypotheses concerning which emotions affect behaviour and why. One could hypothesize, for instance, that fear can increase intentions to engage in healthy behaviour, whereas disappointment could lead to decreased motivation. Future research should investigate this possibility. ${ }^{8}$

\section{Conclusion}

Despite the limitations noted above, the results of the present research suggest that both positive and negative feelings may be of great importance in the persuasion process and may be particular helpful to explain the occurrence of framing effects. Whereas positive affect as a result of gain-framed information can contribute to high levels of information acceptance and a positive attitude, thereby exerting a persuasive influence, negative affect as a result of loss-framed information can contribute directly to intention, thereby exerting a motivating influence. These findings suggest that framing exerts influence on persuasion through two different pathways: positive and negative affect. 



\title{
Chapter 4: What Difference Does a Frame Make? Investigating the Influence of Affective Reactions to Skin Cancer Detection Messages ${ }^{9}$
}

\begin{abstract}
Health-promoting messages can be framed in terms of the gains that are associated with healthy behaviour (gain-framed), or the losses that are associated with unhealthy behaviour (loss-framed). In the present research, we examined the role of positive and negative affective reactions in the effects of gainand loss-framed health promoting information. In addition, we investigated whether individual differences in approach and avoidance orientation moderated the effects of message framing. The results of an experiment among 168 university students using gain- and loss-framed messages advocating skin selfexamination showed that gain-framed information resulted in higher levels of positive affect than loss-framed information, whereas loss-framed information resulted in higher levels of negative affect. In addition, positive affect increased recipients' acceptance of the information whereas negative affect increased participants' intention to perform skin self-examination. These results suggest that affect may be helpful to explain the underlying mechanisms of message framing effects. The findings also suggest that gain- and loss-framed messages offer distinct pathways to persuasion. In particular, gain-framed information may exert its effect on recipients through the persuading influence of positive affect, whereas loss-framed information may exert its effect on recipients through the motivating influence of negative affect. No moderating effects of approach orientation or avoidance orientation were found.
\end{abstract}

\section{Introduction}

Skin cancer incidence is increasing rapidly and constitutes a significant health concern. For 2008, the incidence of melanoma, the most dangerous form of skin cancer, was estimated at 62,480 in the United States, with mortality at 8,420 (American Cancer Society, 2008a). Each year, approximately 25,000 individuals are diagnosed with skin cancer in the Netherlands (Dutch Cancer Society, 2006). In light of these figures, early detection of skin-cancer symptoms is of great importance. If cancer is diagnosed early, the patient has a greater chance of successful treatment (American Cancer Society, 2008b). Skin self-examination refers to the act of inspecting one's skin with the aim of detecting possible skincancer related symptoms. Especially when performed frequently, preferably once a month, it can be an effective way to detect skin cancer at an early stage 
(American Cancer Society, 2008b). The present study investigated whether the persuasiveness of communications promoting skin self-examination can be improved by message framing.

Persuasive health messages can be framed in terms of the benefits of engaging in healthy behaviour (gain frame), or in terms of the costs of failing to engage in healthy behaviour (loss frame). Empirical studies have shown that gain- and loss-framed messages can have different effects on individuals' selfprotective actions or motivation to perform healthy behaviours, even when the persuasive information in gain- and loss-framed health messages is factually equivalent (Rothman, Bartels, Wlaschin, \& Salovey, 2006; Rothman \& Salovey, 1997). One influential approach to the study of message framing has been to focus on the function of the recommended behaviour, making a distinction between behaviours that serve to prevent an illness (like exercising or quitting smoking) and behaviours that serve to detect an illness (like skin selfexamination or obtaining a mammography). According to Rothman and Salovey (1997), gain-framed information is more persuasive than loss-framed information when advocating behaviours that serve to prevent illness (i.e., diseaseprevention behaviours). In contrast, they suggest that loss-framed information is more persuasive than gain-framed information for behaviours that serve to detect illness (i.e., disease-detection behaviours). A recent meta-analysis showed, however, that for behaviours serving to detect an illness, gain- and loss-framed messages were not differentially persuasive (O'Keefe \& Jensen, 2006). Thus, in contrast to Rothman and Salovey's reasoning, and despite an impressive body of literature, it is currently unclear how professionals should frame messages promoting active cancer detection in general and skin self-examination in particular.

In the present research, we tried to shed some light on the inconsistent findings in the message-framing literature in two ways. First, we propose that our understanding of framing-effects can be deepened by investigating underlying mechanisms. Because finding no differential effects of message framing can obscure the fact that different, but equally potent mechanisms might underlie the effects of gain- and loss-framed information (Shen \& Dillard, 2007), we tried to investigate the pathways through which gain- and loss-framed information exert their influence on persuasion. Research has shown that gain-framed information evokes positive feelings in the recipient and that loss-framed information evokes negative feelings (e.g., Shen \& Dillard, 2007). In the present paper, we propose that positive and negative affect can both contribute to persuasion, possibly rendering gain- and loss-framed messages equally persuasive. Second, because the message-framing literature does not seem to support the notion that either a gain frame or a loss frame is more persuasive under all or most circumstances, we propose that research investigating possible moderating variables that can explain differences in the effects of gain- and loss-framed messages might also deepen our understanding of message-framing's effects on persuasion. There- 
fore, we tried to investigate under which circumstances gain- or loss-framed information is more persuasive. In the present study, we tested the moderating influence of recipients' individual differences in approach and avoidance orientation (Gray, 1990). In the remainder of the introduction, we will first discuss the role of positive and negative affective reactions. Second, we will outline our hypotheses with regards to approach and avoidance orientations.

\section{The role of affect}

Contemporary theories of emotion state that affect results from an appraisal of the relationship between one's goals and the environment. This appraisal yields positive affect when an individual perceives that the environment is congruent with his or her goals. In contrast, when an individual judges that the environment is in an incongruent relationship with his or her personal goals, the individual experiences negative affect (for overviews see Frijda, 1986; Lazarus, 1991). The purpose of affect is to induce states of 'action readiness' that make the individual responsive to the environment or context (Frijda, 2007). As Shen and Dillard (2007) point out, gain-framed health promoting messages focus on beneficial outcomes, emphasizing possible congruence between the recipient's goals and the environment/situation. Loss-framed health promoting messages focus on costs and by doing so emphasize possible incongruence between goals and the environment/situation. For this reason, we expect gain-framed information to elicit more positive affect than loss-framed information, and loss-framed information to elicit more negative affect than gain-framed information. The limited research that exists on the relationship between framing and affect is consistent with our logic (Chang, 2005; Cox, Cox, \& Zimet, 2006; Cox \& Cox, 2001; Millar \& Millar, 2000; Schneider et al., 2001a; Shen \& Dillard, 2007).

In the present study, we investigated the role of affective reactions to framed health-promoting information. It should be noted that we did not focus on the effects of mood states, but on what Dillard and Meijnders (2002) call 'message induced affect'. Message-induced affect differs from moods (see for instance Bless \& Schwartz, 1999; Forgas, 1995; Isen, Daubman, \& Nowicki, 1987) in that individuals are not always aware of the source of their moods, whereas message-induced affect is most likely to be consciously perceived by recipients as arising from processing the persuasive message (Dillard \& Meijnders, 2002). One theoretical account of the relationship between message-induced affect and persuasion stems from marketing and consumer psychology and is called the 'affect transfer hypothesis' (MacKenzie, Lutz, \& Belch, 1986). According to this account, advertisements that evoke positive affect result in a positive attitude towards the ad, which in turn results in a positive attitude towards the advertised brand. A meta-analysis of persuasion studies in the field of marketing showed support for this hypothesis (Brown, Homer, \& Inman, 1998). 
Negative affect has also drawn attention from persuasion scholars. Numerous studies tested the effects of fear appeals on measures of attitudes, intention and behaviours (for overviews see Eagly \& Chaiken, 1993; Ruiter, Abraham, \& Kok, 2001). A fear appeal is a persuasive message attempting to arouse fear to promote precautionary motivation and self-protective action (Rogers, 1983). Meta-analyses of the fear-persuasion relationship have shown that, under certain circumstances, such as a high perceived efficacy (Witte, 1994), evoking fear can enhance a message's persuasive power (Mongeau, 1998; Ruiter et al., 2001; Witte \& Allen, 2000). With regards to framed messages, the persuasive effects of both positive and negative affect imply that gain-framed messages and loss-framed messages exert their influence on persuasion to a large extent through quite different substrates (positive versus negative affect; see also Cacioppo \& Berntson, 1994).

\section{Previous research}

In two previous experiments (Van 't Riet, Ruiter, Werrij, Candel, \& De Vries, submitted) the hypothesis that positive and negative affect as a result of framed information can both contribute positively to persuasion was investigated. In the first experiment, participants were presented with framed information regarding physical activity after which positive and negative affect and recipients' acceptance of the information was assessed. The results of this experiment revealed that gain-framed information resulted in greater levels of positive affect than loss-framed information, and that positive affect contributed positively to information acceptance. In the second experiment participants were provided with framed messages promoting a low-salt diet. Gain-framed information resulted in greater levels of positive affect than loss-framed information and positive affect contributed positively to information acceptance and attitude. In addition, the results of Study 3.2 revealed that loss-framed information resulted in greater levels of negative affect than gain-framed information, and that negative affect had a significant effect on intention to reduce salt-intake. In sum, whereas positive affect seemed to exert a persuading influence on the recipient, affecting information acceptance and attitude, negative affect seemed to exert a motivating influence on the recipient, having a direct effect on intention. However, physical activity and reducing salt-intake are both prevention behaviours. As mentioned above, Rothman and Salovey (1997) have proposed that the effect of frame is largely dependent on whether the advocated behaviour serves to prevent or to detect illness. For this reason, in the present study, we investigated whether the effects of positive and negative affective reactions could be replicated using messages promoting skin self-examination, a detection behaviour. In addition, we investigated the moderating influence of recipients' chronic approach and avoidance orientations (Gray, 1990). 
Approach/avoidance orientation as a possible moderator of framing effects

According to Gray (1990), two basic mechanisms control human behaviour. One system, the behavioural activation system (BAS), controls appetitive motivation. This system is sensitive to stimuli associated with reward or nonpunishment. The other system, the behavioural inhibition system (BIS), controls aversive motivation and is activated by stimuli of punishment or omission/termination of reward. Gray's bidimensional approach to the regulation of behaviour argues that emotion has an underlying structure that consists of two factors: positive affect and negative affect.

There is evidence from a study of persuasion that BAS activity correlates with positive affective responses to a message, while BIS activity correlates with negative affective responses to a message (Dillard \& Peck, 2001). Other empirical studies (Gerend \& Shepherd, 2007; Mann, Sherman, \& Updegraff, 2004; Sherman, Mann, \& Updegraff, 2006) showed that BAS and BIS activity can moderate the influence of gain- and loss-framed messages on recipients' behaviour. These studies used a questionnaire developed by Carver and White (1994) to measure the strength of participants' approach and avoidance orientation. They then provided participants with framed information. The results of these studies showed that for recipients with a predominant approach orientation, gain-framed information was more persuasive than loss-framed information whereas the reverse was true for recipients with a predominant avoidance orientation. However, participants' affective reactions to the materials were not assessed in these studies, making it impossible to infer whether approach/avoidance orientation also moderated the effects of framing on positive and negative affect. In the present study, we investigated whether BAS and BIS activity moderated the effects of framing on positive and negative affect, and persuasion.

\section{The present study}

We conducted an experiment to test whether framing exerts its effects on persuasion through positive and negative affect. In addition, we investigated whether approach and avoidance orientation could moderate these effects. We used gain- and loss-framed information about skin self-examination as the health-promoting materials. We assessed acceptance of the information, attitude towards skin self-examination and intention to perform skin self-examination as the outcome measures and investigated whether positive and negative affect might influence these outcome measures differently. 


\section{Method}

\section{Participants}

One hundred and sixty-eight students at Maastricht University voluntarily participated in the experiment in exchange for $€ 5$. Most of the participants were female (148 versus 20 males). Age ranged from 17 to 25 years, with a mean age of 20.3 years $(\mathrm{SD}=1.7)$.

\section{Procedure and Design}

This study used a one-factorial (frame: gain frame vs. loss frame) between-participants design. Participants were seated in individual booths and were told that they were about to participate in a study aimed at testing healtheducation materials that had been designed to be used 'on the Internet'. First, the strength of participants' dispositional approach and avoidance orientations were assessed. Next, participants were provided with a persuasive communication about performing skin self-examination. The persuasive communication was either gain- or loss-framed. After reading the persuasive communication, participants completed the dependent measures. Next, participants were debriefed and received their fee.

\section{Assessment of approach and avoidance orientation}

For this study, the Dutch version (Franken, Muris, \& Rassin, 2005) of the BIS/BAS scale (Carver \& White, 1994) was used to assess the strength of people's approach (BAS) and avoidance (BIS) motivations. Participants rated their agreement with 24 statements (including 4 filler items) using Likert scales with endpoints of 1 (strongly disagree) and 4 (strongly agree). The BAS scale consists of three subscales that measure Reward Responsiveness (e.g., 'When good things happen to me, it affects me strongly'), Drive (e.g., 'When I go after something I want, I move on it right away'), and Fun Seeking (e.g., I will often do things for no other reason than they might be fun'). In the present experiment all thirteen BAS items were combined to form an average BAS score (Cronbach's $\alpha=.79$ ) with higher scores indicating stronger BAS sensitivity. The BIS subscale consists of seven items that assess the sensitivity to negative outcomes (e.g., 'I feel worried when I think I have done poorly at something'). All BIS items were combined to create an average BIS score $(\alpha=.84)$ with higher scores indicating stronger BIS sensitivity.

\section{Framed communication}

For the present study, we designed short messages of approximately 200 words, stressing either the benefits of performing skin self-examination (gain frame), or the costs of not performing skin self-examination (loss frame). The full texts are available in Appendix C. 


\section{Outcome measures}

Positive and negative affect. Four items assessed positive affective reactions to the information (positive affect) and four items assessed negative affective reactions (negative affect). The positive affect items assessed the extent to which participants thought the information made them feel happy $(1=$ Very happy; $7=$ Not at all happy $)$, relieved $(1=$ Very relieved $;=$ Not at all relieved $)$, optimistic $(1=$ Very optimistic $; 7=$ Not at all optimistic $)$, and content $(1=$ Very content; $7=$ Not at all content). Scores on the positive affect items were reversed such that high scores indicated high levels of positive affect, and were combined to create an average positive affect score $(\alpha=.84)$. The negative affect items assessed the extent to which participants thought the information made them feel sad $(1=$ Very sad; $7=$ Not at all sad $)$, afraid $(1=$ Very afraid $7=$ Not at all afraid $)$, tense $(1=$ Very tense; $7=$ Not at all tense $)$, and uncomfortable $(1=$ Very uncomfortable $; 7=$ Not at all uncomfortable). Scores on the negative affect items were reversed and combined to create an average negative affect score $(\alpha=.90)$.

Information acceptance. Eight items assessed information acceptance by asking participants to indicate the extent to which they thought the information was convincing $(1=$ Very convincing; $7=$ Not at all convincing $)$, relevant $(1$ $=$ Very relevant $;=$ Not at all relevant $)$, objective $(1=$ Very objective $; 7=$ Not at all objective $)$, useful ( $1=$ Very useful; $7=$ Not at all useful $)$, and interesting ( $1=$ Very interesting; $7=$ Not at all interesting). Furthermore, three items asked participants to indicate the extent to which participants felt that the information was true $(1=$ Very true $; 7=$ Not at all true $)$ and exaggerated $(1=$ Very exaggerated $; 7$ $=$ Not at all exaggerated $)$ and the extent to which participants agreed with the information $(1=I$ totally agree; $7=I$ totally disagree $)$. After we reversed the scores of all items except the exaggerated item, the scores on the eight items were averaged to create an average information acceptance score $(\alpha=.87)$.

Attitude. Five items were used to assess attitude towards performing skin self-examination, asking participants to indicate the extent to which they rated performing skin self-examination as (1) very good vs. (7) very bad, (1) very healthy vs. (7) very unhealthy, (1) very much worth the effort vs. (7) not worth the effort at all, (1) very important vs. (7) very unimportant, (1) very sensible vs. (7) not sensible at all. After scores on the attitude items were reversed, an average score was created $(\alpha=.78)$.

Intention. Intention to engage in skin self-examination was assessed by five items asking participants to indicate whether they agreed with the statements 'I intend to examine my skin once a month in the coming six months', 'I intend to examine my skin once a month in the future', and 'I am thinking about examining my skin once a month', all on 7-point scales $(1=$ I definitely do not agree; $7=I$ definitely agree). In addition, they were asked to indicate how likely it would be that they would examine their skin once a month in the coming six 


\section{Chapter 4}

months, and how likely it would be that they would examine their skin once a month in the future, both on a 7 -point scale $(1=$ Very unlikely; $7=$ Very likely $)$. From these five items an average score was calculated $(\alpha=.94)$.

\section{Statistical analysis}

First, correlations between all relevant variables were investigated. Second, linear regression analyses tested the main effects of frame (coded as $0=$ gain frame, 1 = loss frame), BIS, BAS, and interactions between frame and BIS, and between frame and BAS on positive affect, negative affect, information acceptance, attitude, and intention. To ensure that multicollinearity did not affect the results, individual scores on the BIS and BAS scores were centered (i.e., by subtracting the mean from each score). In case of a significant interaction effect, simple slope analyses were conducted to investigate the nature of the interaction (for a more elaborate description of analyzing interactions in multiple regression, see Aiken \& West, 1991). Third, we performed mediation analyses to investigate whether positive affect mediated between frame and information acceptance and whether negative affect mediated between frame and intention. The used statistical package was SPSS 15.0. Third, relations between relevant variables were investigated using Structural Equation Modelling. For this the statistical package M-Plus 3.11 was used.

\section{Results}

\section{Correlations}

Correlation analyses showed that positive affect was significantly correlated with BIS, information acceptance, attitude, and intention. Negative affect was significantly correlated with BIS and intention (Table 4.1).

\section{Regression analyses}

Linear regression analyses were performed to investigate the effects of frame, BIS, BAS, and the frame by BIS and fame by BAS interaction terms on all outcome measures. In Step 1 of the analyses, we investigated the main effects of frame, BIS, and BAS. In Step 2 of the analyses, we additionally investigated the effects of the frame by BIS and frame by BAS interaction terms. As can be seen in Table 4.2, gain-framed information resulted in higher levels of positive affect than loss-framed information, whereas loss-framed information resulted in more negative affect than gain-framed information. In addition, participants with a strong BIS reported higher levels of negative affect than participants with a weak BIS. No other significant effects were found (see Table 4.2 and Table 4.3). 
Table 4.1 Correlations between BIS, BAS, positive affect, negative affect, information acceptance, attitude and intention

\begin{tabular}{lllllll}
\hline & BIS & BAS & $\begin{array}{l}\text { Positive } \\
\text { Affect }\end{array}$ & $\begin{array}{l}\text { Negative } \\
\text { Affect }\end{array}$ & $\begin{array}{l}\text { Information } \\
\text { Acceptance }\end{array}$ & Attitude \\
\hline BIS & - & & & & & \\
BAS & .07 & - & & & & \\
Positive Affect & $-.17^{*}$ & .07 & - & & & \\
Negative Affect & $.29^{* *}$ & .06 & $-.37^{* *}$ & - & & \\
Information Acceptance & .12 & .03 & $.17^{*}$ & .12 & - & $.61^{* *}$ \\
Attitude & .07 & .11 & $.19^{*}$ & .08 & $.52^{* *}$ & $.77^{* *}$ \\
Intention & .04 & .11 & $.18^{*}$ & $.16^{*}$ & & \\
\hline
\end{tabular}

$* \mathrm{p}<.05$

$* * \mathrm{p}<.01$

\section{Mediation analyses}

Mediation analyses were performed to investigate whether positive affect mediated between frame and information acceptance and whether negative affect mediated between frame and intention. For this purpose, we conducted a test of the joint significance of the two effects comprising the mediation effect (MacKinnon, Lockwood, Hoffman, West, \& Sheets, 2002). In this approach, a variable functions as a mediator when two conditions are met: (a) the independent variable has a significant effect on the mediator (Path A), and (b) the mediator is significantly associated with the dependent variable when the other independent variables are controlled for (Path B). In the commonly used Baron and Kenny approach (Baron \& Kenny, 1986), two other conditions are posited: (1) the independent variable has a total effect on the dependent variable and (2) this total effect is reduced when the mediator is entered into the model. However, the test of joint significance has been shown to have greater power than the traditional Baron and Kenny approach (MacKinnon et al., 2002).

With regards to the mediating role of positive affect, Table 4.2 shows that frame had a significant effect on positive affect (Path A). To test whether positive affect had a significant effect on information acceptance while frame was controlled for (Path B), we conducted another linear regression with frame and positive affect as the independent variables and information acceptance as the dependent variable. In this analysis, positive affect had a significant effect on information acceptance, $B=.18, t(165)=1.99, p<.05$. This supported our hypothesis and suggests that positive affect mediated between frame and information acceptance. A Sobel's Z test (Sobel, 1982) revealed that the mediation was marginally significant, $Z=1.68, p=.09$.

With regards to the mediating role of negative affect, Table 4.2 shows that frame had a significant effect on negative affect (Path A). To test whether 


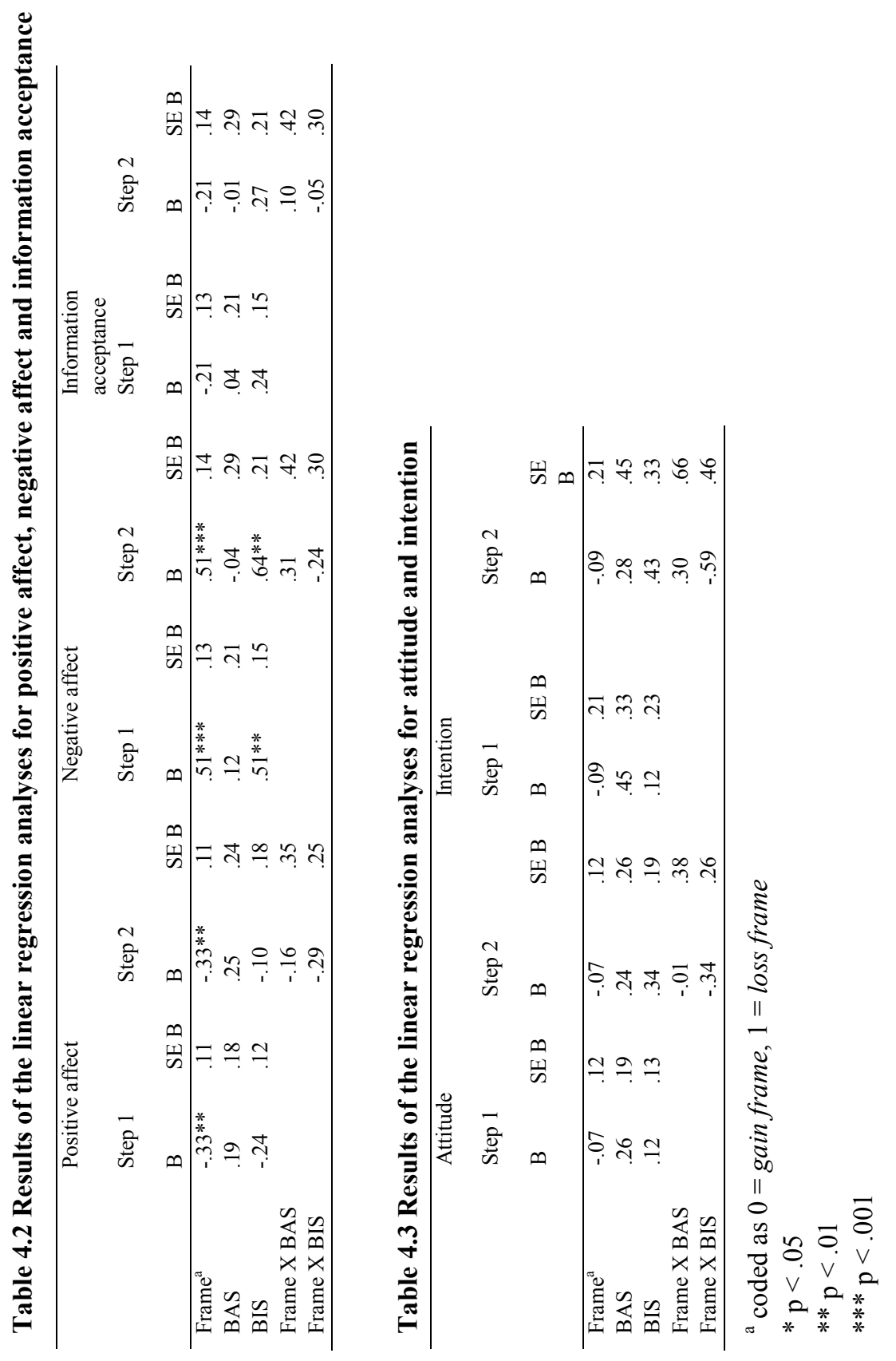


negative affect had a significant effect on intention while frame was controlled for (Path B), we conducted another linear regression with frame and negative affect as the independent variables and intention as the dependent variable. In this analysis, negative affect had a significant effect on intention, $B=.27, t(165)$ $=2.33, p<.05$. This supported our hypothesis and suggests that negative affect mediated between frame and intention. A Sobel's $\mathrm{Z}$ test revealed that the mediation was statistically significant, $\mathrm{Z}=2.02, p<.05$.

\section{Path Model}

Previous research has shown that positive and negative affect can influence information acceptance, attitude and intention differently (Van 't Riet et al., submitted). For this reason, we propose that these three outcome measures should not be perceived as separate parts of a single 'persuasion' construct. Rather, we constructed a path model in which higher levels of information acceptance lead to a more positive attitude and a more positive attitude in turn leads to a stronger intention. Next, we tested a path model in which frame furthermore predicts positive and negative affect, positive affect predicts information acceptance and attitude, and negative affect has a direct effect on intention. Based on the correlation analysis (see Table 4.1), a correlation between positive affect and negative affect was added to the model. The analysis showed that the path from positive affect to attitude was non-significant. Thus, this path was omitted from the model. Figure 4 shows the path model in which frame predicts positive and negative affect, positive affect predicts information acceptance, and negative affect has a direct effect on intention. Analyses using Structural Equation Modeling show that this model fitted the data well, $\chi^{2}(16)=24.741, p=$ $.0745 ; \mathrm{CFI}=.971 ; \mathrm{RMSEA}=.057, \mathrm{SRMR}=.056$. To obtain an additional test of our model, an extended model was tested in which frame predicted positive and negative affect, BAS predicted positive affect, BIS predicted negative affect, and frame, BAS, BIS, positive affect, and negative affect all predicted information acceptance, attitude, and intention. This model also included paths from information acceptance to attitude and intention, and from attitude to intention. This extended model did not have a significantly greater model fit than the model in Figure $4, \Delta \chi^{2}(13)=19.932, p=$ n.s. The paths from frame, BAS, and BIS to information acceptance, attitude and intention were not significant, and neither were the paths from positive affect to attitude and intention, or the path from negative affect to information acceptance and attitude. 
Figure 4 Path model showing positive affect as a predictor of information acceptance and negative affect as a predictor of intention"

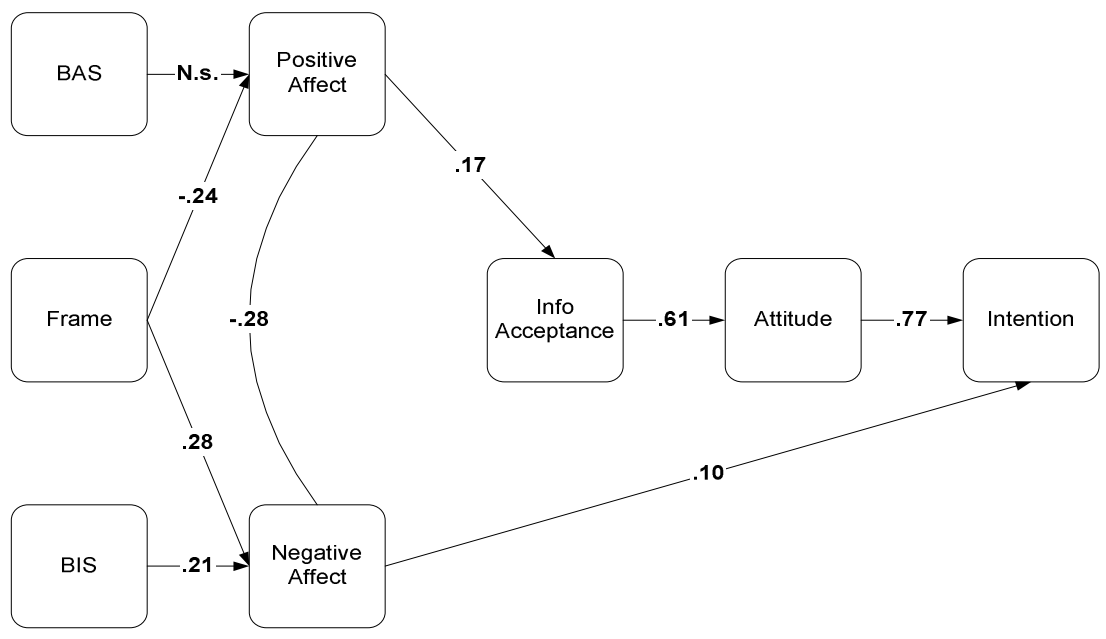

* All paths are significant at the $\mathrm{p}=.05$ level, except if indicated otherwise

\section{Discussion}

The aim of the present study was to investigate whether information that is gain- versus loss-framed gives rise to different levels of positive and negative feelings and whether these feelings exert an influence on persuasion. Additionally, we investigated whether dispositional approach and avoidance orientations could moderate the effect of framing on positive and negative feelings and other outcome measures. The results showed that gain-framed information evoked greater levels of positive affect in recipients than loss-framed information and that positive affect in turn was positively associated with information acceptance. The results furthermore showed that loss-framed information resulted in greater levels of negative affect than gain-framed information and that negative affect, while not being associated with information acceptance or attitude, had a significant effect on intention to perform skin self-examination. No moderating effects of approach or avoidance orientation were found.

The results of the present study replicate those of two previous studies (Van 't Riet et al., submitted) that suggest that positive affect exerts a persuading influence on the recipient, affecting information acceptance, whereas negative affect exerts a motivating influence on the recipient, having a direct effect on intention. Whereas these previous studies used framed information about disease-prevention behaviours (i.e., physical activity and reducing salt-intake), the results of the present study suggest that similar mechanisms are at work for mes- 
sages concerning disease-detection behaviours. Also, the results are in accordance with research by Fredrickson and Losada (2005) that suggests that both positive and negative emotions have adaptive value, but in a different way. Positive emotions can broaden people's mindsets, expanding cognition and behavioural tendencies (Fredrickson \& Branigan, 2005). This may account for the fact that, in the present study, positive affective reactions influenced information acceptance; positive affect may have rendered participants more 'open' to the health-promoting information. Negative emotions, on the other hand, narrow people's behavioural urges toward specific actions, preparing the body physically and mentally for immediate action (Fredrickson and Losada, 2005; see also Frijda's (2007) conceptualization of action readiness). Thus it is not surprising that negative affect as a consequence of loss-framed information exerted a motivating influence more directly on intention.

Even though gain-framed information elicited higher levels of positive affect than loss-framed information and positive affect was associated with information acceptance, in contrast to our previous studies this did not result in greater information acceptance ratings for the gain-framed messages. In fact, we only found significant effects of frame on positive and negative affect and did not find any other framing effects, either alone or in combination with approach and avoidance orientations. In this regard, the present study might be exemplary for the message-framing literature as a whole. Besides the occasional nullfindings that have been reported (Brug, Ruiter, \& Van Assema, 2003; Jones, Sinclair, Rhodes, \& Courneya, 2004), recent meta-analyses provide strong evidence that gain- and loss-framed messages do not affect psychological and behavioural outcomes in a consistent and predictable way across all populations (O'Keefe \& Jensen, 2006; 2007). The results of the present study contribute to the literature, however, by showing that underneath these rather discouraging null-findings two distinct processes may be at work. As Shen and Dillard (2007) point out, focusing only on the outcomes of message-framing effects and ignoring underlying mechanisms obscures the possibility that gain- and loss-framed messages exert their influence on persuasion through different pathways. This way researchers might be unable to reveal effects that can be relevant to healthpromotion professionals.

In contrast to a number of recent studies (Gerend \& Shepherd, 2007; Mann, Sherman, \& Updegraff, 2004; Sherman, Mann, \& Updegraff, 2006) we did not find evidence for an interaction between framing and approach/avoidance orientations. It could be that our participants' motivation to process the framed messages might have influenced the results (Chaiken, Liberman, \& Eagly, 1989; Petty \& Cacioppo, 1986). Research has shown that congruence between recipients' motivational orientation and the message frame (i.e. when predominantly approach oriented recipients receive gain-framed information or when predominantly avoidance oriented recipients receive loss-framed information) contributes 
to persuasion by increasing recipients' motivation to elaborate on the message content (Updegraff, Sherman, Luyster, \& Mann, 2007). Our sample, however, was predominantly female and previous research suggests that women are more involved in the issue of skin cancer than are men (Rothman, Salovey, Antone, Keough, \& Drake Martin, 1993). Thus, in the present study, many participants might have already been highly motivated to process the message thoroughly and systematically, regardless of motivational orientation - message frame congruence. This way congruence might not have had an additional effect.

The present study was subject to certain limitations. First, as mentioned above, our sample was predominantly female. Future studies should investigate whether the same results can be obtained by a more balanced or a male sample. Second, our study did not include a long-term follow-up, making it impossible to assess behavioural effects. Future research should investigate the long-term behavioural effects of framing or should use proximal measures of behaviour, like willingness to register for a course in skin self-examination (cf. Ruiter, Verplanken, De Cremer, \& Kok, 2004) or willingness to fill in a coupon to receive additional information (cf. Detweiller, Bedell, Salovey, Pronin, \& Rothman, 1999).

Despite these limitations, the results of the present research suggest that both positive and negative feelings may be of great importance in the persuasion process and may be particularly helpful to explain the occurrence of framing effects. Whereas positive affect as a result of gain-framed information can contribute to high levels of information acceptance, negative affect as a result of loss-framed information can contribute directly to intention. These findings suggest that framing exerts influence on persuasion through two different pathways: positive and negative affect. 
Part 3

Moderating Variables 



\title{
Chapter 5: The Influence of Self-Efficacy on the Effects of Framed Health Messages ${ }^{10}$
}

\begin{abstract}
Health-promoting messages can be framed in terms of the gains that are associated with healthy behaviour or the losses that are associated with unhealthy behaviour. In this study we examined the influence of self-efficacy to quit smoking on the effects of gain- and loss-framed anti-smoking messages in a randomized controlled trial among 539 adult smokers. Participants with high self-efficacy to quit smoking reported higher levels of motivation to quit smoking after receiving a loss-framed message than after receiving a gain-framed message or no message. For these participants receiving a gain-framed message did not result in a higher motivation to quit smoking than receiving no message. For participants with a low self-efficacy to quit smoking there were no differences in motivation to quit smoking between the gain-framed message condition, loss-framed message condition and control condition. Our results suggest that self-efficacy can moderate the effects of message framing on persuasion.
\end{abstract}

\section{Introduction}

Persuasive health messages are a central component of efforts to promote healthy behaviour. Hence research investigating the mechanisms underlying their (in)effectiveness is important. The present study focuses on how persuasive health messages are framed. Persuasive health messages can be framed in terms of the benefits of engaging in healthy behaviour (gain-framed) or in terms of the costs of failing to engage in healthy behaviour (loss-framed). Empirical studies have shown that gain- and loss-framed messages have different effects on individuals' self-protective action or motivation to perform healthy behaviour, even when the information contained in gain- and loss-framed persuasive health messages is factually equivalent (Rothman \& Salovey, 1997). Research investigating possible moderating variables that can explain differences in effects of gain- and loss-framed messages is therefore indispensable to foster our understanding of the effects of message framing on persuasion.

One factor that may influence the effects of framed health-promoting messages is the perceived risk of the goal behaviour. Research conducted by Kahneman and Tversky (Kahneman, 2003; Kahneman \& Tversky, 1984) showed that people can respond differently to information about the consequences of a risky choice depending on whether the same consequences were presented as gains or losses. Specifically, Kahneman and Tversky proposed that loss-framed information coins people to prefer taking risks to options that are certain. Gain- 
framed information, on the other hand, coins people to prefer certainty to risk. These findings have been extended to persuasion research (Banks, Salovey, Greener, \& Rothman, 1995; Cherubini, Rumiati, Rossi, Nigro, \& Calabro, 2005; Meyerowitz \& Chaiken, 1987; Rothman, Bartels, Wlaschin, \& Salovey, 2006; Rothman \& Salovey, 1997; Rothman, Salovey, Antone, Keough, \& Drake Martin, 1993). Rothman and Salovey (1997), for instance, proposed that the influence of a framed health promoting message is contingent on the degree to which performing the promoted health behaviour is perceived as risky. They state that people perceive behaviours that serve to prevent illness, like quitting smoking or exercising, as risk-free behaviours because performing them does not result in any increased health risk but will reduce it. In contrast, behaviours that serve to detect illness, like breast self examination, will be perceived as risky because performing them entails the risk of discovering a disease (Rothman et al., 2006; Rothman et al., 1993). In line with the postulates of Prospect Theory (Kahneman, 2003; Kahneman \& Tversky, 1984), Rothman and Salovey (1997) propose that gain-framed messages will be more effective than loss-framed messages in motivating people to engage in (risk-free) prevention health behaviours and lossframed messages will be more effective than gain-framed messages in motivating people to engage in (risky) detection health behaviours.

However, empirical findings have not always been consistent with this view. For example, Blanton and colleagues found that loss-framed messages were more persuasive than gain-framed messages in promoting condom use (Blanton et al., 2001). Also, Cox and colleagues found gain-framed messages to be more persuasive than loss-framed messages for promoting skin selfexamination (Cox, Cox, \& Zimet, 2006).

In response to Rothman and Salovey's reasoning, Levin and colleagues (1998) have proposed three different types of framing: risky choice framing, attribute framing, and goal framing. In risky choice framing, two potential consequences of a choice, differing in risks, are described in terms of gains or losses. This is the framing that is most closely associated with the research by Kahneman and Tversky. For risky choice framing, Levin and colleagues accept that loss-framed information leads people to prefer taking risks to options that are certain, whereas gain-framed information leads people to prefer certainty over risks. The second kind of framing, attribute framing, consists of describing a characteristic of an object or event either in positive or negative terms, for example describing the consequences of a medical procedures as having a $90 \%$ survival rate, or, alternatively, as having a $10 \%$ mortality rate. According to Levin and colleagues, objects or events are generally rated as more attractive when their characteristics are described in a positive way, rendering a gain frame more effective. The third kind of framing, goal framing, refers to manipulating persuasive communications to stress either the advantages of a certain behaviour or the disadvantages of not performing that behaviour. With regards to goal framing, 
Levin and colleagues state that loss-framed messages are generally more persuasive than gain-framed messages. A similar distinction between different kinds of framing has been proposed by Edwards and colleagues (2001), who conclude from their systematic review of framing effects that loss-framed information was generally more effective than gain-framed information when it comes to persuading individuals to behave in a healthy way.

A 'negativity bias' could be a possible explanation for this greater persuasiveness of loss-framed messages. The term 'negativity bias' refers to the phenomenon that people sometimes pay more attention to negative information than to factually equivalent positive information (Fiske, 1980; Kanouse, 1984). Because people perceive the world as predominantly positive, negative information will trigger more attention (Lau, 1985). Increased attention can lead to more systematic processing, which, in case of convincing arguments, leads to more persuasion (Chaiken, Liberman, \& Eagly, 1989). For this reason, messages advocating healthy behaviour may be more effective when framed in terms of losses instead of gains (Levin et al., 1998; Meyerowitz \& Chaiken, 1987). However, many studies have found gain-framed messages to be more effective than loss-framed messages (e.g., Detweiller, Bedell, Salovey, Pronin, \& Rothman, 1999; Schneider et al., 2001b; Wong \& McMurray, 2002), which argues against the negativity bias. Since there is no definitive explanation for framing effects yet, an important conclusion is that research is needed to identify possible moderating variables that can explain differences in effects of gain- and loss-framed messages (see for instance Broemer, 2002; 2004).

Research on fear appeals suggests that persuasive messages containing negative information may be effective, to the extent that the negative information increases recipients' perception of threat (Witte, 1992). According to Witte, at least some level of perceived threat is needed for recipients to be motivated to pay attention to the message. However, Witte (1992) also noticed that whereas some studies seem to underline the effectiveness of fear appeals, other studies demonstrate their ineffectiveness and concluded that 'empirical findings are disappointingly inconsistent, if not contradictory' (p. 329; see also Ruiter, Abraham, \& Kok, 2001). In an attempt to explain both acceptance and rejection of fear appeals, Witte (1992) proposed the Extended Parallel Process Model (EPPM). The EPPM proposes that high levels of threat combined with high perceived efficacy yields 'danger control', an adaptive cognitive process in which people evaluate the threat and the suggested coping response and which most likely leads to acceptance of the recommended action. Thus, a greater perceived threat will likely result in greater message effectiveness when people think that they have the ability to avert the threat (see also Leventhal, 1970, for a review see Ruiter et al., 2001). In contrast, high levels of threat and low perceived efficacy yields 'fear control', which consists of efforts to reduce the unpleasant experience of fear by means of avoidance or denial of the message. Thus, if people 
think they lack the skills to avert the threat, they may be motivated to respond defensively to the negative message (Stephenson \& Witte, 1998; Witte, 1992). Perceived efficacy consists of response efficacy (the perception that the recommended action can avert the threat; Rogers, 1975) and self-efficacy (confidence in the ability to perform the recommended behaviour; Bandura, 1986). Thus, for persuasive messages to be effective, both the perception of a certain level of threat and confidence in one's ability to avert the threat seem important.

These predictions concerning danger control and fear control might be able to explain why in some cases loss-framed messages are more persuasive than gain-framed messages. Loss-framed messages have been found to evoke a greater sense of threat than gain-framed messages (Cox \& Cox, 2001; Shen \& Dillard, 2007), and this greater sense of threat might increase a message's persuasiveness. However, when recipients do not have sufficient confidence in their ability to avert the threat, the greater sense of threat that is conveyed in lossframed messages as compared to gain-framed messages might result in defensive processing. This might explain why in some cases loss-framed messages are not more persuasive than gain-framed messages.

In sum, loss-framed messages may be more persuasive than gainframed messages because loss-framed messages convey a greater sense of threat (Cox \& Cox, 2001; Shen \& Dillard, 2007). This advantage of loss-framed information may be particularly pronounced when recipients have confidence that the threat can be averted. For recipients with little confidence that the threat can be averted, we do not expect loss-framed information to be more persuasive, because the greater sense of threat that is conveyed by loss-framed information might give rise to defensive processing (Stephenson \& Witte, 1998; Witte, 1992).

In the present study we tested these assumptions using framed antismoking messages which were targeted at current smokers. We focused on selfefficacy to quit smoking instead of response-efficacy of smoking cessation because knowledge about the beneficial effects of smoking cessation is widespread. Therefore, we did not expect large differences in response-efficacy among participants.

Self-efficacy (Bandura, 1986) is a major determinant of motivation to engage in healthy behaviour (De Vries \& Backbier, 1994; Schwarzer, 1992; Strecher, DeVellis, Becker, \& Rosenstock, 1986). Self-efficacy beliefs have been found to be associated with smoking intentions in youth (Choi, Gilpin, Farkas, \& Pierce, 2001; Tucker, Ellickson, \& Klein, 2002), with success in quitting smoking in adults (Baer, Holt, \& Lichtenstein, 1986; DiClemente, Prochaska, \& Gilbertini, 1985), and with avoiding relapse (Gwaltney et al., 2001). Given the importance of the self-efficacy construct in the investigation of health behaviour it is worthwhile to investigate the possible influence of self-efficacy on framing 
effects. To our knowledge, this is the first study that reports on the moderating role of self-efficacy in message framing effects.

\section{The Present Study}

We hypothesize that for participants high in self-efficacy a loss-framed message is more persuasive than a gain-framed message because loss-framed information conveys a greater sense of threat than gain-framed information and this greater threat results in a greater motivation to change current behaviour. For participants low in self-efficacy we predict that a loss-framed message is not more persuasive than a gain-framed message because the greater sense of threat conveyed by the loss-framed message as compared to the gain-framed message coins recipients who are low in self-efficacy to react defensively.

\section{Method}

\section{Procedure and Design}

Data were gathered at various markets and fairs. A booth was set up at each event and visitors were asked to participate in the study. Current smokers of at least 18 years old were eligible to participate in the study. Individuals who agreed to participate were randomly assigned to one of three experimental conditions: gain-framed information, loss-framed information and no information. Participants received a booklet that consisted of a pretest questionnaire, followed by the experimental manipulation (either a gain-framed written anti-smoking message, a loss-framed written anti-smoking message or no message), and a post-test questionnaire. Participants were asked to answer the questions on the first pages, read the text carefully, and finally answer the questions on the last pages. Participants could read the text and complete the questionnaires immediately. Those who completed the study could win one out of 30 prices of 30 euros each by means of a lottery. Participants were informed that their data would be treated confidentially, and that they could refrain from participation at any moment during the experiment. The study used a one-factorial (framing: gainframed message condition, loss-framed message condition, control condition) between-participants design.

\section{Materials}

Pre-test questionnaire. The pretest questionnaire assessed demographic characteristics of the sample, number of cigarettes smoked per day and number of quit attempts in the past year. Furthermore, motivation to quit smoking at baseline was assessed with one question that was based on the Stage of Change construct from the Trans Theoretical Model (Prochaska et al., 1994) and has been used in previous studies (Bolman \& De Vries, 1998; De Vries, Mudde, 
Dijkstra, \& Willemsen, 1998; Dijkstra, Roijackers, \& De Vries, 1998). The question had seven answering options: (1) intending to keep smoking without cutting back on cigarettes and without trying smoking lighter cigarettes; (2) intending to keep smoking, but intending to cut back on cigarettes or try smoking lighter cigarettes; (3) intending to quit at some point, but not within five years; (4) intending to quit within five years, but not within one year; (5) intending to quit within one year, but not within six months; (6) intending to quit within six months, but not within one month; or (7) intending to quit within one month.

Ten questions assessed self-efficacy. These items were based on earlier work on smoking cessation (Bolman \& De Vries, 1998; De Vries et al., 1998; Dijkstra et al., 1998). All items used 7-point scales. The first question assessed whether participants judged quitting smoking hard or easy $(1=$ very hard, $7=$ very easy). The second question assessed whether participants thought they would succeed if they would try to quit smoking in the next six months $(1=n o$, certainly not, 7 = yes, certainly). The next eight questions assessed whether participants thought they would be able to refrain from smoking in eight different situations: when sad, when startled, when friends would smoke, when drinking alcohol, when present at a party, when bored, shortly after eating, when taking a break $(1=$ no, certainly not, $7=$ yes, certainly $)$. The responses on the selfefficacy items had good internal consistency $(\alpha=.90)$. The scores on the ten items were therefore averaged to create one self-efficacy score.

Persuasive communication. The persuasive communication consisted of a short message about either the positive consequences of quitting smoking or the negative consequences of continuing smoking. In the gain-framed condition the text was entitled 'Why is it good to quit smoking?'. The text contained statements such as '[If you quit smoking,] your risk of lung cancer will decrease' and '..., you will stop spending a lot of money on cigarettes'. The gain-framed message contained 176 words. In the loss-framed condition the text was entitled 'Why is it bad to continue smoking?', and contained statements such as: '[If you continue smoking,] your risk of lung cancer will increase' and '..., you will spend a lot of money on cigarettes'. The loss-framed message contained 172 words. The full texts are available in Appendix D.

Manipulation check. The post-test questionnaire contained a check of the framing manipulation. One item assessed whether participants perceived the tone of the message as negative versus positive on a 7-point semantic differential $(1=$ very negative , $7=$ very positive $)$. Participants in the control condition did not receive this manipulation check.

Precautionary motivation. At post-test, motivation to quit smoking was measured with three questions on a 7-point scale with endpoints (1) certainly not and (7) certainly that assessed whether participants planned to quit smoking within one month, within six months, and whether they planned to ever quit. Responses on these items were significantly correlated and had acceptable inter- 
nal consistency $(\alpha=.79)$. One composite motivation score was therefore created by averaging the scores on the three items. The assessment of post-test motivation differed from the assessment of pre-test motivation to avoid a simple repetition effect.

\section{Analysis Overview}

Regression analyses tested the main effects of frame (using two dummy variables, 'gain frame' was coded as $0=$ control / loss frame, 1 = gain frame; 'loss frame' was coded as $0=$ control / gain frame, $1=$ loss frame) self-efficacy and the two-way interactions between both framing dummy variables and selfefficacy. We controlled for pre-test motivation to quit smoking and for the pretest number of cigarettes smoked per day by using these variables as covariates in the analyses. To ensure that multicollinearity did not affect the results, individual scores on the self-efficacy measure, pre-test motivation, and pre-test number of cigarettes were centered (i.e., by subtracting the mean from each score). In case of a significant interaction effect, simple slope analyses were conducted to investigate the nature of the interaction (for a more elaborate description of analyzing interactions in multiple regression, see Aiken \& West, 1991).

\section{Results}

\section{Participant Characteristics.}

A total of 592 participants participated in this study. After excluding participants who had missing values on $10 \%$ or more of the variables, 539 participants were included in the analysis. The sample consisted of $58.4 \%$ men $(\mathrm{n}=$ $315)$. Furthermore, $20.2 \%(\mathrm{n}=109)$ of the participants had a low education level, $59.4 \%(n=320)$ had a medium level of education, and $16.5 \%(n=89)$ had a high level of education. In the complex schooling system in the Netherlands, a low education level refers to primary or basic vocational school, a medium education level refers to secondary vocational school or high school, and a high education level refers to advanced vocational school or university. The education level could not be assessed for 21 participants due to missing values. Age ranged from 18 to 77 years, with a mean of 42.7 years $(S D=13.3)$. Participants reported smoking an average of 18.6 cigarettes a day, ranging from 1 to 60 . The demographic and smoking behaviour profile of the participants is summarized in Table 5. The mean self-efficacy score across all participants was 3.1 ( $\mathrm{SD}=1.4$ ). At baseline, self-efficacy was negatively correlated with the amounts of cigarettes smoked per day, $r=-.17, p<.001$, and positively correlated with motivation to quit smoking, $r=.27, p<.001$. There was no significant correlation between selfefficacy and the number of quit attempts in the past year, $r=-.04, p=.33$. Regression analyses with the two framing dummy variables as the independent 
variables and pre-test motivation and pre-test number of smoked cigarettes as the dependent variables revealed that respondents in the gain-framed message condition, loss-framed message condition and control condition did not differ in pretest motivation, $p \mathrm{~s}>.63$, or pre-test number of smoked cigarettes per day, $p \mathrm{~s}>$ .42 .

\section{Manipulation Check.}

To test whether the manipulation of frame was successful scores of participants in the gain- and loss-framed message condition were analyzed using a regression analysis with one framing dummy variable (coded as $0=$ loss frame, 1 = gain frame), self-efficacy and the frame $\mathrm{X}$ self-efficacy interaction term as the independent variables and message tone as the dependent variable. This analysis revealed that participants exposed to the gain-framed information judged the information to be significantly more positive, $B=.67, t(347)=4.00, p<.001$. No other effects were found, $p \mathrm{~s}>.14$.

\section{Precautionary Motivation.}

Regression analysis tested the effects of loss frame, gain frame, selfefficacy, the loss frame $\mathrm{X}$ self-efficacy interaction term and the gain frame $\mathrm{X}$ self-efficacy interaction term on the post-test motivation to quit smoking using pre-test motivation and the pre-test number of cigarettes smoked per day as covariates. The analysis revealed that the loss frame by self-efficacy interaction term had a significant contribution to the prediction of post-test precautionary motivation, $B=.13, t(477)=1.93, p=.05$. The gain frame by self-efficacy interaction effect was not significant, $B=.10, t(477)=1.33, p=.19$. Simple slope analyses were conducted to investigate the influence of message framing for participants with a low self-efficacy (i.e., one SD below the mean) and participants with a high self-efficacy (i.e., one SD above the mean). Simple slope analysis revealed that, for participants with a low self-efficacy, there were no significant differences in motivation to quit smoking between the three conditions, $p \mathrm{~s}>.18$. However, participants with a high self-efficacy had significantly higher ratings of post-test motivation to quit smoking in the loss-framed message condition as compared to the gain-framed message condition, $B=.29, t(477)=2.09, p<.05$, and as compared to the control condition, $B=.45, t(477)=3.41, p=.001$. There was no difference in post-test precautionary motivation between high selfefficacy participants in the gain-framed message condition and those in the control condition, $B=.15, t(477)=1.06, p=.29($ see Figure 5$)$. 
Table 5 Demographic profile of participants

\begin{tabular}{ll}
\hline Variable & Percentage \\
\hline Sex & \\
Male & 58.4 \\
Female & 41.6 \\
Education & \\
Low & 20.2 \\
Average & 59.4 \\
High & 16.5 \\
Cigarettes smoked & \\
1-5 & 4.9 \\
6-10 & 17.3 \\
11-15 & 23.7 \\
16-20 & 27.9 \\
21 or higher & 26.2 \\
Intention to quit & \\
Not quitting, not cutting back & 16.8 \\
Not quitting, cutting back & 11.5 \\
Not quitting in the next 5 years & 20.0 \\
Not quitting in the next year & 21.1 \\
Not quitting in the next six month & 13.8 \\
Not quitting in the next month & 9.6 \\
Quitting in the next month & 7.2 \\
\end{tabular}

To assess whether the frame by self-efficacy interaction had a similar effect on motivation to quit smoking within one month, within three months and on motivation to ever quit, separate regression analyses were performed for each of the three post-test motivation items. These analyses revealed that, for the item that assessed whether participants intended to quit smoking within one month, there was a marginally significant loss frame by self-efficacy interaction effect, $B$ $=.18, t(487)=1.90, p=.06$. For the item that assessed whether participants intended to quit smoking within six months, the loss frame by self-efficacy interaction term also had a marginally significant contribution to the prediction of motivation, $B=.18, t(482)=1.91, p=.06$. For these two items, simple slope analyses yielded largely the same pattern of results as for the composite post-test motivation scale. For the item that assessed whether participants intended to ever quit, there was no significant loss frame X self-efficacy interaction effect, $B=.07$, $t(493)=.64, p=.52$. 
Figure 5 Regression slopes arising from the relation between pre-test selfefficacy and precautionary motivation for participants in the gain-framed message condition, the loss frame message condition, and the control condition

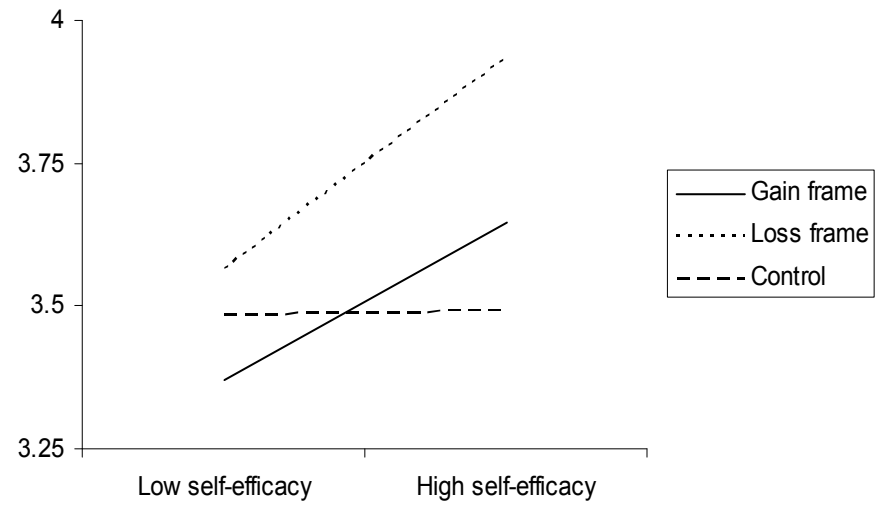

\section{Discussion}

Our results supported our hypothesis concerning differential effects of framing for respondents with high and low levels of self-efficacy. We proposed that loss-framed messages would be more persuasive than gain-framed messages because of the greater threat they entail. We also hypothesized that this advantage of loss-framed messages would be particularly pronounced for recipients with a high self-efficacy to avert the threat. When self-efficacy is low, threatening health promoting messages might be processed defensively. As such, the negative information in loss-framed messages might be avoided or dismissed, and as a result, loss-framed messages might lose their advantage over gainframed messages.

We found that participants with high self-efficacy to quit smoking who read a loss-framed anti-smoking message were more motivated to quit smoking at the post-test than participants with high self-efficacy who read a gain-framed message and participants who read no message. For participants with low selfefficacy to quit smoking, we found no differences in post-test motivation to quit smoking between the gain-framed message condition, loss-framed message condition and control condition.

In organizational psychological research, studies have shown that people with high levels of self-efficacy set higher goals (Locke \& Latham, 2002) and display greater task persistence (Bandura \& Cervone, 1986). In health behaviour research, self-efficacy has been found to be a major determinant of mo- 
tivation to engage in healthy behaviour (De Vries \& Backbier, 1994; Schwarzer, 1992; Strecher et al., 1986). The results of the present study show that selfefficacy can also influence the differential effectiveness of gain and loss-framed health promoting messages on precautionary motivation.

Questions remain as to which factors influenced the self-efficacy of participants in our sample. We reported a negative (but weak) correlation between number of cigarettes smoked per day and self-efficacy to quit smoking. It is possible that smokers in our sample had a higher self-efficacy to quit smoking to the extent that they had already been successful in reducing the number of cigarettes per day.

We suggested that self-efficacy would influence the extent to which the processing of threatening information was defensive because people with high self-efficacy have confidence that they can avert the threat. An additional explanation for the relationship between self-efficacy and defensive processing relation can be found in the literature on self-affirmation (Reed \& Aspinwall, 1998). According to this view, information about changing health behaviour is not only threatening because it contains information about physical threats, such as lung cancer or cardiovascular disease, it also poses a threat to the receiver's selfimage. This threat to the self can lead people to process threatening information defensively (Sherman, Nelson, \& Steele, 2000). However, when people feel good about themselves a threat to the self does not seem to result in defensive message processing. In a study by Reed and Aspinwall (1998), for instance, high-relevance participants who had just recalled a positive experience processed health-risk information less defensively. Moreover, Reed and Aspinwall found that self-affirmed participants reported a greater perceived behavioural control, a construct similar to self-efficacy (Ajzen, 2002b). It could well be that the association between positive experiences and self-efficacy also works the other way around and that self-efficacy gives rise to positive feelings and a general sense of self-esteem (Schwarzer, 1992). Thus, when it comes to processing health promoting messages, receivers' sense of self-efficacy could serve as a selfaffirmation, making message processing less defensive.

One other issue warrants attention. According to Rothman and Salovey (1997), gain-framed messages are more effective when it comes to promoting behaviour that serves to prevent illness. Smoking cessation can be construed as a preventive behaviour, yet our results show that the participants who had the highest scores on post-test motivation to quit smoking were the participants with a high self-efficacy who had read the loss-framed anti-smoking message. Thus, the results of the present study raise questions on Rothman and Salovey's theorizing.

This study had limitations that need to be addressed. First, questions remain as to why participants with high self-efficacy levels reported a higher motivation to quit smoking after reading a loss-framed message than after read- 


\section{Chapter 5}

ing a gain-framed message. We suggested that, for highly self-efficacious participants, loss-framed messages would be more persuasive, because they convey a greater sense of threat, whereas for participants with low levels of self-efficacy, loss-framed messages would be less persuasive because for those participants a greater sense of threat may cause them to process the message defensively. However, we did not explicitly assess defensive processing; it is thus impossible to infer from our data to what extent differences in levels of defensive processing were responsible for the effect. Further research is needed to investigate the role that defensive processing plays in message framing effects. Second, the experimental setting of this study might have created a demand effect. The experiment leaders were instructed to assure participants that their answers would be confidential. However, since participants filled in the questionnaire in a somewhat crowded place, it can not be ruled out that participants reported levels of precautionary motivation that were socially desirable. Third, because we measured selfefficacy in the present study, we can not rule out that self-efficacy was confounded with other variables, like, for instance, self-esteem. Future studies on the role of self-efficacy in framing effects should try to manipulate self-efficacy to be able to rule out alternative explanations. Fourth, our study did not include a long-term follow-up. This makes it impossible to assess behavioural effects. Future research should investigate the long-term behavioural effects of framing in people with high and low levels of self-efficacy.

In sum, our findings underline the importance of self-efficacy in persuasion. They suggest that self-efficacy, besides from being a major determinant of motivation to engage in healthy behaviour (De Vries \& Backbier, 1994; Schwarzer, 1992; Strecher et al., 1986) can also influence the differential persuasive effects of gain and loss-framed health promoting messages. 


\title{
Chapter 6: Self-Efficacy Moderates Message- Framing Effects: The Case of Skin-Cancer De- tection $^{11}$
}

\begin{abstract}
Health-promoting messages can be framed in terms of the gains associated with healthy behaviour or the losses associated with unhealthy behaviour. Studies show inconsistent results as to which type of framing is more effective. In this study we examined the influence of self-efficacy to perform skin selfexamination on the effects of gain- and loss-framed skin-cancer detection messages among 124 university students. For participants with high self-efficacy, a loss-framed message resulted in a higher intention to perform skin selfexamination than a gain-framed message. For participants with low self-efficacy, there were no differences in intention between the gain- and loss-framed message conditions. Our results suggest that self-efficacy levels play an important role in message-framing effects. For health communication strategies promoting the active detection of skin-cancer symptoms, messages stressing losses may be more effective than messages stressing gains, but only in persons with high selfefficacy. In addition, our results suggest that health promoting messages can be framed to match recipients' self-efficacy levels.
\end{abstract}

\section{Introduction}

Skin cancer incidence is increasing rapidly and constitutes a significant health concern. For 2008, the incidence of melanoma, the most dangerous form of skin cancer, was estimated at 62,480 in the United States, with mortality at 8,420 (American Cancer Society, 2008a). Each year, approximately 25,000 individuals are diagnosed with skin cancer in the Netherlands (Dutch Cancer Society, 2006). In light of these figures, early detection of skin-cancer symptoms is of great importance. If cancer is diagnosed early, the patient has a greater chance of successful treatment (American Cancer Society, 2008b). Skin self-examination refers to the act of inspecting one's skin with the aim of detecting possible skincancer related symptoms. Especially when performed frequently, preferably once a month, it can be an effective way to detect skin cancer at an early stage (American Cancer Society, 2008b). The present study investigated whether the persuasiveness of communications promoting skin self-examination can be improved by message framing.

Persuasive health messages can be framed in terms of the benefits of engaging in healthy behaviour (gain frame), or in terms of the costs of failing to 
engage in healthy behaviour (loss frame). Empirical studies have shown that gain- and loss-framed messages can have different effects on individuals' selfprotective actions or motivation to perform healthy behaviours, even when the persuasive information in gain- and loss-framed health messages is factually equivalent (Rothman, Bartels, Wlaschin, \& Salovey, 2006; Rothman \& Salovey, 1997). However, studies also show inconsistent results with regard to which type of framing is more persuasive (for meta-analyses see Kuhberger, 1998; O'Keefe \& Jensen, 2006; 2007). One influential approach to the study of message framing has been to focus on the function of the recommended behaviour, making a distinction between behaviours that serve to prevent an illness (like exercising or quitting smoking) and behaviours that serve to detect an illness (like skin selfexamination or obtaining a mammography). According to Rothman and Salovey (1997), people perceive disease-prevention behaviours as relatively safe, because they minimize the chance of falling ill. In contrast, people perceive diseasedetection behaviours as inherently risky because they entail the possibility of finding out that one is ill. Drawing from Prospect Theory (Kahneman, 2003; Kahneman \& Tversky, 1984), Rothman and Salovey (1997) go on to suggest that gain-framed information is more persuasive when advocating disease-prevention behaviours, because gain-framed information makes people risk-avers and thus more likely to engage in relatively safe disease-prevention behaviours. In contrast, they suggest that loss-framed information is more persuasive for diseasedetection behaviours, because loss-framed information makes people willing to take risks and thus more likely to engage in relatively risky disease-prevention behaviours. A recent meta-analysis showed, however, that for behaviours serving to detect an illness, gain- and loss-framed messages were not differentially persuasive (O'Keefe \& Jensen, 2006). Thus, in contrast to Rothman and Salovey's reasoning, and despite an impressive body of literature, it is currently unclear how professionals should frame messages promoting active cancer detection in general and skin self-examination in particular.

To explore the reasons for these inconsistent results, research is needed that investigates under which circumstances gain- or loss-framed information is more persuasive. Research investigating possible moderating variables that can explain differences in the effects of gain- and loss-framed messages is indispensable to deepening our understanding of message framing's effects on persuasion. The aim of the present study, therefore, was to investigate whether selfefficacy can moderate the effects of framed health-promoting messages. The concept of self-efficacy is derived from social cognitive theory (Bandura, 1986) and refers to a person's belief in her abilities to perform a given action. Drawing from fear-appeal literature, we hypothesized that loss-framed messages might be more persuasive than gain-framed communications, but only for recipients with high self-efficacy. 
Loss-framed messages have been found to evoke a greater sense of threat than gain-framed messages (Cox \& Cox, 2001; Shen \& Dillard, 2007). Fear appeal research suggests that this greater sense of threat might increase persuasiveness, but only if recipients feel capable of averting the threat by performing the recommended action (Rogers, 1983; Stephenson \& Witte, 1998; Witte, 1992, 1994; for an overview, see Ruiter, Abraham, \& Kok, 2001). If recipients have low self-efficacy levels, however, this greater sense of threat may result in less message acceptance due to defensive avoidance and message derogation processes (cf. Witte, 1992). The latter theoretical assumption explains why, in some cases, loss-framed messages are more persuasive than gain-framed messages (e.g. Banks et al., 1995; Cherubini, Rumiati, Rossi, Nigro, \& Calabro, 2005; Meyerowitz \& Chaiken, 1987), whereas in other cases they are not (e.g. Van Assema, Martens, Ruiter, \& Brug, 2001; Cox, Cox, \& Zimet, 2006).

In a recent study (Van 't Riet, Ruiter, Werrij, \& De Vries, 2008) these assumptions were tested using framed anti-smoking messages targeted at current smokers. The results supported the hypothesis and demonstrated that selfefficacy to quit smoking moderated the influence of gain- and loss-framed antismoking messages. In particular, a loss-framed communication was more persuasive for participants with high self-efficacy to quit smoking than a gainframed communication or no communication. For participants with low selfefficacy to quit smoking, there was no difference in persuasiveness between the gain-framed, loss-framed and no-communication control conditions. In the present study, we aimed to investigate whether these previous findings can be extended to another health domain (skin self-examination). As outlined above, several scholars have distinguished between behaviours that serve to prevent illness and those that serve to detect illness (e.g., Rothman \& Salovey, 1997). In fact, it has been argued that message framing has different effects on prevention- versus detection-behaviours. The present study aimed to investigate whether the moderating role of self-efficacy, found in a study of framed messages advocating a prevention behaviour (smoking cessation) could be replicated using framed messages advocating a detection-behaviour (skin self-examination).

In short, an influential approach to the study of message framing posits that for the promotion of disease-detection behaviours, loss-framed information is more persuasive than gain-framed information (Rothman \& Salovey, 1997). Recent meta-analytic findings, however, shed doubt on these expectations. For this reason, we do not expect to find an overall advantage of loss-framed information over gain-framed information. Instead, we are proposing a new framework based on social cognitive theory (Bandura, 1986) and fear-appeal research (e.g., Ruiter et al., 2001), hypothesizing that self-efficacy can moderate the impact of gain- and loss-framed messages. In the present study we hypothesised that loss-framed messages would be more persuasive than gain-framed messages, but only for participants with high self-efficacy. For participants with low 
self-efficacy, we predicted that loss-framed messages would not be more persuasive than gain-framed messages, because the greater sense of threat conveyed by the loss-framed as compared to the gain-framed message would make them react defensively. The experiment had two main outcome measures. First, information acceptance was assessed as a measure of defensive reactions to the healthpromoting information. Although there is no current 'gold standard' for the measurement of defensive processing, a meta-analysis by Good and Abraham (2007) indicated that measuring information acceptance is a valid way to assess defensive reactions. Intention to engage in monthly skin self-examination was assessed as a measure of persuasion.

\section{Method}

\section{Participants}

Students at Maastricht University listed in a database of voluntary research participants were invited by email to take part in the experiment. Out of a total of 768 eligible participants in the database, 124 students enrolled in the experiment in exchange for $€ 5$. Most were female (107 vs 17 males), and age ranged from 18 to 26 years, with a mean age of 20.7 years $(S D=1.6)$.

\section{Design and procedure}

The present study used a one-factorial (frame: gain frame vs loss frame) between-participants design. ${ }^{12}$ There were two main outcome measures: information acceptance assessed as a measure of defensive reactions to the healthpromoting information, and intention to engage in monthly skin self-examination as a measure of persuasion.

Participants were scheduled to visit the laboratory, and were seated in individual booths. We told participants that they were about to participate in a study to test health education materials that we had designed for use 'on the internet'. First, we assessed participants' baseline self-efficacy levels to perform skin self-examination. Next, we provided them with a persuasive communication about skin self-examination, which was either gain- or loss-framed. Participants were randomized into the gain- and loss-framed conditions by means of a random-number generator, resulting in 61 participants in the gain-framed condition and 63 participants in the loss-framed condition. After reading the persuasive communication, participants completed the dependent measures, then were debriefed and received their fee.

\section{Framing manipulation}

For the present study, we designed short communications of approximately 200 words, stressing either the benefits of performing skin self- 
examination (gain frame), or the costs of not performing skin self-examination (loss frame). The full texts are available in Appendix C. For the purpose of the present study, it was especially important that the loss-framed communication was perceived as more threatening than the gain-framed communication. To ensure that this was the case, we tested the effects of the gain- and loss-framed communications on perceived threat in a pretest. Conducting manipulation checks in separate pretests may be preferable to conducting them in the main experiment that examines the dependent variables, because manipulation checks placed before the dependent variables might influence the latter, whereas manipulation checks placed after the dependent variables might fail to detect stimulus effects (Perdue \& Summers, 1986; see also Cox et al., 2006). The results of a pretest among 41 Maastricht University students (36 females; mean age $=20.8$ years, $S D=1.8$ ), revealed that, in line with previous research (Cox et al., 2001; Shen \& Dillard, 2007), the loss-framed communication was perceived as significantly more threatening than the gain-framed communication as assessed by one item asking participants to indicate the extent to which they thought the information in the communication was threatening on a 7-point scale $(1=$ not threatening at all; $7=$ very threatening), $B=1.35, t(38)=3.51, p=.001, s r=.50$.

\section{Measures}

Base-line self-efficacy. To measure participants' baseline self-efficacy to perform skin self-examination, one item, using a 7-point scale, asked participants: 'If you would try to perform skin self-examination once a month, would you be able to do this?' ( $1=$ no, definitely not; 7 = yes, definitely).

Information acceptance. Five items assessed participants' acceptance of the information. Participants rated the information on 7-point semantic differentials as (1) very relevant to (7) not at all relevant; (1) very interesting to (7) not at all interesting; (1) very exaggerated to (7) not at all exaggerated; (1) very objective to (7) not at all objective. In addition, perceived manipulation was measured: participants rated their agreement with the statement: 'The information I received tried to manipulate my feelings' on a 7-point scale $(1=I$ definitely agree; $7=I$ definitely do not agree). The scores on the relevant, interesting and objective items were mirrored and the five items combined to form an average information acceptance score (Cronbach's $\alpha=.64$ ).

Intention. Four items assessed participants' intention to perform skin self-examination. Participants were asked to indicate whether they agreed with the statements ' $I$ intend to examine my skin once a month in the coming six months' and 'I am thinking about examining my skin once a month', both on 7point scales $(1=$ I definitely do not agree; $7=$ I definitely agree $)$. They were also asked to indicate how likely it was that they would examine their skin once a month in the coming six months, and how likely it was that they would examine 
their skin once a month in the future, both on a 7-point scale $(1=$ very unlikely; 7 $=$ very likely). From these four items an average score was calculated $(\alpha=.87)$.

\section{Data analysis}

Regression analyses tested the main effects of frame (coded as $0=$ gain frame, 1 = loss frame), baseline self-efficacy and the Frame X Self-Efficacy interaction term. To ensure that multicollinearity did not affect the results, individual scores on the self-efficacy measure were centred by subtracting the mean from each score (see Aiken \& West, 1991). In case of a significant interaction effect, simple slope analyses were conducted to investigate the nature of the interaction (for a more elaborate description of analysing interactions in multiple regression, see Aiken \& West, 1991).

\section{Results}

\section{Information acceptance}

Our analyses revealed that participants with higher self-efficacy showed higher ratings of information acceptance, $B=.25, t(121)=3.73, p<.001$, $s r=$ .32. In addition, participants had lower ratings of information acceptance after having received loss-framed as opposed to gain-framed information, although this difference was only marginally significant, $B=-.27, t(121)=-1.86, p=.07$, $s r=.16$. The interaction between frame and baseline self-efficacy did not contribute significantly to the prediction of information acceptance, $B=.16, t(120)=$ $1.16, p=.25, s r=.10$.

\section{Intention}

Regression analyses with intention as the dependent variable revealed that the interaction between frame and baseline self-efficacy contributed significantly to the prediction of intention, $B=.62, t(120)=2.97, p<.01, s r=.25$. Next, simple slope analyses were performed to analyse the main effect of frame for participants with high baseline self-efficacy (one $S D$ above the mean of the centred baseline self-efficacy score) and for those with low baseline self-efficacy (one $S D$ below the mean of the centred baseline self-efficacy score). Supporting our hypothesis, simple slope analyses showed that there was a significant effect of frame on intention for participants with high self-efficacy, such that lossframed communications resulted in a higher intention than gain-framed communications, $B=.88, t(120)=2.88, p<.01, s r=.24$. For participants with low selfefficacy, there was no significant difference between the gain- and loss-framed condition, $B=-.49, t(120)=-1.50, p=.14, s r=-.13$ (see Figure 6). 
Figure 6 Regression slopes arising from the relationship between baseline self-efficacy and intention for participants who received a gain-framed communication versus those who received a loss-framed communication

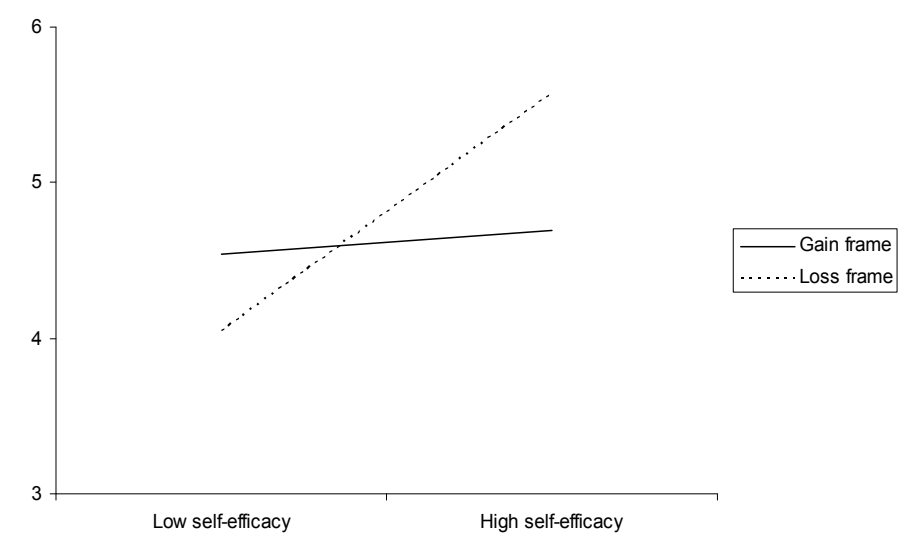

\section{Discussion}

The United States and other western countries have recently seen a rise in the incidence of skin cancer (American Cancer Society, 2008b). Given the fact that early diagnosis of skin cancer can enhance the patient's chance of successful treatment, it is becoming increasingly important to inform the public about the benefits of early detection of cancer-related symptoms. Unfortunately, recent research shows that this can be a very challenging task (Ruiter, De Nooijer, Van Breukelen, Ockhuysen-Vermey, \& De Vries, 2008). The present study investigated whether the persuasiveness of

communications promoting skin self-examination can be improved by message framing. In particular, we aimed to investigate under which circumstances gainor loss-framed messages promoting skin self-examination are more persuasive.

We hypothesised that loss-framed messages would be more persuasive than gain-framed messages, but only for participants with high self-efficacy to engage in monthly skin self-examination. When self-efficacy is low, threatening health-promoting messages might be processed defensively. The negative information in loss-framed messages may therefore be avoided or dismissed, and as a result, loss-framed messages might lose their advantage over gain-framed messages. Our results supported this hypothesis.

The analyses of information acceptance, our measure of defensive reactions to the information, first revealed that participants with low self-efficacy were less likely to accept information about skin self-examination than those 
with high self-efficacy, suggesting that they engaged in greater levels of defensive processing than participants with high self-efficacy. Second, the loss-framed communication resulted in lower levels of information acceptance than the gainframed communication, indicating that the loss-framed communication cued more defensive reactions. The latter difference, however, was only marginally significant. These results suggest that the combination of having low selfefficacy and receiving a loss-framed communication might render recipients of health promoting information particularly likely to process this information defensively. The interaction between message frame and self-efficacy, however, was not significant.

The analyses of intention showed that participants with high baseline self-efficacy to perform skin self-examination who read a loss-framed communication were more motivated to perform skin self-examination than those who read a gain-framed communication. For participants with low baseline selfefficacy to perform skin self-examination, we found no differences in intention between the gain- and loss-framed conditions.

The present study replicated the findings of a previous study which found that self-efficacy to quit smoking moderated the effects of gain- and lossframed anti-smoking communications among adult smokers (Van 't Riet et al., 2008). Our results suggest that this effect can be generalised across populations and across health domains. In organisational psychological research, studies have shown that people with high levels of self-efficacy set higher goals (Locke \& Latham, 2002) and display greater task persistence than those with low selfefficacy (Bandura \& Cervone, 1986). In health behaviour research, self-efficacy has been found to be a major determinant of motivation to engage in healthy behaviour (De Vries \& Backbier, 1994; Schwarzer, 1992; Strecher et al., 1986). Our results show that self-efficacy can also influence the differential effects of gain- and loss-framed health-promoting messages on intention to engage in healthy behaviour.

In contrast with previous research that suggests that loss-framed information is more persuasive than gain-framed information for disease-detection behaviours (e.g., Rothman \& Salovey, 1997) but in line with recent metaanalytic findings (O'Keefe \& Jensen, 2006), we did not find an overall advantage of loss-framed information. We propose that the absence of an overall framing effect in the present study once again highlights the importance of investigating possible moderators of message-framing effects. Our results suggest that self-efficacy qualifies as an important moderator. More generally, our findings raise questions as to whether the postulates of Prospect Theory can be effectively applied to message framing. In response to similar questions raised by O'Keefe \& Jensen (2007), Latimer and colleagues (2007) have argued that Prospect Theory could still provide an adequate framework for studying message framing, but that researchers should focus less on whether the recommended behaviour serves 
to prevent or detect illness, and more on the way the recipient perceives the behaviour. According to this reasoning, loss-framed information might be more effective to the extent that the recipient perceives the recommended behaviour as risky. Future studies should investigate this hypothesis.

The present study was subject to certain limitations. First, our sample was predominantly female. Future studies should investigate whether the same results can be obtained by a more balanced or a male sample. Second, we operationalized defensive reactions as measures of participants' information acceptance. Our assessment of information acceptance, however, had less than perfect internal consistency $(\alpha=.64)$. Also, there are various measures that can be used to assess defensive reactions, such as avoidance and fatalism (see for instance Rippetoe \& Rogers, 1987) or more direct assessments, like the number of counter-arguments generated by recipients (Keller \& Block, 1999) or reading time (Reed \& Aspinwall, 1998). Although the results of a recent meta-analysis indicate that information acceptance is a reliable measure of defensive reactions (Good \& Abraham, 2007), by using information acceptance scores only we may have overlooked other important defensive processes. Third, we measured selfefficacy to perform skin self-examination. Evidence that a self-efficacy manipulation could influence the effects of framed communications, however, would allow us to draw firmer conclusions about the causality of the effect. Given the correlational nature of our design, we cannot completely rule out that the effect of self-efficacy may have been due to the influence of other confounding variables, such as self-esteem or optimism. Also, we used a single-item assessment of self-efficacy. Future studies investigating the role of self-efficacy in messageframing effects should employ more refined measures to assess self-efficacy. Finally, our study did not include a long-term follow-up, making it impossible to assess behavioural effects. Future research should investigate the long-term behavioural effects of framing and self-efficacy or should use proximal measures of behaviour, like willingness to register for a course in skin self-examination (cf. Ruiter, Verplanken, De Cremer, \& Kok, 2001) or willingness to fill in a coupon to receive additional information (cf. Detweiler, Bedell, Salovey, Pronin, Rothman, 1999).

Future research that deals with these limitations might provide more insight in the influence of self-efficacy on message framing effects. For instance, it could be worthwhile to identify sub-populations in which the moderating effect of self-efficacy is particularly likely to occur. For recipients that have a personal or family history of skin cancer, for example, messages about the negative consequences of not performing skin self-examination might be particularly threatening, making it more likely that self-efficacy will influence their reactions towards these messages. In addition, future research should investigate whether the effect can be replicated with other disease-detection behaviours, such as breast self-examination or obtaining a mammography. 


\section{Chapter 6}

Despite these limitations, our findings underline the importance of selfefficacy in persuasion. They suggest that self-efficacy, besides being a major determinant of motivation to engage in healthy behaviour (De Vries \& Backbier, 1994; Schwarzer, 1992; Strecher et al., 1986), can also influence the differential persuasive effects of gain- and loss-framed health-promoting messages. In addition, the results of this study suggest that health education interventions could take recipients' self-efficacy into account. In line with the growing practice to tailor health-education interventions to recipients' individual characteristics (Dijkstra \& De Vries, 1999; Rimer \& Kreuter, 2006; Ruiter, Kessels, Jansma, \& Brug, 2006), the frame of a health-promoting communication could be tailored to recipients' self-efficacy levels. 


\title{
Chapter 7: Examining the Influence of Self- Efficacy on Message-Framing Effects: Reduc- ing Salt Consumption in the General Popula- tion $^{13}$
}

\begin{abstract}
Health-promoting messages can be framed in terms of the gains associated with healthy behaviour or the losses associated with unhealthy behaviour. Studies show inconsistent results as to which type of framing is more effective. In this study we investigated whether the effects of gain- and loss-framed messages promoting a low-salt diet would be moderated by participants' selfefficacy to decrease salt-intake. We hypothesized that loss-framed messages would more effectively decrease salt-intake than gain-framed messages, but only when participants had a high self-efficacy. A total of 575 adults, recruited from an Internet-panel, took part in the study. Half of the participants received selfefficacy enhancing information whereas the other half received no such information. After this self-efficacy manipulation half of the participants received a gainframed, and half of the participants received a loss-framed message promoting a low-salt diet. Salt consumption was assessed as the main outcome variable at a 3-week follow-up. The results supported our hypothesis. The effect of the interaction between self-efficacy and framing on salt consumption was not mediated by measures of information acceptance and intention to reduce salt-intake. Our results suggest that messages stressing losses may be more effective than messages stressing gains, but only in persons with high self-efficacy.
\end{abstract}

\section{Introduction}

High salt intake is a major risk factor for the development of hypertension (Blaustein, Zhang, Chen, \& Hamilton, 2006), which in turn is a key determinant of cardiovascular disease (He \& Whelton, 1999). Because cardiovascular disease accounts for approximately one-third of all deaths world-wide, the World Health Organization has recommended that individuals limit their intake of dietary sodium from all sources to less than 5 grams per day (WHO, 2003). In the Netherlands, average salt-intake is much higher, reaching approximately 8.8 grams a day (Van den Hooven, Fransen, Jansen, \& Ocké, 2007). A reduction in the total amount of consumed salt will reduce the prevalence of hypertension and of cardiovascular disease (Cook et al., 2007; Van den Hooven et al., 2007). Since it is imperative to develop effective public-health messages that can encourage people to reduce their salt-intake, the present study investigated whether the per- 
suasiveness of communications promoting a low-salt diet can be improved by message framing.

Health messages can be framed in terms of the benefits of engaging in healthy behaviour (gain frame), or in terms of the costs of failing to engage in healthy behaviour (loss frame). Empirical studies have shown that gain- and loss-framed messages can have different effects on individuals' self-protective actions or motivation to perform healthy behaviours, even when the persuasive information in gain- and loss-framed health messages is factually equivalent (Rothman, Bartels, Wlaschin, \& Salovey, 2006; Rothman \& Salovey, 1997). However, studies also show inconsistent results with regard to which type of framing is more persuasive (for meta-analyses see Kuhberger, 1998; O'Keefe \& Jensen, 2006; 2007). One influential approach to the study of message framing has been to focus on the function of the recommended behaviour, making a distinction between behaviours that serve to prevent an illness (like exercising or quitting smoking) and behaviours that serve to detect an illness (like skin selfexamination or obtaining a mammography). According to Rothman and Salovey (1997), people perceive disease-prevention behaviours as relatively safe because they minimize the chance of falling ill. In contrast, people perceive diseasedetection behaviours as inherently risky because they entail the possibility of finding out that one is ill. Drawing from Prospect Theory (Kahneman, 2003; Kahneman \& Tversky, 1984), Rothman and Salovey (1997) go on to suggest that gain-framed information is more persuasive when advocating disease-prevention behaviours because gain-framed information makes people risk-avers and thus more likely to engage in relatively safe disease-prevention behaviours. In contrast, they suggest that loss-framed information is more persuasive for diseasedetection behaviours, because loss-framed information makes people willing to take risks and thus more likely to engage in relatively risky disease-detection behaviours. A recent meta-analysis showed, however, that for behaviours serving to detect an illness, gain- and loss-framed messages were not differentially persuasive (O'Keefe \& Jensen, 2006). For prevention behaviours a small advantage of gain-framed information was found, but this effect only occurred in a limited amount of studies on dental health (O'Keefe \& Jensen, 2007). Thus, in contrast to Rothman and Salovey's reasoning, and despite an impressive body of literature it is currently unclear how professionals should frame health-promoting messages.

To explore the reasons for these unsatisfying results, research is needed that investigates under which circumstances gain- or loss-framed information is more persuasive. Research investigating possible moderating variables that can explain differences in the effects of gain- and loss-framed messages is indispensable to deepening our understanding of message-framing effects on persuasion. The aim of the present study, therefore, was to investigate whether self-efficacy can moderate the effects of framed health-promoting messages. The concept of 
self-efficacy is derived from social cognitive theory (Bandura, 1986) and refers to a person's belief in her abilities to perform a given action. Drawing from fearappeal literature, we hypothesized that loss-framed messages might be more persuasive than gain-framed communications but only for recipients with high selfefficacy.

Loss-framed messages have been found to evoke a greater sense of threat than gain-framed messages (Cox, Cox, \& Zimet, 2006; Shen \& Dillard, 2007; Van 't Riet, Ruiter, Werrij, \& De Vries, in press). Fear appeal research suggests that this greater sense of threat might increase persuasiveness, but only if recipients feel capable of averting the threat by performing the recommended action (Rogers, 1983; Stephenson \& Witte, 1998; Witte, 1992; 1994; for an overview see Ruiter, Abraham, \& Kok, 2001). If recipients have low selfefficacy levels, however, this greater sense of threat may result in less message acceptance due to defensive avoidance and message derogation processes (cf. Witte, 1992).

In two recent studies these assumptions were tested using framed antismoking messages (Van 't Riet et al., 2008) and messages promoting breast selfexamination (Van 't Riet et al., in press). The results supported the hypothesis and demonstrated that self-efficacy moderated the influence of gain- and lossframed messages on intentions to perform healthy behaviour. In particular, lossframed communications were more persuasive than gain-framed communications for participants with high self-efficacy. For participants with low selfefficacy, there was no difference in persuasiveness between gain- and lossframed communications. These two studies, however, suffered from two important limitations. First, they both used self-report measures of self-efficacy, whereas evidence that a self-efficacy manipulation could moderate the effects of framed communications would allow us to draw firmer conclusions about the causality of the effect. Indeed, these studies cannot rule out that the effect of selfefficacy was due to the influence of confounding variables, such as self-esteem or optimism. Second, neither of the two studies included a long-term follow-up, making it impossible to assess behavioural effects. From a public health pointof-view it is important to investigate whether framing and self-efficacy can interact to produce effects on actual health behaviour.

In the present study, we aimed to investigate whether these previous findings could be replicated, making several adjustments in the study design to account for the limitations of the earlier studies. First, to provide a more robust test of our hypothesis and to be able to rule out alternative explanations, we used a self-efficacy manipulation instead of a self-efficacy assessment. Second, we included a three-week follow-up assessment to investigate whether self-efficacy could moderate the effects of framing on behaviour (in this case salt consumption). 


\section{The Present Study}

Recently, a new theoretical framework to account for the effects of gain- and loss-framed messages has been proposed, based on Social Cognitive Theory (Bandura, 1986) and fear-appeal research (e.g., Ruiter, Abraham, \& Kok, 2001), hypothesizing that self-efficacy can moderate the impact of gain- and loss-framed messages (Van 't Riet et al., 2008; in press). In the present study, this framework is tested using a self-efficacy manipulation, framed messages promoting a low-salt diet and a three-week follow-up measure of behaviour. Because for the purpose of the study it was especially important that the lossframed communication was perceived as more threatening than the gain-framed communication, we tested the effects of the gain- and loss-framed communications on perceived threat and negative affect in a pretest. Conducting manipulation checks in separate pretests may be preferable to conducting them in the main experiment that examines the dependent variables (Perdue \& Summers, 1986), especially when the manipulation check or dependent measure is meant to assess affectively laden constructs (see also Cox, Cox, \& Zimet, 2006). The rationale for this is that manipulation checks placed before the dependent variables might influence the latter, whereas manipulation checks placed after the dependent variables might fail to detect stimulus effects.

In the main experiment we tested the hypothesis that loss-framed messages would be more persuasive than gain-framed messages, but only for participants with high self-efficacy. For participants with low self-efficacy we predicted that loss-framed messages would not be more persuasive than gain-framed messages because the greater sense of threat conveyed by the loss-framed as compared to the gain-framed message would make them react defensively. The main experiment had three main outcome measures. Information acceptance was assessed as a measure of defensive reactions to the health-promoting information. Although there is no current 'gold standard' for the measurement of defensive processing, a recent meta-analysis (Good and Abraham, 2007) indicated that measuring information acceptance is a valid way to assess defensive reactions. Intention to reduce salt-intake was assessed at an immediate follow-up and saltintake was assessed at a three-week follow-up.

\section{Pretest}

\section{Method}

Procedure and Design. Potential participants were recruited from a Dutch Internet research panel. The only inclusion criterion was that participants had to be at least 18 years of age. In total, 390 participants took part in the study. Participants received a persuasive communication encouraging them to reduce 
their salt consumption and afterwards filled out the manipulation check measures.

Participants. The sample consisted of $51.0 \%$ women (199 vs 191 males), and age ranged from 18 to 83 years, with a mean age of 49.0 years $(S D=$ 15.6). In total, 142 participants had a high education, 134 had a medium education, and 114 had a low education. In the complex schooling system in the Netherlands, a low education level refers to primary or basic vocational school, a medium education level refers to secondary vocational school or high school, and a high education level refers to advanced vocational school or university.

Framing manipulation. The persuasive communication either stressed the advantages of a low-salt diet or the disadvantages of a high-salt diet. The full texts are available in Appendix E.

Manipulation checks. To measure perceived threat one item assessed the extent to which participants thought the information was threatening ( $1=n o t$ at all threatening; $7=$ very threatening), one item assessed the extent to which participants thought the information was frightening $(1=$ not at all frightening; 7 = very frightening), and one item assessed whether the information was reassuring ( 1 = not at all reassuring; 7 = very reassuring). These items were used separately in the analysis. Furthermore, eight items assessed negative affect, asking participants to indicate the extent to which the information made them feel (1) very tense to (7) not at all tense; (1) very uneasy to (7) not at all uneasy; (1) very worried to (7) not at all worried; (1) very afraid to (7) not at all afraid; (1) very disappointed to (7) not at all disappointed; (1) very sad to (7) not at all sad; (1) very depressed to (7) not at all depressed. These items were reversed to create an average negative affect score $(\alpha=.96)$.

\section{Results}

A series of t-tests revealed that the loss-framed communication was perceived as more threatening than the gain-framed communication $(M=4.1$ vs. $\mathrm{M}=3.1 ; t=6.11, p<.001)$. Furthermore, the loss-framed communication was perceived as more frightening $(\mathrm{M}=4.0$ vs. $\mathrm{M}=3.0 ; t=6.51, p<.001)$, less reassuring $(\mathrm{M}=3.4$ vs. $\mathrm{M}=4.4 ; t=7.22, p<.001)$, and resulted in higher levels of negative affect $(\mathrm{M}=2.9$ vs. $\mathrm{M}=2.4 ; t=3.82, p<.001)$.

\section{Discussion}

The hypothesis that self-efficacy moderates message-framing effects depends on the assumption that loss-framed messages are perceived as more threatening than gain-framed messages. The results of the pretest showed that for the messages that were designed in the present study this was indeed the case. In the main experiment we now used these messages to test our main hypothesis. 


\section{Main Experiment}

Method

Procedure and Design. Potential participants were recruited from a Dutch Internet research panel. Out of a total of approximately 19,000 people in the Internet panel, 900 adults were invited to participate in the study. The panel is representative of the total Dutch population with regards to age, gender, education and region. The only inclusion criterion was that participants had to be at least 18 years of age. In total 575 participants accepted this invitation and took part in the study. Following acceptance of the invitation, they immediately accessed the first part of the study. This consisted of a baseline questionnaire assessing salt consumption. Next, participants received a communication encouraging them to reduce their salt intake. In the high self-efficacy condition, the communication emphasized that it is easy to reduce salt intake and stressed various possibilities to reduce salt-intake (e.g., 'Instead of salt, you can put vinegar on your vegetables and salads'). In the low self-efficacy condition, participants did not receive this self-efficacy enhancing information. The full text of the selfefficacy manipulation is available in Appendix F. Next, participants received a persuasive communication about salt-intake, which was either gain- or lossframed. The gain- and loss-framed messages used in the main experiment were the same messages that were used in the pretest (Appendix E).

Participants were randomized into the four conditions of the 2 (selfefficacy) x 2 (framing) between-participants design by means of a randomnumber generator, resulting in 145 participants in the low self-efficacy / gainframe condition, 146 participants in the low self-efficacy / loss-frame condition, 143 participants in the high self-efficacy / gain-frame condition, and 141 participants in the high self-efficacy / loss-frame condition.

After participants received the persuasive communication, they received the immediate post-test questionnaire, which included a manipulation check of the self-efficacy manipulation, and measures of information acceptance and intention. Three weeks after the first measurement, they were sent an email, inviting them to participate in the follow-up measurement, which assessed salt-intake. No reminder was send in case of non-response. In total, 516 participants took part in the follow-up measurement ( $89.7 \%$ response rate).

Participants. Most participants were female (306 vs 269 males), and age ranged from 19 to 88 years, with a mean age of 49.3 years $(S D=15.6)$. In total, 166 participants had a high education, 240 had a medium education, and 169 had a low education.

Baseline measures. To measure participants' baseline salt-intake, an index score of salt consumption was created (this is in line with previous research; cf. Smerecnik, Mesters, De Vries, \& De Vries, in preparation) four items assessed various eating habits that could influence the total intake of sodium. 
First, participants were asked: 'Do you generally eat salty meals?' $(1=$ no, never; $5=$ yes, very often). Next, participants were asked to indicate whether they generally consumed a lot of salt $(1=n o$, not at all; $5=y e s$, very $)$, and whether they often added salt during cooking $(1=$ no, never; $5=$ yes, always $)$. Next, participants were asked on how many of the previous seven days they had added salt to their meal. These four items were added up to create a salt-consumption index.

Post-test measures. To check whether the manipulation of self-efficacy had been successful, eight manipulation checks assessed participants' post-test self-efficacy to reduce their salt-intake. First, five items asked participants to indicate on a 7-point scale whether they agreed with the statements: 'Eating less salt is easy', 'There are plenty of alternatives for salt', 'I know how to season my dinner without salt', 'If I would attempt to eat less salt, I would know which products to use to give flavour to my dinner', 'I can cook a nice meal without salt if I want to' ( 1 = I totally disagree; $7=$ I totally agree $)$. Next, three items asked participants to indicate whether they thought they would succeed to eat less salt, whether they thought they would succeed to eat less salt in the coming week, and whether they thought they would succeed to eat less salt in the coming year, if they would try this $(1=$ No, would certainly not succeed; $7=$ Yes, would certainly succeed). From these items, an average self-efficacy score was created $(\alpha=.91)$.

Eight items assessed information acceptance by asking participants to indicate the extent to which they thought the information was true $(1=$ Very true; $7=$ Not at all true $)$, objective $(1=$ Very objective; $7=$ Not at all objective $)$, convincing $(1=$ Very convincing; $7=$ Not at all convincing $)$, relevant $(1=$ Very relevant; $7=$ Not at all relevant $)$, believable $(1=$ Very believable $7=$ Not at all believable $)$, useful $(1=$ Very useful; $7=$ Not at all useful $)$, and interesting $(1=$ Very interesting; $7=$ Not at all interesting). Furthermore, one item asked participants to indicate the extent to which participants agreed with the information $(1=I$ totally agree; $7=I$ totally disagree). After we reversed the scores of all items, the scores on the eight items were averaged to create an information acceptance score $(\alpha=.94)$.

Six items were used to assess intention to eat less salt, using a 7-point scale. One item asked participants 'how likely is it that you will eat less salt in the future?' ( 1 = Very likely; 7 = Very unlikely). One item asked participants: 'how likely is it that you will eat less salt in the coming week?' ( 1 = Very likely; $7=$ Very unlikely). Two items asked participants whether they planned to eat less salt in the future and in the coming week. One item asked participants whether they considered eating less salt in the future and one item asked participants whether they were sure that the would eat less salt in the coming week $(1=\mathrm{Cer}$ tainly; $7=$ Certainly not). Scores on the intention items were reversed and an average intention score was calculated $(\alpha=.96)$. 
Follow-up. To assess behaviour at the three-week follow-up measurement, the same four items were used as in the baseline questionnaire.

Data analysis. Regression analyses tested the main effects of selfefficacy (coded as $0=$ low self-efficacy, $1=$ high self-efficacy), frame (coded as 0 = gain frame, $1=$ loss frame), and the frame by self-efficacy interaction term on the self-efficacy manipulation checks, information acceptance, intention, and behaviour. In case of a significant interaction effect, simple slope analyses were conducted to investigate the nature of the interaction (for a more elaborate description of analysing interactions in multiple regression, see Aiken \& West, 1991).

\section{Results}

Randomization check. To test whether randomization had been successful, one-way ANOVAs and chi-square analyses were performed. The analyses showed that there were no significant differences between conditions with regards to age, gender, education and baseline salt-consumption, $p \mathrm{~s}>.65$.

Attrition analyses. Logistic regression analyses were performed with dropout as the dependent variable to investigate whether frame, self-efficacy, the interaction between frame and self-efficacy, salt consumption at baseline and education predicted dropout from loss to follow-up. Participants in the lossframe condition were less likely to drop-out than participants in the gain-frame condition $(5.9 \%$ of participants were lost to follow-up in the loss-frame condition versus $14.6 \%$ in the gain-frame condition; $\mathrm{OR}=.36$, Wald $=11.28, p=.001$ ). The other variables did not have significant contributions to the prediction of dropout, $p \mathrm{~s}>.14$.

Self-efficacy manipulation check. The results of the linear regression analyses showed that, in line with expectations, participants in the high selfefficacy condition had higher post-test self-efficacy than participants in the low self-efficacy condition, $B=.28, t(572)=2.84, p<.01$. There were no significant effects of frame or the frame by self-efficacy interaction term, $p \mathrm{~s}>.43$.

Information acceptance. Contrary to our hypotheses, the analyses revealed no effects of self-efficacy condition, frame, or the frame by self-efficacy interaction term on information acceptance, $p \mathrm{~s}>.11$.

Intention. Regression analyses with intention as the dependent variable revealed that neither self-efficacy, nor frame, nor the frame by self-efficacy interaction term contributed significantly to the prediction of intention, $p s>.11$.

Behaviour. Regression analyses with salt consumption at follow-up as the dependent variable revealed that the interaction between frame and baseline self-efficacy contributed significantly to the prediction of behaviour, $B=-1.49$, $t(512)=-2.29, p<.05$. Next, simple slope analyses were performed to analyse the main effect of frame for participants in the low self-efficacy condition and participants in the high self-efficacy condition. Supporting our hypothesis, loss- 
framed communications resulted in lower salt consumption than gain-framed communications for participants in the high self-efficacy condition, $B=-.94$, $t(512)=-2.03, p<.05$. For participants in the low self-efficacy condition, there was no significant difference between the gain- and loss-framed condition, $B=$ $.56, t(512)=1.21, p=.23$ (see Figure 7$)$.

\section{Discussion}

In the present paper, it was hypothesized that loss-framed messages would result in stronger intentions to reduce salt intake and in actual healthbehaviour change than gain-framed messages but only for participants with high self-efficacy to decrease salt consumption. When self-efficacy is low, threatening health-promoting messages might be processed defensively, the negative information in loss-framed messages may therefore be avoided or dismissed and as a result loss-framed messages might lose their advantage over gain-framed messages. Our results partly supported this hypothesis. The results of the pretest showed that the loss-framed message was perceived as more threatening than the gain-framed message. Furthermore, the results of the main experiment showed that loss-framed information resulted in a lower salt-intake after three weeks, but only for those participants who received the self-efficacy enhancing information. However, the analyses of information acceptance, our measure of defensive reactions to the information, did not yield evidence that the loss-framed information evoked more defensive reactions than the gain-framed information. Since our hypothesis relied on the assumption that loss-framed information results in greater defensive reactions, the lack of evidence in this regard hinders interpretation of our results. The results of the analyses for intention similarly did not yield the hypothesized results. Thus, the effects of framing and self-efficacy on behaviour were not mediated by information acceptance and intention.

Previous studies have also failed to identify mediators of framing effects. For instance, in a study investigating the effects of framing and ethnic targeting on mammography use, Schneider and colleagues (2001a) tested the mediating effect of numerous psychosocial variables, such as risk perceptions, selfefficacy, outcome efficacy, attitudes, social norms, and intentions, but none of these variables was influenced by framing, and thus did not qualify as a potential mediator of the effect on behaviour that was found at a six-month follow-up. Similarly, Banks and colleagues (1995) found effects of framing on behaviour, but failed to find an effect on intention (for similar results see Detweiller, Bedell, Salovey, Pronin, \& Rothman, 1999; Rothman, Martino, Bedell, Detweiller, \& Salovey, 1999). It seems that, on some occasions, framing can have psychological effects on recipients that are too subtle to be assessed with self-report posttest questionnaires, but stable enough to affect behavioural decisions. Future 
studies should investigate how framing exerts influence on behaviour, for instance by testing whether framing is capable of affecting implicit versus explicit attitudes.

Figure 7 Regression slopes arising from the relationship between selfefficacy and salt consumption for participants who received a gain-framed communication versus those who received a loss-framed communication

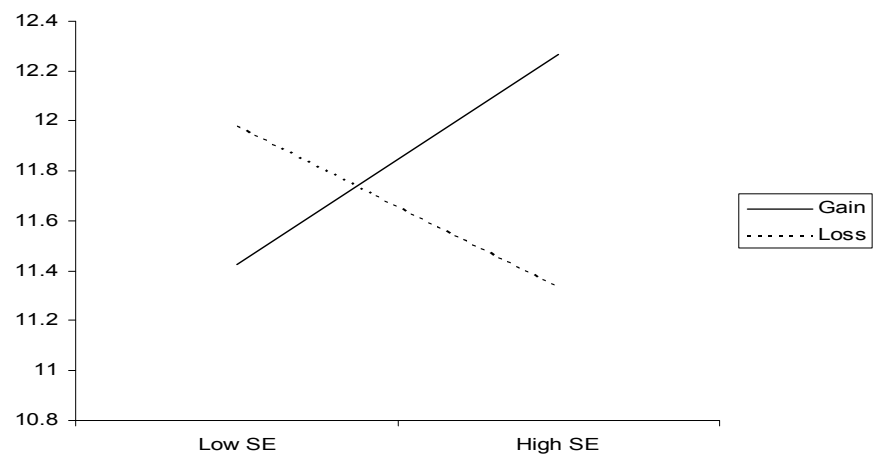

With regards to intention, the present study failed to replicate the findings of two previous studies which found that self-efficacy moderated the effects of gain- and loss-framed communications on participants' health intentions (Van 't Riet et al., 2008; in press). However, we found evidence for an effect of selfefficacy and framing on behaviour, which from a health-promotion perspective is a far more important outcome. In addition, an important strength of the present study is the fact that we used a self-efficacy manipulation instead of a self-report assessment of self-efficacy, offering ground for stronger conclusions on the causality of the effect. Thus, the results of the present study, together with the results of two previous studies (Van 't Riet et al., 2008; in press), strongly suggest that self-efficacy can influence the differential effects of gain- and loss-framed health-promoting messages on persuasion.

The present study was subject to certain limitations. First, we operationalized defensive reactions as measures of participants' information acceptance. There are various other measures that can be used to assess defensive reactions, however, such as avoidance and fatalism (see for instance Rippetoe \& Rogers, 1987) or more direct assessments, like the number of counter-arguments generated by recipients (Keller \& Block, 1999) or reading time (Reed \& Aspinwall, 1998). Although the results of a recent meta-analysis indicate that information acceptance is a reliable measure of defensive reactions (Good \& Abraham, 2007) 
by using information acceptance scores only we may have overlooked other important defensive processes. Second, we constructed an index to assess salt consumption, but we did not gather data to investigate the validity and reliability of this measure. While objective measures, using for instance biochemical (e.g., Sanchez Castillo, Seidell, \& James, 1987) or behavioural (e.g., Miller et al., 1998) procedures, are generally more suitable for assessing total salt intake, selfreport may also be used to assess salt use in cooking and at the table (Sproston \& Primatesta, 2004; cf. Smerecnik, Mesters, De Vries, \& De Vries, in preparation). Still, further research is needed to increase validity and reliability of current saltconsumption measurements (cf. Parkington \& Roussos, 2008).

Despite these limitations, our findings underline the importance of selfefficacy in persuasion. They suggest that self-efficacy, besides being a major determinant of motivation to engage in healthy behaviour (De Vries \& Backbier, 1994; Schwarzer, 1992; Strecher, DeVellis, Becker, \& Rosenstock, 1986), can also influence the differential persuasive effects of gain- and loss-framed healthpromoting messages. In addition, the results of this study suggest that health education interventions could use a combination of self-efficacy information and loss-framed information to increase the effectiveness of health-education interventions. 



\title{
Chapter 8: Investigating the Effects of Message Framing in High- and Low-Threat Informa- tion $^{14}$
}

\begin{abstract}
Health-promoting messages can be framed in terms of the gains that are associated with healthy behaviour (gain-framed) or the losses that are associated with unhealthy behaviour (loss-framed). In the present research we examined whether the effect of message frame is different for low- versus high-threat information, hypothesizing that a gain frame might be more persuasive for lowthreat information whereas a loss frame might be more persuasive for high-threat information. Study $8.1(\mathrm{~N}=98)$ used framed information advocating physical activity and found that gain-framed information resulted in higher ratings of information acceptance, particularly when the information was of a low-threat nature. Study $8.2(\mathrm{~N}=99)$ used framed messages advocating a reduction in alcohol intake and found that gain-framed information resulted in more positive attitudes, but only when the information was of a low-threat nature. No advantage of loss frames was found in the high-threat condition. Results suggest that the effects of gain- and loss-framed messages are dependent on the nature of the information that is conveyed in the message.
\end{abstract}

\section{Introduction}

Health promoting messages usually stress the value of healthy behaviour, but they can do this in several ways. In particular, the message can be framed in terms of the benefits of engaging in the behaviour (gain frame) or in terms of the costs of failing to engage in the behaviour (loss frame). Research has shown that these frames can have a different persuasive impact, even when the different frames are applied to objectively equivalent information (see for instance Broemer, 2004; Cox, Cox, \& Zimet, 2006; Rothman, Bartels, Wlaschin, \& Salovey, 2006; Rothman \& Salovey, 1997; Van 't Riet, Ruiter, Werrij, \& De Vries, 2008). Unfortunately, studies show inconsistent results with regard to which type of framing is more persuasive. A recent meta-analysis showed that for behaviours serving to detect an illness gain- and loss-framed messages were not differentially persuasive (O'Keefe \& Jensen, 2006). For behaviours serving to prevent an illness a small advantage of gain-framed information was found, although this effect seemed mainly due to a limited amount of studies on dental health (O'Keefe \& Jensen, 2007). Thus, despite an impressive body of literature, 
it is currently unclear how health-care professionals should frame persuasive messages.

In the present research we tried to shed some light on the inconsistent findings in the message-framing literature by investigating under which circumstances gain- or loss-framed information is more persuasive. In particular, since little is known about whether message-framing effects vary as a function of message content (Rothman et al., 2006), we tried to expand our understanding of framing effects by focusing on the role of conveyed threat. In two experiments, we tested whether the persuasive effects of gain- and loss frames differ between high- and low-threat health-promoting information.

Numerous scholars have investigated moderators of message-framing effects. Rothman and Salovey (1997; Rothman et al., 2006), for instance, have proposed that the effect of framing is contingent on the function of the behaviour that is promoted, differentiating between illness-prevention behaviour (e.g., exercising) and illness-detection behaviour (e.g., performing skin selfexamination). Drawing from Prospect Theory (Kahneman \& Tversky, 1979; Tversky \& Kahneman, 1981) they argue that gain-framed information triggers people to be risk-averse while loss-framed information triggers people to be riskseeking. Since people perceive prevention behaviours as inherently risk-averse (because prevention behaviours serve to reduce the risk of falling ill), gainframed information should be more persuasive when advocating prevention behaviour. Since detection behaviours are perceived as inherently risky (because detection behaviour may entail the risk of having to cope with bad news) lossframed information should be more persuasive than gain-framed information in advocating detection behaviour. As mentioned above, however, recent metaanalytic findings (O'Keefe \& Jensen, 2006; 2007) are not in line with this hypothesis. In response, Latimer and colleagues (Latimer, Salovey, \& Rothman, 2007) have argued that the way people perceive a health behaviour (i.e., as a relatively safe or relatively risky behaviour) is of particular importance: when people perceive a behaviour as safe, irrespective of whether it prevents or detects illness, gain-framed information should be more persuasive, whereas when people perceive a behaviour as risky, loss-framed information should be more persuasive (see also Apanovitch, McCarthy, \& Salovey, 2003).

Another approach to the study of message framing has been the focus on individual differences in recipients' approach and avoidance orientation (Gerend \& Shepherd, 2007; Mann, Sherman, \& Updegraff, 2004; Sherman, Mann, \& Updegraff, 2006). In a series of recent studies, Mann and colleagues (2004; Sherman et al., 2006; Updegraff, Sherman, Luyster, \& Mann, 2007) used a questionnaire developed by Carver and White (1994) to measure the strength of participants' approach and avoidance orientation. They then provided participants with framed information advocating dental hygiene behaviours. The results of their studies showed that for recipients with a predominant approach orientation, 
gain-framed information was more persuasive than loss-framed information whereas the reverse was true for recipients with a predominant avoidance orientation. They argued that persuasion is most likely to occur when the frame of the health-promoting information is congruent with the recipients motivational orientation.

Message-framing research has investigated the moderating influence of various variables. The function of the promoted behaviour, perceptions of the behaviour as safe versus risky, and individual differences in approach and avoidance orientation are three particularly noteworthy examples. Other examples are mode of processing (Maheswaran \& Meyers Levy, 1990; Meyers Levy \& Maheswaran, 2004), ambivalence (Broemer, 2002), ease of imagination (Broemer, 2004), and self-efficacy (Van 't Riet, Ruiter, Werrij, \& De Vries, 2008). It is also conceivable, however, that message framing effects are dependent on the content of the framed message. Although this possibility has received little attention until now (Rothman et al., 2006), finding that message content interacts with message framing can have important implications for practice. For instance, if research would show that low-threat information is particularly persuasive when it is gain-framed as opposed to loss-framed, these findings could result in clear recommendations for health-care professionals designing low-threat messages.

In one study that did focus on message content, Gerend and Cullen (2008) investigated the effects of gain- and loss-framed messages about alcohol use, stressing either the long-term consequences or the short-term consequences of (not) drinking. They found that gain-framed messages were more persuasive, but only when stressing the short-term consequences of not drinking. The present research tries to expand this line of research, also focusing on the influence of message content. We investigated the effects of framing for information that is of a high- versus a low-threat nature. Thus, in the present study we combined message-framing research and fear-appeal research.

\section{Fear appeals and framing}

Numerous studies tested the effects of fear appeals on measures of attitudes, intention and behaviours (for overviews see Eagly \& Chaiken, 1993; Ruiter, Abraham, \& Kok, 2001). A fear appeal is a persuasive message attempting to arouse fear to promote precautionary motivation and self-protective action (Rogers, 1983). Under certain circumstances, such as a high perceived efficacy (Ruiter, Abraham, \& Kok, 2001) evoking fear can enhance a message's persuasive power (Mongeau, 1998; Witte \& Allen, 2000). So far, only one study to our knowledge investigated the combined effects of fear appeals and message framing (Ruiter, Kok, Verplanken, \& Van Eersel, 2003). The results of this study showed that for participants who had received high-threat information concerning the consequences of breast cancer loss-framed information promoting breast self-examination was more persuasive than gain-framed information. For partici- 


\section{Chapter 8}

pants who had received low-threat information no difference between gain- and loss-framed information was found. Ruiter and colleagues, however, manipulated threat by having participants read high- or low-threat messages before they read the framed information. In the present research, we combined the threatening information and the framed information into one message, manipulating threat and frame at the same time. Following the finding that congruence between the recipient's motivational orientation and message frame can enhance persuasion (e.g., Mann et al., 2004), we investigated whether congruence between message content and message frame can also enhance persuasion. In line with the results by Ruiter and colleagues (2003) we hypothesized that persuasion is most likely if the message frame is congruent with the message content. We thus hypothesized that a gain-frame is more persuasive for low-threat information, whereas a loss frame is more persuasive for high-threat information.

\section{The Present Research}

We conducted two experiments in which participants read information that had either low- or high-threat content and that was either gain- or lossframed. We hypothesized that persuasion is most likely if the message frame is congruent with the message content and that a gain frame is more persuasive for low-threat information whereas a loss frame is more persuasive for high-threat information. This research was approved by the Medical Ethical Committee of Maastricht University.

\section{Study 8.1}

\section{Method}

Procedure and design. Participants were recruited in the restaurant buildings of Maastricht University. Any student sitting at a restaurant table during lunchtime was eligible. Ninety-eight students agreed to take part and filled in a questionnaire. These students received a booklet (A4-format) containing an introduction to the study and a six-page questionnaire. Each of the six pages started with a statement about physical activity, followed by three questions about participants' acceptance of the statement. All participants received the same six statements, but three stressed relatively non-threatening consequences of (not engaging in) physical activity, such as (not) feeling energized, whereas the other three stressed relatively threatening consequences such as reducing (increasing) the risk of cardiovascular disease. Thus, threatening content constituted a within-subjects manipulation and all participants reacted to both the highand low-threat content. To avoid sequence effects, the order of the statements was varied randomly across participants. Furthermore, participants were randomly assigned to one of two conditions. In the gain-frame condition, the state- 
ments participants received were gain-framed; conversely, in the loss-frame condition the statements were loss-framed. One example of a low-threat gain-framed [loss-framed] statement on physical activity was 'If I am [in]sufficiently active, I will feel more [less] energized.' One of the high-threat gain-framed [lossframed] statements read 'If I am [in]sufficiently active, I will have a smaller chance [greater risk] of cardiovascular disease.' Pilot studies showed that these statements were judged as credible.The experiment used a 2 (within-participants: low-threat content vs. high-threat content) x 2 (between-participants: gain- vs. loss-frame) design with information acceptance as the outcome variable of interest.

Information Acceptance. Three items following each statement assessed information acceptance using a semantic differential with a 10-point scale. Specifically, they assessed the extent to which participants agreed with the statement $(1=$ totally agree $; 10=$ totally disagree $)$ and rated the statements as exaggerated $(1=$ very exaggerated $; 10=$ not at all exaggerated $)$ or relevant $(1=$ very relevant; $10=$ not at all relevant). The scores on the agreement and relevance items were reversed to create an average information acceptance score. To investigate the internal consistency of this scale, Cronbach's alpha was calculated for each statement separately and ranged from $\alpha=.66$ to $\alpha=.84$. The average internal consistency was $\alpha=.73$.

Statistical analysis. Linear multilevel analyses were used to account for the fact that items were clustered within individuals. In Step 1 of the analyses, the effects of frame (coded as $0=$ gain frame, $1=$ loss frame) and threat $(0=$ low-threat content, 1 = high-threat content) were investigated. In Step 2, the frame by threat interaction term was entered into the analysis. In the event of a significant interaction effect, the effect of frame was investigated for the nonthreatening and threatening statements separately by means of simple effects analyses (Aiken \& West, 1991). We used the Mixed Linear Models module within the software package SPSS 13.0.

\section{Results and Discussion}

In Step 1 of the analysis, frame contributed significantly to the prediction of information acceptance, such that gain-framed statements resulted in higher ratings of information acceptance than loss-framed statements, $B=-1.18$, $t=-5.39, p<.001$. Also, statements with high-threat content evoked significantly higher levels of information acceptance than statements with low-threat content, $B=1.29, t=7.99, p<.001$. In Step 2 , there was a significant frame by threat interaction effect, $B=.74, t=2.36, p<.05$. In the low-threat content condition, gain-framed statements resulted in higher levels of information acceptance than loss-framed statements, $B=-1.68, t=-5.51, p<.001$. In the high-threat content condition, gain-framed statements also resulted in significantly higher levels of information acceptance, although this effect was smaller, $B=-.93, t=-3.84, p<$ 
.001 . Thus in both the low- and the high-threat condition, gain-framed information was more convincing than loss-framed information, but this effect was particularly pronounced for the low-threat statements (see Figure 8.1).

Figure 8.1 Regression slopes arising from the relationship between threat and information acceptance for participants who received gain- versus lossframed information in Study 8.1

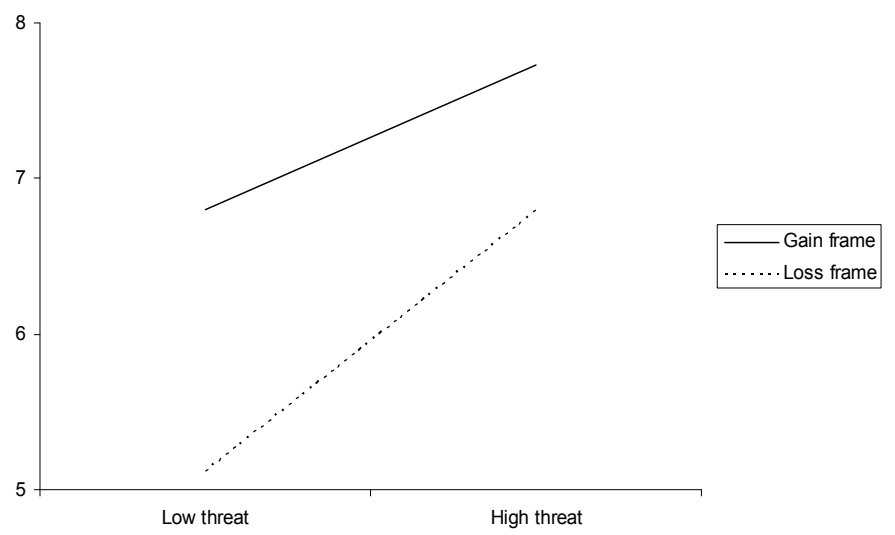

These results show that threat can moderate the effect of frame on information acceptance. These findings need replication, however, before we can draw any strong conclusions. For one, the strong main effect of threat makes the interaction between frame and threat difficult to interpret. Close inspection of Figure 8.1 leaves room for the possibility that this interaction was the result of a ceiling effect. Moreover, Study 8.1 was subject to several limitations. First, the fact that we used a within-participants manipulation of threat makes it difficult to compare our results to other message framing studies, the majority of which have used between-participants manipulations. Second, the research setting of Study 8.1 (the university restaurant during lunchtime) limited experimental control, and although we instructed participants to fill out the questionnaires individually, we can not be sure that all participants refrained from communicating with each other during the procedure. Third, we assessed participants' acceptance of the information. Although perceived effectiveness, a measure akin to information acceptance, has been shown to be substantially correlated with actual effectiveness (Dillard, Weber, \& Vail, 2007), finding effects of framing on information acceptance is less relevant than, for instance, finding effects on attitude or intention. To be able to draw stronger conclusions, therefore, we aimed to replicate these effects in Study 8.2, making several changes as compared with Study 8.1. First, we used written framed messages consisting of 379 and 389 words instead 
of single framed statements. Second, to increase experimental control, we conducted Study 8.2 in our research lab. Third, we assessed participants' attitude towards and intention to engage in the recommended behaviour. Given the high prevalence of binge drinking in student populations (Goldman, Boyd, \& Faden, 2002) we used framed messages about alcohol use as the framed materials.

\section{Study 8.2}

\section{Method}

Participants. University students listed in a database of voluntary research participants were invited by email to take part in the experiment. In total, 114 students participated in the experiment in exchange for $€ 5$. Fifteen participants indicated that they never drank alcohol and were excluded from the analyses. Among the remaining 99 participants, most were female (83 vs 16 males) and age ranged from 18 to 28 years, with a mean age of 21.0 years $(S D=1.9)$.

Design and procedure. The present study used a 2 (threat: high-threat content vs low-threat content) X 2 (frame: gain frame vs loss frame) betweenparticipants design. Participants were scheduled to visit the laboratory, and were seated in individual booths. We told participants that they were about to participate in a study to test health education materials that we had designed for use 'on the Internet'. Next, we provided participants with a persuasive communication about alcohol, which had either high-threat or low-threat content and was either gain- or loss-framed. After reading it, participants completed the dependent measures, then were debriefed and received their fee.

Persuasive communication. For the present study, we designed short communications of 379 and 389 words, consisting of either high-threat or lowthreat content and stressing either the benefits of not drinking too much (gain frame), or the costs of drinking too much (loss frame). The full texts are available in Appendix G.

Outcome measures. To assess the effects of the threat and frame manipulations, we conducted two manipulation checks. To assess whether the manipulation of threat had been successful, one item asked participants to indicate the extent to which they found the message threatening $(1=$ very threatening; 7 $=$ not threatening at all). To assess whether the manipulation of frame had been successful, one item asked participants to indicate the extent to which they found that the message had a positive tone $(1=$ very positive; $7=$ very negative $)$.

Six items were used to assess attitude towards reducing alcohol intake, asking participants to indicate the extent to which they rated drinking less alcohol as (1) very good vs. (7) very bad, (1) very healthy vs. (7) very unhealthy, (1) very nice vs. (7) not at all nice, (1) very much worth the effort vs. (7) not worth the effort at all, (1) very important vs. (7) very unimportant, (1) very sensible vs. 
(7) not sensible at all. After scores on the attitude items were reversed, an average score was created $(\alpha=.81)$.

Six items were used to assess intention to reduce alcohol intake. One item asked participants 'how likely is it that you will drink less alcohol in the future?' ( 1 = Very likely; 7 = Very unlikely $)$. One item asked participants: 'how likely is it that you will drink less alcohol in the coming week?' ( 1 = Very likely; $7=$ Very unlikely). Two items asked participants whether they planned to drink less alcohol in the future and in the coming week. One item asked participants whether they considered drinking less alcohol in the future and one item asked participants whether they were sure that the would drink less alcohol in the coming week. These four items used a 7-point scale $(1=$ certainly; $7=$ certainly not $)$. Scores on the intention items were reversed such that a high score indicated a strong intention to drink less alcohol and an average intention score was calculated $(\alpha=.91)$.

Statistical analysis. Linear regression analyses tested the main effects of threat (coded as $0=$ low threat; $1=$ high threat) and frame (coded as $0=$ gain frame, $1=$ loss frame), and the threat by frame interaction term on the manipulation checks, attitude, and intention. In case of a significant interaction effect, simple slope analyses were conducted to investigate the nature of the interaction (Aiken \& West, 1991).

\section{Results}

Manipulation checks. Results of a linear regression analysis showed that the high-threat information was perceived as significantly more threatening than the low-threat information, $B=-.60, t(111)=-2.53, p<.05$. Frame did not contribute significantly to the prediction of perceived threat, $B=$ $.37, t(111)=-1.58, p=.12$, nor did the threat by frame interaction, $B=.10$, $t(110)=.22, p=.83$. Next, we set out to investigate whether the manipulation of frame had been successful. Results showed that the gain-framed communication was perceived as more positive than the loss-framed communication, $B=1.33$, $t(111)=5.10, p<.001$. In addition, the low-threat communication was perceived as significantly more positive than the high-threat communication, $B=.86$, $t(111)=3.27, p<.01$. There was no significant interaction effect, $B=-.33$, $t(110)=-.63, p=.53$.

Attitude. In Step 1 of the analyses, neither frame nor threat had a significant effect on attitude, $p s>.22$. In Step 2, regression analyses revealed that the interaction between threat and frame contributed significantly to the prediction of attitude, $B=.62, t(95)=2.10, p<.05$. Simple slope analyses indicated that in the low-threat condition gain-framed information resulted in a more positive attitude than loss-framed information, $B=-.44, t(95)=-2.31, p<.05$. In the high-threat condition, there was no significant effect of frame, $B=.18, t(95)=$ $.79, p=.43$ (see Figure 8.2). 
Intention. Our analyses revealed that there were no main effects of threat or frame on intention, $p \mathrm{~s}>.25$. Also, in contrast to the results for attitude, the interaction between threat and frame did not have a significant contribution to the prediction of intention, $B=.47, t(95)=.79, p=.43$.

Figure 8.2 Regression slopes arising from the relationship between threat and information acceptance for participants who received a gain- versus those who received a loss-framed communication in Study 8.2

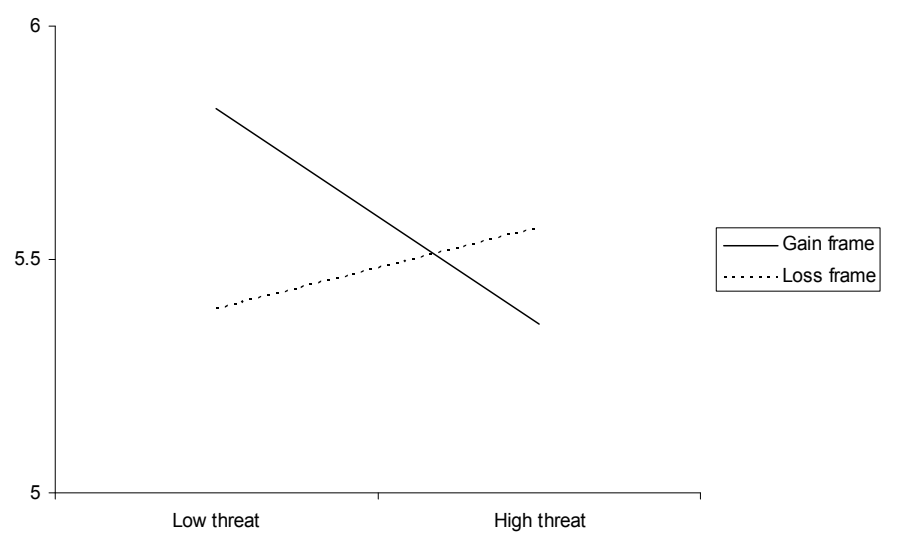

\section{General Discussion}

In the present research we investigated the effects of framing in highversus low-threat information. Following previous studies that found that healthpromoting information is more persuasive when the information frame is congruent with the recipients' dispositional approach or avoidance orientation, we investigated whether congruence between the information frame and the content of the information could also enhance persuasion. In line with the results of a previous study (Ruiter et al., 2003) we hypothesized that gain-framed information would be more persuasive for low-threat information whereas loss-framed information would be more persuasive for high-threat information. Across two experiments, our results showed partial support for our hypothesis. In Study 8.1, gain-framed information resulted in higher levels of information acceptance than loss-framed information, particularly when the information was of a low-threat nature. In Study 8.2, gain-framed information resulted in a more positive attitude, now only in the low-threat condition.

Contrary to our hypothesis, we found no evidence that loss-framed information was more persuasive when the information had high-threat content. 


\section{Chapter 8}

Possibly, the information we used was unsuccessful in sufficiently increasing perceptions of threat. One limitation of the present research is the fact that the results of Study 8.1 do not provide us with information about the level of threat that was actually perceived by participants. We assumed that information about relatively major health consequences such as increasing the risk of cardiovascular disease would be perceived as more threatening than information about relatively mild health consequences such as decreased stamina. However, we could not directly infer from our data whether this was the case. In fact, given that many students might not perceive themselves at risk for major health threats such as cardiovascular disease, a possible lack of perceived threat might be the reason that we did not find an advantage of a loss frame in the high-threat condition. In Study 8.2, the results for the manipulation checks showed that the highthreat messages were perceived as more threatening than the low-threat messages, and additional analyses revealed that, for participants in the high-threat condition the mean score on this manipulation check $(\mathrm{M}=4.44)$ was higher (i.e., more perceived threat) than the neutral scale mid-point, $t(43)=2.60, p=.01$, whereas for participants in the low-threat condition, the mean $(\mathrm{M}=3.86)$ was not significantly different from the mid-point, $t(56)=.85, p=.40$. However, it might well be that this increased perception of threat in the high-threat condition was still not sufficient for an advantage of loss-framed information to arise. In fearappeal research, threat is often conceptualized as consisting of severity and susceptibility (e.g., Witte, 1992; 1994). In the present research, we manipulated threat by varying the severity of the consequences of unhealthy behaviour (e.g., cardiovascular disease vs. loss of energy). However, participants' perceptions of susceptibility might have been generally low (especially in Study 8.1) and therefore no advantage of loss-framed information was found in the high-threat condition. In contrast, Ruiter and colleagues manipulated both severity and susceptibility in their study, which might have resulted in higher perceptions of threat. This might explain why, in contrast to the present research, those authors did find an advantage of loss-framed information in the high-threat condition. Moreover, previous research shows that for people with high risk perceptions (i.e., high perceptions of susceptibility), loss-framed information is more persuasive than gain-framed information (Gerend \& Shepherd, 2007). Future research should disentangle the role of perceived severity and susceptibility in messageframing effects.

It is unclear why, in Study 8.2, we found a threat by frame interaction effect on attitude, but not on intention. One possible interpretation of these findings is that attitude and intention might sometimes be quite differently affected by persuasive information. This is in line with results from a recent study (Van 't Riet, Ruiter, Werrij, Candel, \& De Vries, submitted), which found that gainframed information evoked greater levels of positive feelings than loss-framed information, and that these positive feelings resulted in more positive attitudes. 
Loss-framed messages, on the other hand, evoked more negative feelings than gain-framed messages and negative feelings, while not being associated with attitudes, had a direct and positive effect on intentions. It seems thus that framed information can have different effects on attitude and intention (for a similar discussion of different persuasive effects on attitudes and intentions, see De Hoog, Stroebe, De Wit, 2005). It is also important to note that in the present study attitude and intention were very weakly and non-significantly correlated, $r$ $=.18, p=.07$. Future research might be able to investigate in more detail which persuasive information is likely to affect attitudes, and which is most likely to affect intentions.

One limitation of the present research was the fact that we used information acceptance as the outcome measure in Experiments 1. It could be argued that information acceptance is not the most relevant outcome of the persuasion process. However, according to Ajzen's (2002a) notion of the 'error choice method' it is improbable that participants will have an objective basis for estimating how relevant or convincing persuasive information might be for most people. Therefore, their judgements are likely to reflect their own attitudes. Empirical support for this comes from a recent meta-analysis that showed that perceived effectiveness (i.e., information acceptance) was substantially correlated with actual effectiveness (i.e., attitude; Dillard, Weber, \& Vail, 2007). Thus even though the primary aim of health education is to change behaviour, and accepting information is not the same as actual behaviour change, we argue that information acceptance can not be discarded as an irrelevant outcome. In fact, the effects of frame on information acceptance might have direct implications for health-promoting materials on the Internet. Given the growing practice to use the Internet as a medium for health-promoting information and the importance of increasing people's exposure to this information (i.e., drawing attention and keeping recipients interested; see for instance Crutzen et al., 2008) information acceptance might be of critical importance. After all, people seeking information on the Internet are most likely to select information that seems relevant, interesting, and trustworthy. Future research should investigate whether message framing can be used to increase exposure in an online setting.

Study 8.2 also has valuable implications for health education practice. In line with a previous study (Gerend \& Cullen, 2008), the results of Study 8.2 suggest that message promoting low alcohol consumption are more persuasive when they are gain- rather than loss-framed. However, whereas Gerend and Cullen found that gain-framed information was only more persuasive when the information stressed the short term positive consequences of reducing alcohol consumption our results suggest that the advantage of gain-framed information is limited to low-threat messages.

In sum, the results of the present research are in line with a previous study (Gerend \& Cullen, 2008) in suggesting that the content of framed message 
108 Chapter 8

can influence the effects of message framing. Across two experiments gainframed information was found to be more persuasive than loss-framed information, particularly for low-threat content. To more fully expand our understanding of framing effects, future studies could highlight the role of other content-related variables beyond the level of message threat. 
General Discussion 

For a long time researchers have been aware that people respond differently to messages containing the same information only worded in a different format. As early as 1941, Rugg (1941) reported the results of a classic study in which one national sample was asked if they were willing to allow public speeches against democracy whereas another comparable sample was asked if they were willing to forbid such speeches. Rugg's results showed that more respondents were not willing to allow public speeches against democracy than were willing to forbid them. The present thesis shows that the framing of health-education messages can also influence people's health cognition and motivation. In Study 3.1, 3.2, 6 and 8.1, for instance, gain-framed information was rated as more acceptable than factually equivalent loss-framed information. But what do these findings imply for research and practice? In this General Discussion, we will first discuss the main findings from this thesis. Next, we will discuss limitations of the research and remaining questions. We will finish by discussing implications for research and practice.

\section{Main findings}

A recent meta-analysis found 165 studies dealing with message framing (O'Keefe \& Jensen, 2006), a number which is likely to have further increased over the past two years. Although many of the processes that are studied in this thesis have also been addressed elsewhere, we propose in all modesty that the present thesis contributes to the literature by highlighting the influence of three hitherto understudied phenomena: a) the affective consequences of gain- and loss-framed messages, b) the moderating role of self-efficacy, and c) the moderating role of message threat. The fourth contribution to the literature is d) the obtained evidence that gain-framed information can result in higher ratings of information acceptance than loss-framed information. Below, we will discuss these findings. Because the ultimate goal of health education is to produce effects on health behaviour, we will also discuss behavioural effects of framing. Finally, we will briefly discuss analyses that investigated the moderating influence of several psychological variables on the effects of message framing but found no effects.

\section{Underlying Mechanisms: Affective consequences of framed messages}

The results of previous research suggest that gain-framed information can give rise to positive affect whereas loss-framed information can give rise to negative affect (Chang, 2005; Cox, Cox, \& Zimet, 2006; Cox \& Cox, 2001; Millar \& Millar, 2000; Schneider, Salovey, Apanovitch et al., 2001; Shen \& Dillard, 2007). Many other studies, however, have discarded this phenomenon. In the first well-known message-framing study, for instance, Meyerowitz and Chaiken 


\section{Framing Health Communication Messages}

(1987) reported that they found no effects on perceived threat, using a four-item threat/fear scale. Although other researchers have also reported null-findings (Detweiller, Bedell, Salovey, Pronin, \& Rothman, 1999; Gerend \& Shepherd, 2007), it is hard to draw conclusions from these results. First, null-findings are often hard to interpret (Sternberg, 1988). Second, self-report measures of affect have several potential problems (Robinson \& Clore, 2002). Therefore, we aimed to further investigate the role of positive and negative reactions to framed information.

In Study 3.2 and 4 we found evidence that gain-framed messages result in stronger positive affective reactions than loss-framed messages whereas lossframed messages result in stronger negative affective reactions. Positive affective reactions in turn positively influenced measures of information acceptance and attitude, whereas negative affective reactions positively influenced intention. Our results show that, since framing has opposing effects on positive and negative affect, and positive and negative affect both may contribute to persuasion, it can be difficult to find consistent main effects of framing on behaviour. In this regard, our results might be exemplary of the message-framing literature as a whole (O'Keefe \& Jensen, 2006; 2007). Our findings add to the literature, however, by implying that message-framing research should pay specific attention the underlying mechanisms of framing effects and to the outcome measures being used.

\section{The moderating role of self-efficacy}

To explore the reasons for the inconsistent findings in the message framing literature research is needed that investigates under which circumstances gain- or loss-framed information is more persuasive. Research investigating possible moderating variables that can explain differences in the effects of gain- and loss-framed messages is indispensable to deepening our understanding of message-framing effects on persuasion. The second aim of our research, therefore, was to investigate whether we could identify potential moderators of the persuasive effects of framed health-promoting messages. In three studies we examined the influence of self-efficacy to engage in healthy behaviour on the effects of gain- and loss-framed health-promoting messages. Study 5, among adult smokers, found that participants with a high self-efficacy to quit smoking reported a stronger intention to quit smoking after receiving a loss-framed message than after receiving a gain-framed message or no message. For participants with a low self-efficacy to quit smoking there were no differences in motivation to quit smoking between the gain-, loss-, and no frame control condition. The results of Study 6 and 7 yielded similar results, suggesting that messages stressing losses may be more persuasive than messages stressing gains, but only in persons with high self-efficacy. 
These findings underline the importance of self-efficacy in persuasion. They suggest that self-efficacy can influence the differential persuasive effects of gain- and loss-framed health-promoting messages, but they also serve as a powerful reminder that self-efficacy is one of the most important factors in the decision to engage in healthy behaviour (De Vries \& Backbier, 1994; Schwarzer, 1992; Strecher et al., 1986).

\section{The moderating role of message threat}

In Study 8.1 and 8.2 we examined whether the effect of message frame is different for low- versus high-threat information, hypothesizing that a gainframe might be more persuasive for low-threat information whereas a loss frame might be more persuasive for high-threat information. Study 8.1 used framed information advocating physical activity and found that gain-framed information resulted in higher ratings of information acceptance, particularly when the information was of a low-threat nature. Study 8.2 used framed messages advocating a reduction in alcohol intake and found that gain-framed information resulted in more positive attitudes, but only when the information was of a low-threat nature. No advantage of loss frames was found in the high-threat condition. Results suggest that the effects of gain- and loss-framed messages are dependent on the nature of the information that is conveyed in the message. These results have valuable implications for health education practice. In line with a previous study on framing messages about alcohol consumption (Gerend \& Cullen, 2008), the results of the studies in Chapter 8 suggest that information about physical activity and alcohol use are more persuasive when they are gain- rather than lossframed. Whereas Gerend and Cullen found that gain-framed information was only more persuasive when the information stressed the short term positive consequences of reducing alcohol consumption, our results suggest that the advantage of gain-framed information is also limited to low-threat messages.

\section{Information acceptance}

The results of Study 3.1, 3.2, 6 and 8.1 suggest that gain-framed information is judged as more credible, more relevant, and more interesting than lossframed information. These results are also in line with findings from other studies (Latimer et al., 2008b; Reinhart, Marshall, Feeley, \& Tutzauer, 2007; Schneider et al., 2001b; Smith, 1996). In addition, two studies by Jones and colleagues showed that source credibility was rated as higher when information was gainversus loss-framed (Jones, Sinclair, \& Courneya, 2003; Jones, Sinclair, Rhodes, $\&$ Courneya, 2004). Other studies show mixed findings, suggesting that this effect may be limited to messages stressing health outcomes (as opposed to social outcomes; Kiene, Barta, Zelenski, \& Cothran, 2005), or that it only occurs in participants who are highly involved in the issue (Millar \& Millar, 2000). Still other studies failed to find effects of framing on information acceptance, but 


\section{Framing Health Communication Messages}

these studies largely used flawed measures of information acceptance such as single item measures (e.g., Gerend \& Cullen, 2008; Gerend \& Shepherd, 2007; Homer \& Yoon, 1992; Rivers, Salovey, Pizarro, Pizarro, \& Schneider, 2005; Rothman, Martino, Bedell, Detweiller, \& Salovey, 1999), a point which we will return to later.

Although future research is needed to corroborate our findings, it is important to realise that these effects on information acceptance can be very relevant. Theoretical support for this comes from Ajzen's (2002a) notion of the 'error choice method', according to which, because it is improbable that participants will have an objective basis for estimating how relevant or convincing these messages might be for most people, their judgements are likely to reflect their own attitudes. Empirical support for this comes from a recent meta-analysis that showed that information acceptance was substantially correlated with actual effectiveness. The importance of information acceptance is illustrated by recent theorizing about the effectiveness of computer-tailored communications. Several researchers have proposed that the well-established effectiveness of computertailoring (Noar, Benac, \& Harris, 2007) is due to the fact that tailored information is rated as better, saved more often and evaluated as more interesting than non-tailored messages (Dijkstra \& De Vries, 1999; Kreuter, Farrell, Olevitch, \& Brennan, 2000).

Thus, information acceptance can be construed as a highly relevant variable in information processing. Traditionally, however, it has not been considered as a particularly important outcome measure in persuasion research. Likewise, it has been mostly overlooked in the message-framing literature. In fact, effects of message framing on information acceptance have sometimes been considered as confounds which hinder the interpretation of 'proper' framing effects. Rothman and colleagues (1999), for instance, assessed information acceptance in two experiments but did so to establish that only frame differed between the gain- and loss-framed condition, and that there were no additional, confounding, differences in the way recipients perceived the messages. In our view, message-framing researchers should pay particular attention to information acceptance as a potentially important outcome, instead of regarding effects on information acceptance as confounds which are to be avoided. Moreover, it is important to use reliable and valid scales for the assessment of information acceptance. The results of several studies finding no effect of framing on information acceptance need to be interpreted with care, since they mostly used single itemmeasures to assess information acceptance (e.g., Gerend \& Cullen, 2008; Gerend \& Shepherd, 2007; Rivers, Salovey, Pizarro, Pizarro, \& Schneider, 2005; Schneider, Salovey, Apanovitch et al., 2001). Thus, to foster our understanding of message-framing effects, researchers should pay more attention to small steps in the persuasion process, also when this means investigating effects on variables 
that are more distal determinants of behaviour change than attitudes or intentions, such as information acceptance.

\section{Behaviour}

The ultimate goal of health education is to produce effects on health behaviour. Effects of framing on information acceptance, attitude or intention may thus be less helpful than behavioural effects. We did not assess behavioural effects of framing in four of the seven empirical chapters of this thesis. One could say that this constitutes a shortcoming of this research. It should be noted, however, that most contemporary theories of health behaviour propose that attitudes and intentions are necessary antecedents of behaviour (for an overview see Conner \& Norman, 2005). Moreover, because message framing relies on conveying information, it might be more likely to affect health-related cognition and motivation than behaviour directly. Several social cognitive models of health behaviour make a distinction between a pre-behavioural - or motivational phase of health behaviour change and a behavioural or volitional phase of health behaviour change (De Vries, Mesters, Van 't Riet, Willems, \& Reubsaet, 2006; Schwarzer \& Luszczynska, 2008). The null-findings on behaviour in Studies 2 and 3.2 suggest that message framing might be more likely to affect decision making in the motivational than in the volitional phase. On the other hand, Study 7 showed that framing can affect behaviour without affecting health-related cognitions. So far, it thus remains unclear whether message framing can have reliable and predictable effects on behaviour. For health-education practice, this means that message framing can be a useful tool (for instance to increase information acceptance), but is hardly a magic bullet.

\section{Null-findings}

In addition to the studies presented in Chapter 2-8, we investigated whether framing effects could be moderated by several potentially important factors that we have not discussed until now. Our analyses showed, however, that nicotine dependency (Fagerstrom, 1978), stage of change (Prochaska \& DiClemente, 1983), need for cognition (Cacioppo et al., 1996) and regulatory focus (Higgins, 1997) did not significantly interact with message framing. Although it is difficult to interpret these null-findings (cf. Sternberg, 1988), it is worth mentioning that we twice failed to replicate the interaction between need-forcognition and framing (as reported in Steward et al., 2003) and three times failed to replicate the interaction between regulatory focus and framing (as for instance reported in Cesario et al., 2004). 


\section{Limitations}

\section{Affective reactions.}

In Study 3.1, 3.2, and 4, we measured participants' affective reactions to the framed information and investigated whether affective reactions could influence information acceptance, attitude, intention and behaviour. However, a number of factors could hinder the interpretation of our results. First, self-reports of affect can be problematic. Whereas many individuals may be able to describe their affective states accurately, others may provide self-reports that deviate from their actual affective reaction. In this case, they may draw on alternative sources to provide emotional self-reports such as cues derived from the situation or beliefs about how one should feel (Robinson \& Clore, 2002). Recent research suggest that implicit measures of affect, like the Implicit Positive and Negative Affective Test (IPANAT; Quirin, Kazén, \& Kuhl, submitted), could provide a more accurate assessment of affective states.

Another issue that warrants attention is the correlational nature of the associations between affective reactions and persuasive outcome measures. We have proposed that positive affective reactions can increase information acceptance and attitude and that negative affective reactions can influence intention. From the data in Study 3.2 and 4, however, it is impossible to say anything with complete certainty about the causal nature of these relationships. It is conceivable, for instance, that increased levels of information acceptance lead to greater levels of positive affect. Even though it is more difficult to explain why a more positive attitude would lead to greater levels of positive affect and why a stronger intention would lead to stronger negative affect, we argue that future research should investigate these relations more closely to try to establish causality.

One last issue with regards to the role of affect in message framing warrants attention. A limitation of the present research is the fact that we assessed diffuse affect instead of specific emotions. In Study 3.2 and 4, four items assessed general positive affective reactions (i.e., happy, relieved, optimistic, content) and four items assessed general negative affective reactions (i.e., sad, afraid, uncomfortable, tense). Doing this, we used what Van der Pligt and colleagues (1998; page 57) called 'a rather crude dichotomy of positive vs. negative affect.' In fact, these authors argue that instead of using these holistic measures, it is essential to distinguish between various, more specific, affective reactions. A limitation of Experiments 3.1, 3.2, and 4, then, is that we did not differentiate more specifically between possibly relevant emotions, like anger, fear, happiness, and relief.

Many studies in social psychology have studied the effects of positive and negative stimuli on behaviour, and many of those used a broadly construed dichotomy between positive and negative affective stimuli (Dijksterhuis \& Aarts, 
2003; Zajonc, 1980; 1984). On many occasions, however, these studies have focused on the automatic and unconscious processes that are the result of positive and negative affective stimuli. Recent research shows, however, that emotional information processing and the resulting responses unfold from being global to specific: Initial emotional responses are typically based on general, positive-negative evaluations, whereas later emotional responses are based on more specific, fine-grained information processing (Ruys \& Stapel, in press). Since in the present research we focused on the consciously perceived affective reactions to the information, as opposed to automatic and unconscious processes, it is possible that investigating the role of specific emotions instead of general positive and negative affect would have yielded more insight in the effects of framed messages. Dillard and Peck (2000), for instance, investigated whether the content of eight public service announcements (PSA's) elicited anger, fear, sadness, guilt, contentment, surprise and happiness. In addition, they investigated which emotions were predictive of perceived effectiveness. The advantage of this approach is that it makes it possible to investigate more specifically which types of information lead to which emotion. It could then be investigated, for instance, whether gain-framed health promoting messages result in happiness, elation, relief or contentment and whether loss-framed messages result in sadness, disappointment, guilt or fear. Also, since different emotions are associated with different 'action tendencies' (Frijda, 2007), it might lead to more specific hypotheses concerning which emotions affect behaviour and why. One could hypothesize, for instance, that fear can increase intentions to engage in healthy behaviour whereas disappointment could lead to decreased motivation.

In sum, a limitation of the present research is that we did not investigate the role of specific emotions in message-framing effects. Future research should pay attention to this, to be able to gain further insight into the effects of framing on persuasion.

\section{Systematic or heuristic processing}

Another limitation of the present research is the fact that we did not investigate the role of heuristic and systematic processing. As will be outlined below, some of our results might be explained by differences in mode of information processing.

According to dual process accounts of persuasion (Chaiken, Liberman, \& Eagly, 1989; Petty \& Cacioppo, 1986; for an overview see Chaiken \& Trope, 1999), persuasive messages are processed in one of two modes: systematically or heuristically. With systematic processing, attention is paid to particular details in message content. With heuristic processing, attention is paid to surface features of the message (e.g., the expertise of the message source). These surface features can work as heuristic cues to facilitate persuasion. As mentioned in Chapter 1, it has been argued that under conditions of heuristic processing the positive infor- 
mation in gain-framed messages operates like a heuristic cue to facilitate persuasion, rendering gain-framed messages more persuasive than loss-framed messages. Under conditions of systematic processing, on the other hand, loss-framed messages might be more persuasive because of a negativity bias (Maheswaran \& Meyers Levy, 1990; Meyers Levy \& Maheswaran, 2004; Steward, Schneider, Pizarro, \& Salovey, 2003).

In Study 3.1, 3.2, and 4, positive affective reactions increased information acceptance and attitude, suggesting that positive feelings can play a crucial role in the persuasion process. These results corroborate theoretical accounts in marketing and consumer psychology proposing that advertisements that evoke positive affect result in a positive attitude towards the ad, which in turn results in a positive attitude towards the advertised brand (Brown, Homer, \& Inman, 1998; MacKenzie, Lutz, \& Belch, 1986). However, this affect transfer effect might be limited to conditions of heuristic processing. After all, the university students who we used as participants in these studies, are not likely to be deeply concerned with distal threats such as cardiovascular disease and hypertension. Therefore, the participants in these studies might have been inclined to process the framed information heuristically and positive affect might have operated like heuristic cues (see Maheswaran \& Meyers Levy, 1990; Meyers Levy \& Maheswaran, 2004; Steward, Schneider, Pizarro, \& Salovey, 2003). Thus, the results found in the present research with regards to positive affect might be the result of heuristic processing of the framed materials and it is currently unclear whether similar results would have been found under conditions of systematic processing. A limitation of this research, then, is that we can not rule out this alternative explanation.

In Study 5, 6 and 7 we found evidence to suggest that self-efficacy moderates message-framing effects. Again, however, it is unclear whether mode of information processing might have influenced these results. It might be argued that self-efficacy to behave in a healthy way can increase recipients' motivation to pay attention to health-promoting information. After all, for people with low self-efficacy, information about a healthy behaviour which they feel they cannot perform may seem irrelevant and they may be unlikely to pay much attention to it. People with high self-efficacy may have been far more motivated to process the information in an effortful way. For those participants, loss-framed information may have been more persuasive because under conditions of systematic processing, negative information may be more influential than equivalent positive information (Maheswaran \& Meyers Levy, 1990; Meyers Levy \& Maheswaran, 2004).

Lastly, mode of processing might have played an important role in Study 8.1 and 8.2. In these experiments, participants were more persuaded by gain- versus loss-framed information but particularly when the information was of a low-threat content. We hypothesized that congruence between information 
content and information frame was responsible for these results, but it is also possible that personal involvement (and hence the motivation to engage in effortful processing) was generally low in these participants, causing gain-framed information to be more persuasive than loss-framed information, especially in the low-threat condition. In the high-threat condition, on the other hand, the serious nature of the depicted consequences of unhealthy behaviour might have increased participants' personal involvement. This way, they might have relied on positive cues to a lesser extent. Thus, the high-threat information might have increased personal involvement, similar to Maheswaran and Meyers-Levy's (1990) information about participants' susceptibility (see Chapter 1). Perhaps as a result of this, the advantage of gain-framed information was reduced in the high-threat condition. Thus, the mode-of-processing hypothesis offers an alternative explanation for our results that we cannot rule out. Future research investigating the role of affect in framing effects, the influence of self-efficacy or framing effects in high- and low-threat information should pay specific attention to systematic and heuristic processing as alternative explanations. One could, for instance, provide participants with framed messages that vary in argument strength. When framed messages with strong arguments are more persuasive than framed messages with weak arguments, this offers an indication that participants engaged in systematic processing, paying attention to details in message content (cf. Updegraff, Sherman, Luyster, \& Mann, 2007). If Maheswaran and Meyers-Levy's (1990) reasoning is correct, than an advantage of loss-framed information over gain-framed information would co-occur with an advantage of high-quality arguments over low-quality arguments. Another possibility would be to decrease participants' potential for systematic processing, for example by giving them limited time to process the information. This way, participants would be forced to process the information heuristically and one could hypothesize that gain-framed information would thus be more persuasive.

In sum, a limitation of the present research is the fact that we cannot rule out that our effects were due to differences in heuristic and systematic processing. Future studies should further investigate the effects of positive and negative affective reactions on persuasion, the moderating role of self-efficacy and message threat under conditions of heuristic and systematic processing.

\section{Defensive processes}

The hypothesis that self-efficacy moderates message-framing effects relies on the assumption that self-efficacy decreases the need to engage in defensive processing of health-promoting information. Defensive processing, in turn, makes it less likely that threatening loss-framed information enhances persuasion. However, from the results of Study 5, 6 and 7, in which we investigated the influence of self-efficacy on the effects of framed messages, it is not entirely clear that the proposed defensiveness was in fact responsible for the found inter- 
action between framing and self-efficacy. In Study 5, no assessment of defensive reactions was included. In Study 6 and 7, we used information acceptance as an assessment of participants' defensive reactions. However, there are various other measures that can be used to assess defensive reactions such as avoidance and fatalism (see for instance Rippetoe \& Rogers, 1987) or more direct assessments, like the number of counter-arguments generated by recipients (Keller \& Block, 1999) or reading time (Reed \& Aspinwall, 1998). Although the results of a recent meta-analysis indicate that information acceptance is a reliable measure of defensive reactions (Good \& Abraham, 2007), by using information acceptance scores only we may have overlooked other important defensive processes. It should also be noted that obtaining similar results using a different experimental manipulation to induce or decrease defensiveness might provide stronger evidence that the effects of framing are different under conditions of high and low defensiveness. Recent research into self-affirmation shows, for instance, that affirming participants' self-worth can decrease the need to process healthpromoting information in a self-serving and defensive manner (Harris \& Napper, 2005; Sherman, Nelson, \& Steele, 2000; see Good \& Abraham, 2007). With regards to message framing we would hypothesize that gain- and loss-framed messages are not differently persuasive under normal conditions but that loss-framed information is more persuasive when recipients are self-affirmed and as a result are less motivated to engage in defensive processing. One other way to investigate whether defensive processes are important in message-framing effects is to investigate whether loss-framed messages trigger more defensive avoidance than gain-framed messages. Recently, studies using event-related-potential paradigms have been employed to investigate defensive avoidance in response to threatening health messages (cf. Kessels, Jansma, \& Ruiter, submitted). If such a study could show that loss-framed information is more strongly avoided than gainframed information this could also offer further proof that defensive reactions are important in message-framing effects. Thus, to obtain stronger evidence for our hypothesis future research could investigate the interactive effects of message framing and self-affirmation or could test whether gain- and loss-framed messages trigger different levels of defensive avoidance.

\section{Implications for research}

While discussing limitations and remaining questions above, we have suggested several possibilities for future message-framing research. We have recommended that affective reactions be operationalized more specifically, we have proposed that future research should pay specific attention to the possibility that framing effects are influenced by mode of information processing, and we have suggested that researchers may further inspect the hypothesis that defensive 
processing influences framing effects. Below, we discuss two other important implications for message-framing research: a) integrating mediation and moderation analyses, and b) disentangling the processes that lead to persuasion.

\section{Mediated moderation? Or moderated mediation?}

As we have seen above, both mediation and moderation analyses can foster our understanding of message-framing effects. What has been lacking in the studies that are reported in the present thesis, is an integrated model that can explain both mediation and moderation effects. Based on Experiments 3.1 and 3.2, we have proposed the model in Figure 9, in which gain-framed information leads to positive affective reactions, which in turn increase information acceptance and attitude, and loss-framed information leads to negative affective reactions, which influences intention. Based on Study 5, 6 and 7 it might be warranted to add self-efficacy to the model. Perhaps self-efficacy moderates the relationship between negative affect and intention. Based on studies investigating the effects of fear-arousing messages (Ruiter, Abraham, \& Kok, 2001; Witte, 1992; 1994) it might be expected that negative affect only leads to a higher intention when people feel they are capable of performing the recommended behaviour. In that case a moderated mediation could best explain and integrate the findings in the present thesis: loss-framed information leads to stronger negative affective reactions than gain-framed information, and the negative affective reactions lead to a stronger intention, but only when people have high self-efficacy. When people have low self-efficacy, negative affect does not lead to stronger intentions.

One other possibility is that self-efficacy influences the relationship between framing and negative affect. A recent study investigating the effects of selfaffirmation showed that recipients of health-promoting information who were self-affirmed reported stronger negative feelings as a result of the information than recipients who were not self-affirmed (Harris \& Napper, 2005). Thus, it seems possible that when people process health-promoting information in a defensive way they try to ignore or repress negative feelings. When they are not motivated to process the information defensively because of an affirmed sense of self, health-promoting information might be more likely to arouse strong negative affective reactions. High self-efficacy could be another factor that influences the extent to which health-promoting information evokes negative affective reactions. If this is the case, then our model should be adapted to include a mediated moderation, in which loss-framed information leads to stronger negative affective reactions, but especially in people with high self-efficacy, and negative affective reactions lead to stronger intentions. Future research should investigate the exact relations between framing, self-efficacy, positive affect, negative affect and persuasive outcome measures. More in general, the message-framing literature would benefit if researchers would seek to construct comprehensive theoretical models capable of integrating findings. 
Figure 9 Path model showing positive affect as a predictor of information acceptance and attitude, and negative affect as a predictor of intention

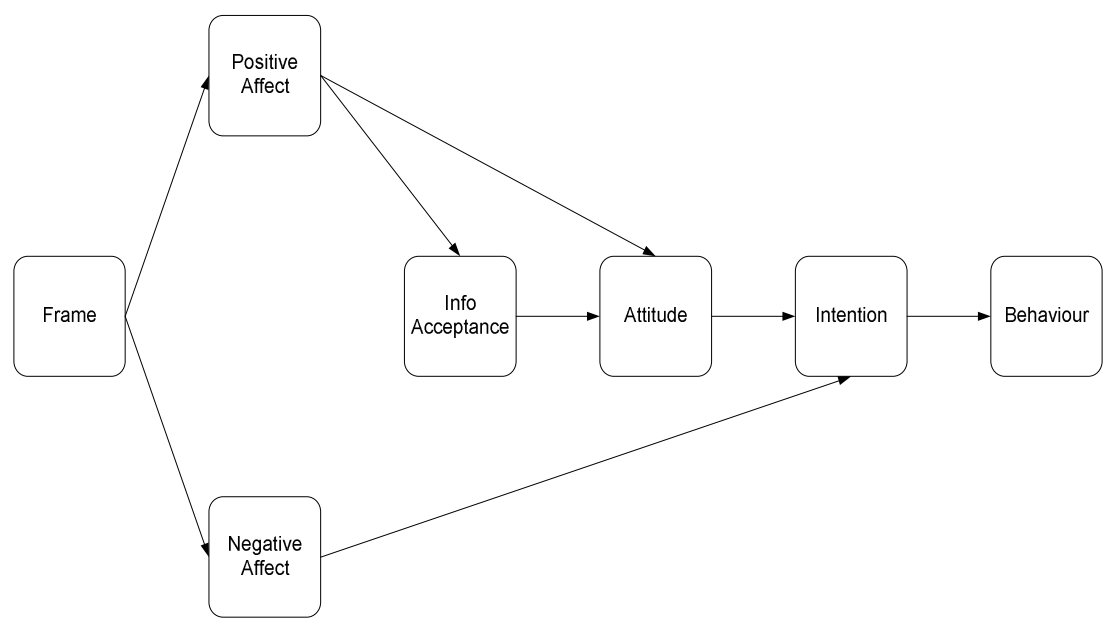

\section{Processes of persuasion}

Throughout this thesis, we refer to framed messages as 'healthpromoting' information or 'persuasive' messages and propose that their aim is to 'encourage' or 'motivate' people to engage in healthy behaviour. But what exactly do we mean by these terms? And when can we say that our efforts are 'effective' or 'persuasive'? Our findings that information acceptance, attitude and intention can be affected differently by message framing imply that persuasion is a dynamic process with several relevant steps. Traditionally, however, it has not been perceived as such, which we will outline below.

According to Green and Kreuter (1999), the aim of health education is to stimulate people to voluntarily behave in a healthful way. We agree with the idea that changing behaviour is the ultimate goal of health-education efforts. Therefore, we have included follow-up measures of actual health behaviour to investigate the effectiveness of framed messages in Study 2, 3.2 and 7 (whenever we use the term effectiveness, this refers to significant effects on behaviour). Green and Kreuter are not very clear, however, as to how these changes in behaviour are supposed to occur. Presumably, these changes can occur when people are motivated, but Green and Kreuter do not specify which processes can lead to motivation and under which circumstances motivation leads to behaviour. As noted above, recent meta-analytic findings suggest that message framing does not affect psychological and behavioural outcomes in a consistent way (O'Keefe \& Jensen, 2006; 2007). To shed some light on these inconclusive findings, it is 
worthwhile to focus on the process through which framing exerts its influence, rather than only on the outcomes of message-framing effects. Therefore, besides investigating the effects of framing on behaviour, we assessed whether a range of psychological variables could be influenced by message framing.

Our focus on psychological variables brings us closer to O'Keefe's (1990) definition of persuasion. According to this definition (O'Keefe, 1990, page 17) persuasion constitutes 'a successful, intentional effort at influencing another's mental state through communication in a circumstance in which the persuadee has some measure of freedom.' Other definitions are possible, of course, but O'Keefe's definition of persuasion seems representative of how the concept is used in the literature. Normally, the effect of different variables is assessed using an assessment of recipients' mental state, usually attitude, as the outcome measure. Assessing attitude as the only outcome measure of persuasion experiments poses a potential problem, however. After all, it is possible to hold a positive attitude towards for example physical activity, while still being unconvinced of its personal relevance. As Weinstein (1988) points out, accepting the personal relevance of a health message is a critical step in the precaution adoption process. For this reason, assessing participants' intention offers valuable extra information over assessing only attitude. Thus, a more dynamic approach to the study of persuasion might be in order. In this approach, persuasion is still considered as a change in one's mental state, but a person's mental state is no longer considered as a monolithic concept with attitude as its most likely exponent. Instead, persuasion might be seen as a process in which information acceptance, attitude, intention and behaviour are relevant steps. The results of Study 2 show that message framing can have an effect on intention, but not on information acceptance, attitude, and behaviour, whereas the results of Study 3.2 show that framing can affect information acceptance and attitude, but not intention. Furthermore, the results of Study 8.2 show that attitude and intention can sometimes be only weakly correlated. These findings suggest that several psychological processes interact to produce persuasion. Future research should investigate whether a more dynamic conceptualization of persuasion can yield more insight into the processes that are necessary for health-promoting messages to be effective.

\section{Sequential effects of different moderators}

The results of the studies reported in the present thesis suggest that both threat and self-efficacy can moderate message-framing effects. It remains unclear, however, whether threat and self-efficacy can moderate framing effects at the same time or whether they exert their influence in a sequential fashion. In their review of the fear-appeal literature, Ruiter and colleagues (2001) propose that threat recognition prompts action contemplation, whereas perceptions of efficacy and feasibility determine the kind of action that will be undertaken. Ef- 


\section{Framing Health Communication Messages}

ficacy and feasibility perceptions are therefore more proximal determinants of action (see also Rippetoe \& Rogers, 1987; Schwarzer, 1992). While threat perception may contribute to precautionary motivation by prompting consideration of outcome expectancies, perceptions of response- and self-efficacy may determine intention formation and subsequent action. Thus, one could hypothesize a sequential model in which message-framing effects are first influenced by perceived threat and second by self-efficacy. Future research could investigate whether threat and self-efficacy influence message-framing effects simultaneously or sequentially.

\section{Implications for practice}

As mentioned throughout the previous chapters, recent meta-analytic findings suggest that message framing does not affect psychological and behavioural outcomes in a consistent way (O'Keefe \& Jensen, 2006; 2007). Furthermore, the present thesis shows that, where main effects of framing were found at all, they were either small (Study 2) or limited to information acceptance and attitude (Study 3.1, 3.2, 8.1 and 8.2). Thus, it is difficult to arrive at clear and definitive recommendations for health-education practitioners who wonder how they should frame their messages. Two points could be made, however. First, gain-framed information might be used to responsibly increase levels of information acceptance. Second, interactions between framing and other relevant variables suggest that information can be adjusted to individual recipients' psychological attributes, such as self-efficacy, which is known as 'psychological tailoring' (Latimer, Katulak, Mowad, \& Salovey, 2005). We will outline these possibilities below.

\section{Increasing information acceptance}

The research that is presented in this thesis shows that, to increase the extent to which recipients perceive health-education messages as believable, relevant, and interesting, these messages might be better framed in terms of gains than in terms of losses. This recommendation has specific significance in an online context. Given the growing practice to use the Internet as a medium for health-promoting information and the importance of increasing people's exposure to this information (i.e., drawing attention and keeping recipients interested; see for instance Crutzen et al., 2008) information acceptance might be of critical importance. After all, people seeking information on the Internet are most likely to select information that seems relevant, interesting, and trustworthy. 


\section{Psychological tailoring}

Computer tailoring is a promising cost-effective health education technique that enables the development of individualized messages and is able to reach large populations against relatively low costs per person (Brug, Oenema, \& Campbell, 2003; De Vries \& Brug, 1999; Rimer \& Kreuter, 2006). Computer tailoring uses computer technology that adapts health education strategies and information to a specific person by addressing characteristics, circumstances, beliefs, motivation and behaviour unique for this individual and derived from an individual assessment (De Vries \& Brug, 1999; Kreuter, Farrell, Olevitch, \& Brennan, 2000). The effect of tailoring information to personal characteristics on health-related decision making is well documented (see for instance Noar, Benac, \& Harris, 2007) but tailoring the style or format of health-education messages to more general psychological characteristics is less systematically investigated (Latimer et al., 2005). Preliminary studies have shown that tailoring health-promoting information to individual differences in relatively stable psychological characteristics, such as need for cognition (Cacioppo, Petty, Feinstein, \& Jarvis, 1996) and need for affect (Maio \& Esses, 2001) may be more effective than generic health-messages. Need for cognition refers to an individual's tendency to engage in, and enjoy, effortful cognitive activities (see above). Need for affect refers to an individual's motivation to approach emotion-inducing situations. Recently, the results of three experiments showed that persuasive messages that were cognition-based (providing the recipient with logical arguments) were more persuasive for recipients with a high need for cognition, whereas affect-based messages (emphasizing the affective consequences of complying with message recommendations) were more persuasive for recipients with a high need for affect (Haddock, Maio, Arnold, \& Huskinson, 2008). Similarly, Mann and colleagues (2004) have suggested that framing can be used to tailor healthpromoting information to individual differences in approach and avoidance orientation. Since empirical studies have shown that for recipients with a predominant approach orientation, gain-framed information was more persuasive than loss-framed information whereas the reverse was true for recipients with a predominant avoidance orientation, it has been suggested that messages might be gain- or loss-framed to be congruent with recipients' approach/avoidance orientation (Gerend \& Shepherd, 2007; Mann, Sherman, \& Updegraff, 2004; Sherman, Mann, \& Updegraff, 2006). Because Study 4 failed to replicate the interaction between framing and approach/avoidance orientation, we propose that more research is needed to foster our knowledge of this congruency effect. Based on the results of Study 5, 6 and 7, however, we can suggest another possibility for psychological tailoring: providing recipients who are high in selfefficacy with loss-framed information and providing recipients' who are low in self-efficacy with gain-framed or mixed gain- and loss-framed messages. We hasten to say that, in light of the limitations of these studies discussed above, 
more research is needed to corroborate our findings. If future research dealing with these limitations suggests that health-promoting information can be framed to match recipients' self-efficacy levels, this could be an interesting possibility for health-education professionals.

\section{Conclusion}

The effects of framing health-promoting messages can be better understood by investigating underlying mechanisms of framing effects and the circumstances under which gain- versus loss-framed messages are more persuasive. The present research shows that gain-framed information can result in positive affective reactions, which in turn can contribute to greater information acceptance and greater attitude change. Loss-framed information on the other hand can result in negative affective reactions, which in turn can lead to stronger intentions. Also, we found that self-efficacy can influence the results of gain- and loss-framed messages. In particular, for recipients with high self-efficacy lossframed messages are more persuasive whereas for recipients with low selfefficacy gain- and loss-framed messages are equally persuasive. Lastly, gain frames were found to be more persuasive for low-threat information whereas findings were inconclusive for high-threat information. All in all, these results suggest that a) gain frames can be used to increase information acceptance, b) loss-framed information can be used when recipients have high self-efficacy, and c) gain-framed information can be used when the information has a low-threat content. 


\section{Footnotes}



${ }^{1}$ The plural 'we' is used in this thesis because the reported work resulted from collaboration with my supervisors and co-authors.

${ }^{2}$ For reasons of brevity, we focus on the similarities between Chaiken, Liberman, and Eagly's (1989) heuristic systematic model (HSM) and Petty and Cacioppo's (1986) elaboration likelihood model (ELM) instead of on the differences. In the same vein, we use the terms systematic and heuristic processing (HSM) as similar to the terms central and peripheral processing (ELM).

${ }^{3}$ Chapters 2-8 are based on papers that have been published or submitted for publication. Each chapter can be read independently of the other chapters, but as a result of this, the reader may encounter similarities among parts of this thesis.

${ }^{4}$ This chapter was based on: Van 't Riet, J., Ruiter, R. A. C., Werrij, M. Q., \& De Vries, H. (submitted). Investigating Message-Framing Effects in the Context of a Tailored Intervention Promoting Physical Activity.

${ }^{5}$ This chapter was based on: Van 't Riet, J., Ruiter, R. A. C., Werrij, M. Q., Candel, M. J. J. M., \& De Vries, H. (submitted). Distinct Pathways to Persuasion: The Role of Affect in Message-Framing Effects.

${ }^{6}$ In addition to this one-item measure, we included 4 items to assess consumption of salty products in the past week and used these to construct a saltconsumption index (cf. Parkington \& Roussos, 2008). Using this index in the analyses yielded similar results and identical conclusions.

${ }^{7}$ When $\chi^{2}<\mathrm{df}$, as is the case with the model in Figure 3.1, the CFIvalue is 1.000 and the RMSEA-value is .000 . Note that this does not necessarily mean that model fit is perfect (Kline, 2005). It does, however, indicate excellent model fit.

${ }^{8}$ Another point that could be made concerns the fact that two of the positive affect items used in Study 3.2, 'optimistic' and 'content', do not cover purely affective states but might also capture cognitive reactions. Therefore, we repeated the analyses excluding these items from the positive affect measure. This yielded similar results and identical conclusions.

${ }^{9}$ This chapter was based on: Van 't Riet, J., Ruiter, R. A. C., Werrij, M. Q., \& De Vries, H. (submitted). What Difference Does a Frame Make? Investigating the Influence of Affective Reactions to Skin Cancer Detection Messages.

${ }^{10}$ This chapter was based on: Van 't Riet, J., Ruiter, R. A. C., Werrij, M. Q., \& De Vries, (2008). The Influence of Self-Efficacy on the Effects of Framed Health Messages. European Journal of Social Psychology, 38, 800-809.

${ }^{11}$ This chapter was based on: Van 't Riet, J., Ruiter, R. A. C., Werrij, M. Q., \& De Vries, (in press). Self-Efficacy Moderates Message-Framing Effects: The Case of Skin-Cancer Detection. Psychology and Health.

${ }^{12}$ A second experimental manipulation was introduced after participants read the framed communication, but before they filled in the outcome measures. In the original design of the experiment, we aimed to test the interaction between 
self-efficacy and message framing, using a self-efficacy manipulation: half of the participants received self-efficacy enhancing information after they read the framed message, and half of the participants received no such information after reading the framed message. This self-efficacy manipulation was thus orthogonally crossed with the framing manipulation. Analyses revealed, however, that the self-efficacy manipulation did not succeed in raising self-efficacy levels. Furthermore, this manipulation did not yield any results of interest, alone or in combination with frame or baseline self-efficacy, $p \mathrm{~s}>.11$. For this reason, the manipulation will not be discussed further in this paper.

${ }^{13}$ This chapter was based on: Van 't Riet, J., Ruiter, R. A. C., Smerecnik, C., \& De Vries, (submitted). Examining the Influence of Self-Efficacy on Message-Framing Effects: Reducing Salt Consumption in the General Population.

${ }^{14}$ This chapter was based on: Van 't Riet, J., Ruiter, R. A. C., Werrij, M. Q., Candel, M. J. J. M., \& De Vries, (submitted). Investigating the Effects of Message Framing in High- and Low-Threat Information. 


\section{References}



Aaker, J. L., \& Lee, A. Y. (2001). "I" seek pleasures and "we" avoid pains: The role of self-regulatory goals in information processing and persuasion. Journal of Consumer Research, 28, 33-49.

Aiken, L. S., \& West, S. G. (1991). Multiple regression: Testing and interpreting interactions. New York: Sage.

Ajzen, I. (2002a). Attitudes. In R. Fernandez Ballesteros (Ed.), Encyclopedia of psychological assessment (Vol. 1, pp. 110-115). London: Sage.

Ajzen, I. (2002b). Perceived Behavioral Control, Self-Efficacy, Locus of Control, and the Theory of Planned Behavior. Journal of Applied Social Psychology, 32, 665-683.

American Cancer Society. (2008a). Cancer facts and figures 2008. Atlanta: American Cancer Society.

American Cancer Society (2008b). Skin cancer prevention and early detection. Atlanta: American Cancer Society.

Apanovitch, A. M., McCarthy, D., \& Salovey, P. (2003). Using message framing to motivate HIV testing among low-income, ethnic minority women. Health Psychology, 22, 60-67.

Baer, J. S., Holt, C. S., \& Lichtenstein, E. (1986). Self-efficacy and smoking reexamined: Construct validity and clinical utility. Journal of Consulting and Clinical Psychology, 54, 846-852.

Bandura, A. (1986). Social foundations of thought and action. Englewood Cliffs: Prentice-Hall.

Bandura, A., \& Cervone, D. (1986). Differential engagement of self-reactive influences in cognitive motivation. Organizational Behavior and $\mathrm{Hu}$ man Decision Processes, 38, 92-113.

Banks, S. M., Salovey, P., Greener, S., \& Rothman, A. J. (1995). The effects of message framing on mammography utilization. Health Psychology, 14, 178-184.

Baron, R. M., \& Kenny, D. A. (1986). The moderator-mediator variable distinction in social psychological research: Conceptual, strategic and statistical considerations. Journal of Personality and Social Psychology, 51, 1173-1182.

Blanton, H., Van den Eijnden, R. J. J. M., Buunk, B. P., Gibbons, F. X., Gerrard, M., \& Bakker, A. (2001). Accentuate the negative: Social images in the prediction and promotion of condom use. Journal of Applied Social Psychology, 31, 274-295.

Blaustein, M. P., Zhang, J., Chen, L., \& Hamilton, B. P. (2006). How does salt retention raise blood pressure? American Journal of Physiology - Regulatory, Integrative and Comparative Physiology, 290, R514-523.

Bless, H., \& Schwartz, N. (1999). Sufficient and necessary conditions in dualprocess models. In S. Chaiken \& Y. Trope (Eds.), Dual process theories in social psychology. New York: Guilford Press. 
Bolman, C., \& De Vries, H. (1998). Psycho-social Determinants and Motivational Phases in Smoking Behavior of Cardiac Inpatients. Preventive Medicine, 27, 738-747.

Broemer, P. (2002). Relative effectiveness of differently framed health messages: The influence of ambivalence. European Journal of Social Psychology, 32, 685-703.

Broemer, P. (2004). Ease of imagination moderates reactions to differently framed health messages. European Journal of Social Psychology, 34, 103119.

Brown, S. P., Homer, P. M., \& Inman, J. J. (1998). A meta-analysis of relationships between ad-evoked feelings and advertising responses. Journal of Marketing Research, 35, 114-126.

Brug, J., Oenema, A., \& Campbell, M. (2003). Past, present, and future of computer-tailored nutrition education. American Journal of Clinical Nutrition, 77, 1028s-1034s.

Brug, J., Ruiter, R. A. C., \& Van Assema, P. (2003). The (ir)relevance of framing nutrition education messages. Nutrition and Health, 17, 9-20.

Cacioppo, J. T., \& Berntson, G. G. (1994). Relationship between attitudes and evaluative space: A critical review, with emphasis on the separability of positive and negative substrates. Psychological Bulletin, 115, 401-423.

Cacioppo, J. T., Petty, R. E., Feinstein, J. A., \& Jarvis, W. B. G. (1996). Dispositional differences in cognitive motivation: The life and times of individuals varying in need for cognition. Psychological Bulletin, 119, 197253.

Carey, C. (1994). Rhetorical Means of Persuasion. In I. Worthington (Ed.), Persuasion: Greek Rhetoric in Action (pp. 26-45). London: Routledge.

Carver, C. S., \& White, T. L. (1994). Behavioral inhibition, behavioral activation, and affective responses to impending reward and punishment: The BIS/BAS Scales. Journal of Personality and Social Psychology, 67, 319-333.

CBS (2006). Jaarboek onderwijs in cijfers 2006 [Yearbook education in numbers 2006]. The Hague: Centraal Bureau voor de Statistiek [Central Bureau for Statistics].

Cesario, J., Grant, H., \& Higgins, E. T. (2004). Regulatory fit and persuasion: transfer from "Feeling Right." Journal of Personality and Social Psychology, 86, 388-404.

Chaiken, S., Liberman, A., \& Eagly, A. H. (1989). Heuristic and systematic information processing within and beyond the persuasion context. In J. Uleman \& J. Bargh (Eds.), Unintended thought (pp. 212-252). New York: Guilford.

Chaiken, S., \& Trope, Y. (Eds.). (1999). Duall-process theories in social psychology. New York, NY: Guilford Press. 
Chang, C. (2005). The moderating influence of ad framing for ad-selfcongruency effects. Psychology and Marketing, 22, 955-968.

Cherubini, P., Rumiati, R., Rossi, D., Nigro, F., \& Calabro, A. (2005). Improving attitudes toward prostate examinations by loss-framed appeals. Journal of Applied Social Psychology, 35, 732-744.

Choi, W. S., Gilpin, E. A., Farkas, A. J., \& Pierce, J. P. (2001). Determining the probability of future smoking among adolescents. Addiction, 96, 313323.

Cohen, J. (1992). A power primer. Psychological Bulletin, 112, 155-159.

Conner, M., \& Norman, P. (Eds.). (2005). Predicting health behaviour. Maidenhead (UK): Open University Press.

Cook, N. R., Cutler, J. R., Obarzanek, E., Buring, J. E., Rexrode, K. M., Kumanyika, S. K., Appel, L. J., Whelton, P. K. (2007). Long term effects of dietary sodium reduction on cardiovascular disease outcomes: observational follow-up of the trials of hypertension prevention (TOHP). British Medical Journal, 334, 885.

Cox, A. D., Cox, D., \& Zimet, G. (2006). Understanding consumer responses to product risk information. Journal of Marketing, 70, 79-91.

Cox, D., \& Cox, A. D. (2001). Communicating the consequences of early detection: The role of evidence and framing. Journal of Marketing, 65, 91103.

Craig, C. L., Marshall, A. L., Sjostrom, M. et al. (2003). International physical activity questionnaire: 12-country reliability and validity. Medical Science of Sports and Exercise, 35, 1381-1395.

Crutzen, R., de Nooijer, J., Brouwer, W., Oenema, A., Brug, J., \& de Vries, N. K. (2008). Internet-delivered interventions aimed at adolescents: a Delphi study on dissemination and exposure. Health Education Research, 23, 427-439.

De Hoog, N., Stroebe, W., \& De Wit, J. B. F. (2005). The impact of fear appeals on the processing and acceptance of action recommendations. Personality and Social Psychology Bulletin, 31, 24-33.

De Hoog, N., Stroebe, W., \& De Wit, J. B. F. (2008). The processing of feararousing communications: How biased processing leads to persuasion. Social Influence, 3, 84-113.

De Vries, H., \& Backbier, E. (1994). Self-efficacy as an important determinant of quitting among pregnant women who smoke: The ø-Pattern. Preventive Medicine, 23, 167-174.

De Vries, H., \& Brug, J. (1999). Computer-tailored interventions motivating people to adopt health promoting behaviours: Introduction to a new approach. Patient Education and Counseling, 36, 99-105.

De Vries, H., Mesters, I., Van 't Riet, J., Willems, K. \& Reubsaet, A. (2006). Motives of Belgian Adolescents for Using Sunscreen: The Role of Ac- 
tion Plans. Cancer, Epidemiology, Biomarkers, and Prevention, 15, 360-1366.

De Vries, H., Mudde, A., Dijkstra, A., \& Willemsen, M. C. (1998). Differential Beliefs, Perceived Social Influences, and Self-Efficacy Expectations among Smokers in Various Motivational Phases. Preventive Medicine, 27, 681-689.

Department of Health (2004). At least five a week: Evidence of the impact of physical activity and its relationship to health. London, UK: Department of Health.

Detweiler, J. B., Bedell, B. T., Salovey, P., Pronin, E., \& Rothman, A. J. (1999). Message framing and sunscreen use: Gain-framed messages motivate beach-goers. Health Psychology, 18, 189-196.

DiClemente, C. C., Prochaska, J. O., \& Gilbertini, M. (1985). Self-efficacy and the stages of self-change of smoking. Cognitive Therapy and Research, 9, 181-200.

Dijksterhuis, A., \& Aarts, H. (2003). On wildebeests and humans: the preferential detection of negative stimuli. Psychological Science, 14, 14-18.

Dijkstra, A., \& De Vries, H. (1999). The development of computer-generated tailored interventions. Patient Education and Counseling, 36, 193-203.

Dijkstra, A., Roijackers, J., \& De Vries, H. (1998). Smokers in Four Stages of Readiness to Change. Addictive Behaviors, 23, 339-350.

Dillard, J. P., \& Meijnders, A. (2002). Persuasion and the structure of affect. In J. P. Dillard \& M. Pfau (Eds.), The persuasion handbook: Developments in theory and practice (pp. 309-327). Sage, California: Thousand Oaks.

Dillard, J. P., \& Peck, E. (2000). Affect and persuasion: Emotional responses to public service anouncements. Communication Research, 27, 38-68.

Dillard, J. P., Weber, K. M., \& Vail, R. G. (2007). The relationship between the perceived and the actual effectiveness of persuasive messages: A metaanalysis with implications for formative campaign research. Journal of Communication, 57, 613-631.

Donovan, R. J., \& Alleh, G. (2000). Positive versus negative framing of a hypothetical infant immunization: the influence of involvement. Health Education and Behavior, 27, 82-95.

Dutch Cancer Society (2006). Ultraviolet radiation and skin cancer. Amsterdam: Dutch Cancer Society.

Eagly, A. H., \& Chaiken, S. (1993). The psychology of attitudes. Fort Worth (TX): Harcourt Brace Jovanovich.

Edwards, A., Elwyn, G., Covey, J., Matthews, E., \& Pill, R. (2001). Presenting risk information: A review of the effects of framing and other manipulations on patient outcomes. Journal of Health Communication, 6, 61-82.

Etter, J. F. (2006). Internet-based smoking cessation programs. Internet Journal for Medical Information, 75, 110-116. 
Eysenbach, G. (2005). The law of attrition. Journal of Medical Internet Research, 7, e11.

Fagerstrom, K. O. (1978). Measuring degree of physical dependence to tobacco smoking with reference to individualization of treatment. Addictive Behaviors, 3, 235-241.

Fiske, S. T. (1980). Attention and weight in person perception: The impact of negative and extreme behavior. Journal of Personality and Social Psychology, 38, 889-906.

Forgas, J. P. (1995). Mood and judgment: the affect infusion model (AIM). Psychological Bulletin, 117, 39-66.

Forgas, J. P. (2007). When sad is better than happy: Negative affect can improve the quality and effectiveness of persuasive messages and social influence strategies. Journal of Experimental Social Psychology, 43, 513-528.

Franken, I. H. A., Muris, P., \& Rassin, E. (2005). Psychometric Properties of the Dutch BIS/BAS Scales. Journal of Psychopathology and Behavioral Assessment, 27, 25-30.

Fredrickson, B. L., \& Branigan, C. A. (2005). Positive emotions broaden the scope of attention and thought-action repertoires. Cognition and Emotion, 19, 313-332.

Fredrickson, B. L., \& Losada, M. F. (2005). Positive affect and the complex dynamics of human flourishing. American Psychologist, 60, 678-686.

Frijda, N. H. (1986). The emotions. New York, NY: Camebridge University Press.

Frijda, N. H. (2007). The laws of emotion. Mahwah, NJ.: Lawrence Erlbaum.

Gerend, M. A., \& Cullen, M. (2008). Effects of message framing and temporal context on college student drinking behavior. Journal of Experimental Social Psychology, 44, 1167-1173.

Gerend, M. A., \& Shepherd, J. E. (2007). Using message framing to promote acceptance of the human papillomavirus vaccine. Health Psychology, 26, 745-752.

Goldman, M. S., Boyd, G. M., \& Faden, V. (2002). College drinking, what it is, and what to do about it: A review of the state of the science. Journal of Studies on Alcohol, Supl 14, 1-250.

Good, A., \& Abraham, C. (2007). Measuring defensive responses to threatening messages: A meta-analysis of measures. Health Psychology Review, 1, 208-229.

Gray, J. A. (1990). Brain systems that mediate both emotion and cognition. Cognition and Emotion, 4, 269-288.

Green, L. W., \& Kreuter, M. W. (1999). Health promotion planning: An educational and environmental approach $\left(3^{\text {rd }}\right.$ ed.). Mountain View, CA: Mayfield. 
Gwaltney, C. J., Shiffman, S., Norman, G. J., Paty, J. A., Kassel, J. D., Gnys, M., et al. (2001). Does smoking abstinence self-efficacy vary across situations? Identifying context-specificity within the Relapse Situation Efficacy Questionnaire. Journal of Consulting and Clinical Psychology, 69, 516-527.

Haddock, G., Maio, G. R., Arnold, K., \& Huskinson, T. (2008). Should persuasion be affective or cognitive? The moderating effects of need for affect and need for cognition. Personality and Social Psychology Bulletin, 34, 769-778.

Harris, P. R., \& Napper, L. (2005). Self-affirmation and the biased processing of threatening health-risk information. Personality and Social Psychology Bulletin, 31, 1250-1263.

He, J., \& Whelton, P. K. (1999). Elevated systolic blood pressure and risk of cardiovascular and renal disease: overview of evidence from observational epidemiological studies and randomized controlled trials. American Heart Journal, 138, S211-S219.

Higgins, E. T. (1997). Beyond pleasure and pain. American Psychologist, 52, 1280-1300.

Hildebrandt, V., Ooijendijk, W., Stiggelbout, M., \& Hopman-Rock, M. (2004). Trendrapport bewegen en gezondheid 2002-2003 [Tendency report physical activity and health]. Amsterdam.

Homer, P. M., \& Yoon, S. g. (1992). Message framing and the interrelationships among ad-based feelings, affect, and cognition. Journal of Advertising, 21, 19-32.

Isen, A. M., Daubman, K. A., \& Nowicki, G. P. (1987). Positive affect facilitates creative problem solving. Journal of Personality and Social Psychology, 52, 1122-1131.

Isen, A. M., Nygren, T. E., \& Ashby, F. G. (1988). Influence of positive affect on the subjective utility of gains and losses: it is just not worth the risk. Journal of Personality and Social Psychology, 55, 710-717.

Jones, L. W., Sinclair, R. C., \& Courneya, K. S. (2003). The effects of source credibility and message framing on exercise intentions, behaviors and attitudes: An integration of the elaboration likelihood model and prospect theory. Journal of Applied Social Psychology, 33, 179-196.

Jones, L. W., Sinclair, R. C., Rhodes, R. E., \& Courneya, K. S. (2004). Promoting exercise behaviour: An integration of persuasion theories and the theory of planned behaviour. British Journal of Health Psychology, 9, 505-521.

Kahneman D. (2003). A perspective on judgment and choice: Mapping bounded rationality. American Psychologist, 58, 697-720.

Kahneman, D., \& Tversky, A. (1979). Prospect Theory. An analysis of decision under risk. Econometrica, 47, 263-291. 
Kahneman, D., \& Tversky, A. (1984). Choices, values, and frames. American Psychologist, 39, 341-350.

Kanouse, D. E. (1984). Explaining negativity biases in evaluation and choice behavior: Theory and research. In T. C. Kinnear (Ed.), Advances in consumer research (Vol. 11, pp. 703-708). Provo, UT: Association for consumer research.

Keller, P. A., \& Block, L. G. (1999). The effect of affect-based dissonance versus cognition-based dissonance on motivated reasoning and healthrelated persuasion. Journal of Experimental Psychology: Applied, 5, 302-313.

Kemper, H. C. G., Ooijendijk, W. T. M., \& Stiggelbout, M. (2000). Consensus over de Nederlands norm voor gezond bewegen [Consensus regarding the Dutch norm for healthy physical activity]. Tijdschrift voor de Sociale Gezondheidszorg, 78, 180-183.

Kessels, L. T. E., Jansma, B. M., \& Ruiter, R. A. C. (submitted). Visiospatial attention processes to threatening smoking pictures: Evidence for defensive processes.

Kiene, S. M., Barta, W. D., Zelenski, J. M., \& Cothran, D. L. (2005). Why Are You Bringing Up Condoms Now? The Effect of Message Content on Framing Effects of Condom Use Messages. Health Psychology, 24, 321-326.

Kline, R. B. (2005). Principles and practice of structural equation modeling. New York, NY: The Guilford Press.

Kreuter, M., Farrell, D., Olevitch, L., \& Brennan, L. (Eds.). (2000). Tailoring health messages: Customizing communication with computer technology. Mahwah, NJ.: Lawrence Erlbaum Associates.

Kuhberger, A. (1998). The influence of framing on risky decisions: a metaanalysis. Organizational Behavior and Human decision Process, 75, 23-55.

Latimer, A. E., Katulak, N. A., Mowad, L., \& Salovey, P. (2005). Motivating cancer prevention and early detection behaviors using psychologically tailored messages. Journal of Health Communication, 10, 137-155.

Latimer, A. E., Rench, T. A., Rivers, S. E., Katulak, N. A., Materese, S. A., Cadmus, L., et al. (2008a). Promoting participation in physical activity using framed messages: An application of prospect theory. British Journal of Health Psychology, 13, 659-682.

Latimer, A. E., Rivers, S. E., Rench, T. A., Kataluk, N. A., Hicks, A., Hodorowski, J. K., et al. (2008b). A field experiment testing the utility of regulatory fit messages for promoting physical activity. Journal of Experimental Social Psychology, 44, 826-832. 
Latimer, A. E., Salovey, P., \& Rothman, A. J. (2007). The effectiveness of gainframed messages for encouraging disease prevention behavior: is all hope lost? Journal of Health Communication, 12, 645-649.

Lau, R. R. (1985). Two explanations for negativity effects in political behavior. American Journal of Political Science, 29, 119-138.

Lazarus, R. S. (1991). Emotion and adaptation. New York, NY: Oxford University Press.

Lee, A. Y., \& Aaker, J. L. (2004). Bringing the frame into focus: the influence of regulatory fit on processing fluency and persuasion. Journal of Personality and Social Psychology, 86, 205-218.

Leventhal, H. (1970). Findings and theory in the study of fear communications. In L. Berkowitz (Ed.), Advances in experimental social psychology (Vol. 5, pp. 119-187). New York: Academic Press.

Levin, I. P., Schneider, S. L., \& Gaeth, G. J. (1998). All frames are not created equal: A typology and critical analysis of framing effects. Organizational Behavior and Human Decision Processes, 76, 149-188.

Liberman, A., \& Chaiken, S. (1992). Defensive processing of personally relevant health messages. Personality and Social Psychology Bulletin, 18, 669679.

Locke, E. A., \& Latham, G. P. (2002). Building a practically useful theory of goal setting and task motivation. A 35-year odyssey. American Psychologist, 57, 705-717.

MacKenzie, S. B., Lutz, R. J., \& Belch, G. E. (1986). The role of attitude toward the ad as a mediator of advertising effectiveness: A test of competing explanations. Journal of Marketing Research, 18, 318-331.

MacKinnon, D. P., Lockwood, C. M., Hoffman, J. M., West, S. G., \& Sheets, V. (2002). A comparison of methods to test mediation and other intervening variable effects. Psychological Methods, 7, 83-104.

Maheswaran, D., \& Meyers Levy, J. (1990). The influence of message framing and issue involvement. Journal of Marketing Research, 27, 361-367.

Maio, G. R., \& Esses, V. M. (2001). The need for affect: individual differences in the motivation to approach or avoid emotions. Journal of Personality, 69, 583-615.

Mann, T., Sherman, D., \& Updegraff, J. (2004). Dispositional Motivations and Message Framing: A Test of the Congruency Hypothesis in College Students. Health Psychology, 23, 330-334.

McCall, L. A., \& Ginis, K. A. M. (2004). The Effects of Message Framing on Exercise Adherence and Health Beliefs Among Patients in a Cardiac Rehabilitation Program. Journal of Applied Biobehavioral Research, 9, 122-135. 
Meyerowitz, B. E., \& Chaiken, S. (1987). The effect of message framing on breast self-examination attitudes, intentions, and behavior. Journal of Personality and Social Psychology, 52, 500-510.

Meyers Levy, J., \& Maheswaran, D. (2004). Exploring message framing outcomes when systematic, heuristic, or both types of processing occur. Journal of Consumer Psychology, 14, 159-167.

Millar, M. G., \& Millar, K. (2000). Promoting safe driving behavior: The influence of message framing and issue involvement. Journal of Applied Social Psychology, 30, 853-866.

Miller, S. B., Friese, M., Dolgoy, L., Sita, A., Lavoie, K., \& Campbell, T. (1998). Hostility, sodium consumption, and cardiovascular response to interpersonal stress. Psychosomatic Medicine, 60, 71-77.

Mongeau, P. (1998). Another look at fear arousing messages. In M. Allen \& R. Preiss (Eds.), Persuasion: Advances through meta-analysis (pp. 53-68). Cresskill, NJ.: Hampton Press.

Noar, S. M. (2007). An interventionist's guide to AIDS behavioral theories. Aids Care, 19, 392-402.

Noar, S. M., Benac, C. N., \& Harris, M. S. (2007). Does Tailoring Matter? MetaAnalytic Review of Tailored Print Health Behavior Change Interventions. Psychological Bulletin, 133, 673-693.

O'Keefe, D. J. (1990). Persuasion: Theory and research. Thousand Oaks, CA.: Sage.

O'Keefe, D. J. (2002). Guilt as a mechanism of persuasion. In J. P. Dillard \& M. Pfau (Eds.), The persuasion handbook: Developments in theory and practice (pp. 329-344). Thousand Oaks, CA.: Sage.

O'Keefe, D. J., \& Jensen, J. D. (2006). The advantages of compliance or the disadvantages of noncompliance? A meta-analytic review of the relative persuasive effectiveness of gain-framed and loss-framed messages. In C. S. Beck (Ed.), Communication yearbook 30 (Vol. 30, pp. 1-44). Mahwah, NJ: Lawrence Erlbaum Associates.

O'Keefe, D. J., \& Jensen, J. D. (2007). The relative persuasiveness of gainframed and loss-framed messages for encouraging disease prevention behaviors: A meta-analytic review. Journal of Health Communication, 12, 623-644.

Parkington, S. R., \& Roussos, S. (2008). Measuring salt consumption to guide behavior change in applied settings: a critical review. American Journal of Health Promotion, 23, 101-107.

Peeters, A., Barendregt, J. J., Willekens, F., Mackenbach, J. P., Al Mamun, A., \& Bonneux, L. (2003). Obesity in adulthood and its consequences for life expectancy: A life-table analysis. Annals of Internal Medicine, 138, 24-32. 
Perdue, B., \& Summers, J. O. (1986). Checking the success of manipulations in marketing experiments. Journal of Marketing Research, 23, 317-326.

Petty, R. E., \& Cacioppo, J. T. (1986). Elaboration likelihood model of persuasion. In L. Berkowitz (Ed.), Advances in Experimental Social Psychology (Vol. 19, pp. 124-205): Academic Press Inc.

Prochaska, J. O., \& DiClemente, C. (1983). Stages and processes of self-change of smoking: Toward an integrative model of change. Journal of Consulting and Clinical Psychology, 51, 390-395.

Prochaska, J. O., Velicer, W. F., Rossi, J. S., Goldstein, M. G., Marcus, B. H., Rakowski, W., et al. (1994). Stages of change and decisional balance for 12 problem behaviors. Health Psychology, 13, 39-46.

Quirin, M., Kazén, M., \& Kuhl, J. (submitted). When nonsense sounds happy or helpness: The Implicit Positive and Negative Affect Test (IPANAT).

Reed, B., \& Aspinwall, L. G. (1998). Self-Affirmation Reduces Biased Processing of Health-Risk Information. Motivation and Emotion, 2, 99-130.

Reinhart, A. M., Marshall, H. M., Feeley, T. H., \& Tutzauer, F. (2007). The persuasive effects of message framing in organ donation: The mediating role of psychological reactance. Communication Monographs, 74, 229255.

Rimer, B. K., \& Kreuter, M. W. (2006). Advancing Tailored Health Communication: A Persuasion and Message Effects Perspective. Journal of Communication, 56, S184-S201.

Rippetoe, P. A., \& Rogers, R. W. (1987). Effects of components of protection motivation theory on adaptive and maladaptive coping with a health threat. Journal of Personality and Social Psychology, 52, 596-604.

Rivers, S. E., Salovey, P., Pizarro, D. A., Pizarro, J., \& Schneider, T. R. (2005). Message framing and pap test utilization among women attending a community health clinic. Journal of Health Psychology, 20, 65-77.

Robinson, M. D., \& Clore, G. L. (2002). Belief and feeling: Evidence for an accessibility model of emotional self-report. Psychological Bulletin, 128, 934-960.

Rogers, R. W. (1975). A protection motivation theory of fear appeals and attitude change. The Journal of Psychology, 91, 93-114.

Rogers, R. W. (1983). Cognitive and physiological processes in fear appeals and attitude change: A revised theory of protection motivation. In J. T. Cacioppo \& R. E. Petty (Eds.), Social psychophysiology: A sourcebook (pp. 153-176). New York: Guilford.

Rothman, A. J., Bartels, R. D., Wlaschin, J., \& Salovey, P. (2006). The Strategic Use of Gain- and Loss-Framed Messages to Promote Healthy Behavior: How Theory Can Inform Practice. Journal of Communication, 56, s202s220. 
Rothman, A. J., Martino, S. C., Bedell, B. T., Detweiller, J. B., \& Salovey, P. (1999). The systematic influence of gain-and loss-framed messages on interest in and use of different types of health behavior. Personality and Social Psychology Bulletin, 25, 1355-1369.

Rothman, A. J., \& Salovey, P. (1997). Shaping perceptions to motivate healthy behavior: the role of message framing. Psychological Bulletin, 121, 319.

Rothman, A. J., Salovey, P., Antone, C., Keough, K., \& Drake Martin, C. (1993). The influence of message framing on intentions to perform health behaviors. Journal of Experimental Social Psychology, 29, 408-433.

Rugg, D. (1941). Experiments in wording questions: II. Public Opinion Quarterly, 5, 91-92.

Ruiter, R. A. C., Abraham, C., \& Kok, G. (2001). Scary warnings and rational precautions: A review of the psychology of fear appeals. Psychology and Health, 16, 613-630.

Ruiter, R.A.C., De Nooijer, J., Van Breukelen, G., Ockhuysen-Vermey, C.F., \& De Vries, H. (2008). Intended Coping Responses to Cancer Symptoms in Healthy Adults: The Roles of Symptom Knowledge, Detection Behavior, and Perceived Threat. Cancer Epidemiology, Biomarkers \& Prevention, 17, 818-826.

Ruiter, R. A. C., Kessels, L. T, Jansma, B. M., \& Brug, J. (2006). Increased attention for computer-tailored health communications: an event-related potential study. Health Psychology, 25, 300-306.

Ruiter, R. A. C., Kok, G., Verplanken, B., \& Van Eersel, G. (2003). Strengthening the persuasive impact of fear appeals: the role of action framing. Journal of Social Psychology, 143, 397-400.

Ruiter, R. A. C., Verplanken, B., De Cremer, D., \& Kok, G. (2004). Danger and fear control in response to fear appeals: The role of need for cognition. Basic and Applied Social Psychology, 26, 13-24.

Ruys, K. I., \& Stapel, D. A. (in press). The secret life of emotions. Psychological Science.

Sanchez Castillo, C. P., Seidell, J., \& James, W. P. (1987). The potential use of lithium as a marker for the assessment of the sources of dietary salt: cooking studies and physiological experiments in men. Clinical Science, 72, 81-86.

Schneider, T. R., Salovey, P., Apanovitch, A. M., Pizarro, J., McCarthy, D., Zullo, J., et al. (2001a). The effects of message framing and ethnic targeting on mammography use among low-income women. Health Psychology, 20, 256-266.

Schneider, T. R., Salovey, P., Pallonen, U., Mundorf, N., Smith, N. F., \& Steward, W. T. (2001b). Visual and auditory message framing effects on tobacco smoking. Journal of Applied Social Psychology, 31, 667-682. 
Schwarz, N., Bless, H., \& Bohner, G. (1991). Mood and persuasion: Affective states influence the processing of persuasive communications. In L. Berkowitz (Ed.), Advances in experimental social psychology (Vol. 24, pp. 161-199). New York: Academic Press.

Schwarz, N., \& Clore, G. L. (2003). Mood as Information: 20 Years Later. Psychological Inquiry, 14, 296-303.

Schwarzer, R. (Ed.). (1992). Self-efficacy: Thought control of action. Washington, DC: Hemisphere.

Schwarzer, R., \& Luszczynska, A. (2008). How to overcome healthcompromising behaviors: The health action process approach. European Psychologist, 2, 141-151.

Shen, L., \& Dillard, J. P. (2007). The influence of behavioral inhibition/approach systems and message framing on the processing of persuasive health messages. Communication Research, 34, 433-467.

Sherman, D. A. K., Nelson, L. D., \& Steele, C. M. (2000). Do messages about health risks threaten the self? Increasing the acceptance of threatening health messages via self-affirmation. Personality and Social Psychology Bulletin, 26, 1046-1058.

Sherman, D. K., Mann, T., \& Updegraff, J. A. (2006). Approach/avoidance motivation, message framing, and health behavior: Understanding the congruency effect. Motivation and Emotion, 30, 165-169.

Shiv, B., Britton, J. A., Payne, J. W., Mick, D. G., \& Monroe, K. B. (2004). Does elaboration increase or decrease the effectiveness of negatively versus positively framed messages? Journal of Consumer Research, 31, 199208.

Sinclair, R. C., Mark, M. M., \& Clore, G. L. (1994). Mood-related persuasion depends on (mis)attributions. Social Cognition, 12, 309-326.

Smerecnik, C. M. R., Mesters, I., De Vries, N. K., De Vries, H. (in preparation). How does acceptance of health messages about genetics influence preventive behaviour?

Smith, G. E. (1996). Framing in advertising and the moderating impact of consumer education. Journal of Advertising Research, 36, 49-64.

Sobel, M. E. (1982). Asymptotic confidence intervals for indirect effects in structural equation models. In S. Leinhardt (Ed.), Sociological methodology 1982 (290-312). Washington, DC: American Sociological Association.

Sproston, K., \& Primatesta, P. (2004). Health Survey for England 2003. London: The Stationery Office.

Steffen, V. J., Sternberg, L., Teegarden, L. A. \& Sheperd, K. (1994). Practice and persuasive frame: effects on beliefs, intention, and performance of a cancer self-examination. Journal of Applied Social Psychology, 24, 897-925. 
Stephenson, M. T., \& Witte, K. (1998). Fear, threat, and perceptions of efficacy from frightening skin cancer messages. Public Health Reviews, 26, 147174.

Sternberg, R. J. (1988). The psychologist's companion: A guide to scientific writing for students and researchers. Cambridge, UK: Cambridge University Press.

Steward, W. T., Schneider, T. R., Pizarro, J., \& Salovey, P. (2003). Need for Cognition Moderates Responses to Framed Smoking-Cessation Messages. Journal of Applied Social Psychology, 33, 2439-2464.

Strecher, V. J., DeVellis, B. M., Becker, M. H., \& Rosenstock, I. M. (1986). The role of self-efficacy in achieving health behavior change. Health Education Quarterly, 13, 73-91.

Tate, D. F., \& Zabinski, M. F. (2004). Computer and Internet applications for psychological treatment: update for clinicians. Journal for Clinical Psychology, 60, 209-20.

Tucker, J., Ellickson, P., \& Klein, D. J. (2002). Smoking cessation during the transition from adolescence to young adulthood. Nicotine and Tobacco Research, 4, 321-332.

Tversky, A., \& Kahneman, D. (1981). The framing of decisions and the psychology of choice. Science, 211, 453-458.

Tykocinski, O., Higgins, T., \& Chaiken, S. (1994). Message framing, selfdiscrepancies, and yielding to persuasive messages: the motivational significance of psychological situations. Personality and Social Psychology Bulletin, 20, 107-115.

Updegraff, J. A., Sherman, D. K., Luyster, F. S., \& Mann, T. L. (2007). The effects of message quality and congruency on perceptions of tailored health communications. Journal of Experimental Social Psychology, 43, 249-257.

Van 't Riet, J., Ruiter, R. A. C., Werrij, M. Q., Candel, M. J. J. M., \& De Vries, H. (submitted). Distinct Pathways to Persuasion: The Role of Affect in Message-Framing Effects.

Van 't Riet, J., Ruiter, R. A. C., Werrij, M. Q., \& De Vries, (2008). The Influence of Self-Efficacy on the Effects of Framed Health Messages. European Journal of Social Psychology, 38, 800-809.

Van 't Riet, J., Ruiter, R. A. C., Werrij, M. Q., \& De Vries, H. (in press). SelfEfficacy Moderates Message-Framing Effects: The Case of Skin Cancer Detection. Psychology and Health.

Van Assema, P., Martens, M., Ruiter, R. A. C., \& Brug, J. (2001). Framing of nutrition education messages in persuading consumers of the advantages of a healthy diet. Journal of Human Nutrition and Dietetics, 14, 435-442. 
Van den Hooven, C., Fransen, H., Jansen, E., \& Ocké, M. (2007). 24-uurs urineexcretie van natrium: Voedingsstatusonderzoek bij volwassen Nederlanders. Bilthoven: Rijksinstituut voor Volksgezondheid en Milieu.

Van der Pligt, J., Zeelenberg, M., van Dijk, W. W., de Vries, N. K., \& Richard, R. (1998). Affect, attitudes and decisions: Let's be more specific. European Review of Social Psychology, 8, 33-66.

Wegener, D. T., Petty, R. E., \& Klein, D. J. (1994). Effects of mood on high elaboration attitude change: The mediating role of likelihood judgments. European Journal of Social Psychology, 24, 25-43.

Weinstein, N. D. (1988). The precaution adoption process. Health Psychology, 7, 355-386.

Wendel-Vos, G. C. W. (2008). Hoeveel mensen zijn voldoende lichamelijk actief? [How many people are sufficiently physically active?]. Bilthoven, The Netherlands: RIVM.

WHO (2002). The world health report 2002. Reducing risks, promoting healthy life. Geneva: World Health Organization.

WHO (2003). Diet, nutrition and the prevention of chronic diseases. Geneva: World Health Organization.

Witte, K. (1992). Putting the fear back into fear appeals: The extended parallel process model. Communication Monographs, 59, 329-349.

Witte, K. (1994). Fear control and danger control: A test of the extended parallel process model (EPPM). Communication Monographs, 61, 113-134.

Witte, K., \& Allen, M. (2000). A meta-analysis of fear appeals: implications for effective public health campaigns. Health Education and Behavior, 27, 591-615.

Wong, C. O., \& McMurray, N. E. (2002). Framing communication: communicating the antismoking message effectively to all smokers. Journal of Community Psychology, 30, 433-447.

Wright, P., \& Weitz, B. (1977). Time horizon effects on product evaluation strategies. Journal of Marketing Research, 14, 429-443.

Zajonc, R. B. (1980). Feeling and thinking: Preferences need no inferences. American Psychologist, 35, 151-175.

Zajonc, R. B. (1984). On the Primacy of Affect. American Psychologist, 39, $117-$ 123.

Zhang, Y., \& Buda, R. (1999). Moderating effects of need for cognition on responses to positively versus negatively framed advertising messages. Journal of Advertising, 28, 1-15. 


\section{Appendices}





\section{Appendix A: Gain- and loss-framed messages in Study 2}

\section{Gain-framed message}

\section{Health and Physical Activity}

Being active improves your health. Most people already know this. But being active also has advantages that not everybody knows.

\section{Muscles and Bones}

For instance, did you know that, by being physically active, you can keep your muscles and bones healthy? This is especially important for people over 30, because muscles and bones tend to grow weaker over time. Also, being physically active keeps you limber and feeling energetic.

\section{A Healthy Heart}

Being sufficiently physically active increases your chance of a healthy and strong heart. A healthy heart is an important condition for a long and healthy life, so there is plenty of reason to be physically active.

\section{Cancer}

Being active can also reduce your chance of cancer. Research shows that active people have a much smaller chance of colon cancer, besides from lung cancer the most common form of cancer in The Netherlands. For women, being active also reduces the chance of breast cancer. And breast cancer is the most common form of cancer in women.

\section{Diabetes}

Research shows that being active is the best way to prevent diabetes. Diabetes is caused by a small layer of fat that surrounds the organs. This fat can do a lot of damage to your health. If you are sufficiently active, these fats will not bother you and you will have an improved chance of a healthy life.

\section{Being Active and Relaxed}

Active people experience less stress and are better able to deal with it when they do. In other words, they are more relaxed. Also, they feel younger, more energetic, and simply better. In short, being active can help you feel good.

Other advantages of being active:

\section{Slender}

When you are active, you burn a lot of calories. This can help you become more slender. 


\section{Stamina}

When you are active, you will be stronger and have better stamina, something to be proud of! In short, being sufficiently active has many advantages!

\section{Loss-framed message}

\section{Health and Physical Activity}

Being inactive increases your risk of disease. Most people already know this. But being inactive also has disadvantages that not everybody knows.

\section{Muscles and Bones}

For instance, did you know that, by being physically inactive, your muscles and bones deteriorate? This is especially important for people over 30, because muscles and bones tend to grow weaker over time. Also, being physically inactive makes you less limber and feeling less energetic.

\section{An Unhealthy Heart}

Being insufficiently physically active increases your risk of cardiovascular diseases. Cardiovascular diseases are the number one cause of death in The Netherlands, so there is plenty of reason to make sure that you are not physically inactive.

\section{Cancer}

Being inactive can also increase your risk of cancer. Research shows that inactive people have a much greater risk of colon cancer, besides from lung cancer the most common form of cancer in The Netherlands. For women, being inactive also increases the risk of breast cancer. And breast cancer is the most common form of cancer in women.

\section{Diabetes}

Research shows that being inactive is the most important cause of diabetes. Diabetes is caused by a small layer of fat that surrounds the organs. This fat can do a lot of damage to your health. If you are insufficiently active, these fats will threaten your health.

\section{Being Inactive and Tense}

Inactive people experience more stress and have more trouble dealing with it when they do. In other words, they are more tense. Also, they feel older, less energetic, and simply worse. In short, being inactive can make you feel bad. 
Other disadvantages of being inactive:

Fat

When you are inactive, you do not burn a lot of calories. This can make you become more fat.

\section{Stamina}

When you are inactive, you will be less strong and have worse stamina, not something to be particularly proud of! In short, being inactive has many disadvantages! 


\section{Appendix B: Gain- and loss-framed messages in Study 3.2}

\section{Gain-framed message}

\section{The Advantages of Little Salt}

According to a recent report by the Dutch Health Council eating little salt is a sensible thing to do. The healthy thing to do is to eat less than six grams of salt each day. Most people eat way too much salt. The Dutch Society of Consumers estimates that the Dutch eat 10 to 12 grams of salt per day on average.

\section{Hypertension}

Scientific research shows that eating little salt may contribute to a normal and healthy blood pressure. If the Dutch would start to eat less salt, the incidence of hypertension in the Netherlands would fall.

\section{Cutting back on salt is good for your health}

Cutting back on salt is the most important way to prevent hypertension, even more important than exercising or quitting smoking. Having a normal blood pressure decreases your chance of getting a stroke or a heart attack.

\section{Other advantages of cutting back on salt}

- It decreases the risk of stomach cancer

- It decreases the risk of osteoporosis

- It decreases the risk of renal stones

In short, cutting back on salt can improve your health dramatically. Take this chance, cut back on salt!

\section{Loss-framed message}

\section{The Dangers of Too Much Salt}

According to a recent report by the Dutch Health Council eating too much salt is dangerous. Eating more than six grams of salt each day entails many risks. Most people eat way too much salt. The Dutch Society of Consumers estimates that the Dutch eat 10 to 12 grams of salt per day on average.

\section{Hypertension}

Scientific research shows that a salty diet may lead to hypertension. If the Dutch continue to eat too much salt, the incidence of hypertension in the Netherlands will rise.

\section{Too much salt is bad for your health}

A salty diet is the most important cause of hypertension, even more important than lack of exercise or smoking. Hypertension can lead to a much greater risk of a stroke or a heart attack.

\section{Other disadvantages of too much salt}

- It increases the risk of stomach cancer

- It increases the risk of osteoporosis

- It increases the risk of renal stones 
In short, eating too much salt can damage your health seriously. Avoid these dangers, be careful with salt! 


\section{Appendix C: Gain- and loss-framed messages in Study 4}

Gain-framed message

Dear participant,

A healthy skin is very important. A healthy skin does a better job at protecting people against damaging UV-radiation from the sun. In addition, people with a healthy skin look much younger. That is why it is important to check your skin for changes once a month. You can do this inspection yourself, for instance in front of a mirror.

\section{Early detection}

When you check your skin for changes once a month, you can detect skin cancer in an early stage. This way, you can do something about it right away.

\section{Cure}

As many people already know, the chances of being cured are much greater if skin cancer is detected early. Skin cancer does not have to be fatal, and especially if you check your skin for changes, you have a greater chance of a healthy life. The sooner skin cancer is detected, the greater the chances of being cured.

\section{Recovery}

Early detection can provide the skin with the opportunity to recover without developing nasty scars. This is especially important for parts of the body that are frequently exposed to the sun, like the face.

\section{Feeling relaxed}

Checking your skin for changes can also give you a relaxed feeling. People who inspect their skin often feel more relaxed about their skin's health.

\section{Loss-framed message \\ Dear participant,}

An unhealthy skin can cause a lot of problems. An unhealthy skin does a poorer job at protecting people against damaging UV-radiation from the sun. In addition, people with an unhealthy skin look much older. That is why it is unwise not to check your skin for changes once a month. You can do this inspection yourself, for instance in front of a mirror. 


\section{Late detection}

When you do not check your skin for changes once a month, you might detect skin cancer in a late stage. This could have serious consequences.

\section{Fatal}

As many people already know, the chances of being cured are much smaller if skin cancer is detected late. Skin cancer can be fatal, and especially if you do not check your skin for changes you run a greater risk of an unhealthy life. The later skin cancer is detected, the greater the risk you will not recover.

\section{Scars}

If skin cancer is detected too late, you might not have the opportunity to recover without developing nasty scars. This is especially important for parts of the body that are frequently exposed to the sun, like the face.

\section{Feeling worried}

Failing to check your skin for changes can also give you a worried feeling. People who do not inspect their skin often feel more worried about their skin's health. 


\section{Appendix D: Gain- and loss-framed messages in Study 5}

Gain-framed message

Why is it good to quit smoking?

The number of Dutch people that has quit smoking is still increasing each year. Quitting smoking is the best way to save lives. In fact, if you stop smoking, you will experience benefits in the short- and the long run.

- Your risk of lung cancer or a brain hemorrhage will decrease. You should worry far less about your health than someone who smokes.

- If you quit smoking, you will have more energy and feel better.

- Quitting smoking improves your physical condition and stamina; when you are physical active, it will take you more time to get tired and you will pant less.

- Your clothes will no longer smell like tobacco smoke.

- $\quad$ Quitting smoking makes your teeth whiter.

- Besides from all that, you will save a lot of money if you quit smoking, money that you can now spend on other things

In short, it is clear that you will experience many benefits from quitting smoking and your life will be a lot healthier.

\section{Loss-framed message}

Why is it bad to continue smoking?

The number of Dutch people who smoke is still decreasing each year. Smoking is the most important cause of deadly diseases. In fact, if you continue smoking, you will experience costs in the short- and the long run.

- Your risk of lung cancer or a brain hemorrhage will increase. You should worry more about your health than someone who does not smoke.

- If you continue smoking, you will have less energy and feel worse.

- Smoking damages your physical condition and stamina; when you are physical active, it will take you less time to get tired and you will pant more.

- Your clothes will still smell like tobacco smoke.

- $\quad$ Smoking makes your teeth less white.

- Besides from all that, you will spend a lot of money on cigarettes, money that you can not spend on other things 
In short, it is clear that you will experience many costs from continuing smoking and your life will be a lot less healthy. 


\section{Appendix E: Gain- and loss-framed messages in Study 7}

\section{Gain-framed communication}

\section{The advantages of a low-salt diet}

According to a new report from the health council, it is wise to eat a low-salt diet. The best thing to do would be to eat less than 6 grams of salt per day. Most people, however, consume a lot more salt. Consumer organizations estimate that Dutch people eat 10 to 12 grams of salt per day on average.

\section{Good health}

If you eat healthy, exercise regularly and are not overweight, you have a greater change of a normal and healthy blood pressure. By decreasing your salt consumption, however, you can be even healthier.

- Scientific research shows that a low-salt diet contributes to a normal and healthy blood pressure.

- In fact, decreasing your salt intake is the best way to prevent hypertension.

- Even when your blood pressure is healthy right now, it is wise to watch your salt consumption.

\section{Healthy blood pressure}

Why is it important to have a healthy blood pressure?

- A healthy blood pressure prevents there being too much pressure on the sides of your veins.

- This will prevent cracks and holes in your veins and the leaking of blood. This means you will have a smaller chance of a stroke, which occurs as a result of blood leaking in the brain.

- Another advantage of a healthy blood pressure is that it accommodates the flow of blood to the heart; this decreases your chance of a heart attack.

- One last advantage of a healthy blood pressure is that it keeps your arteries healthy. It decreases arterioscleroses, which makes it easier to supply each part of your body with blood.

\section{Other advantages of a low-salt diet}

Now you know that a low-salt diet increases the chance of a healthy blood pressure. But a low-salt diet has additional advantages. Scientific research shows that a low-salt diet can decrease your chances of other afflictions:

- It decreases the chance of stomach cancer

- It decreases the chance of osteoporosis

- It decreases the chance of kidney stones and other diseases of the kidneys 
In short, eating less salt will benefit your health.

\section{Loss-framed communication}

\section{The disadvantages of a high-salt diet}

According to a new report from the health council, it is unwise to eat a high-salt diet. It is unhealthy to eat more than 6 grams of salt per day. Most people, however, consume a lot more salt. Consumer organizations estimate that Dutch people eat 10 to 12 grams of salt per day on average.

\section{Ill health}

If you eat unhealthy, do not exercise regularly and are overweight, you have a greater risk of a high blood pressure. By eating a lot of salt, however, you will be even unhealthier.

- Scientific research shows that a high-salt diet contributes to a high blood pressure.

- In fact, a high salt intake is the most important cause of hypertension.

- Even when your blood pressure is healthy right now, it is unwise to let your salt consumption get out of hand.

\section{High blood pressure}

Why is it dangerous to have a high blood pressure?

- With a high blood pressure, there is too much pressure on the sides of your veins.

- This will cause cracks and holes in your veins and the leaking of blood. This means you will have a greater risk of a stroke, which occurs as a result of blood leaking in the brain.

- Another disadvantage of a high blood pressure is that it obstructs the flow of blood to the heart; this increases your risk of a heart attack.

- One last disadvantage of a high blood pressure is that it damages your arteries. It increases arterioscleroses, which makes it harder to supply each part of your body with blood.

\section{Other disadvantages of a high-salt diet}

Now you know that a high-salt diet increases the chance of a high blood pressure. But a high-salt diet has additional disadvantages. Scientific research shows that a high-salt diet can increase your risk of other afflictions:

- It increases the risk of stomach cancer

- It increases the risk of osteoporosis

- It increases the risk of kidney stones and other diseases of the kidneys

In short, eating a lot of salt will endanger your health. 


\section{Appendix F: Self-efficacy enhancing information in Study 7}

\section{Eating less salt: very easy}

Did you know that it is very easy to reduce the amount of salt in your diet?

The following tips can help you with this:

- You could season your vegetables and salads with vinegar instead of salt.

- You can give your dinner more spice with ginger, garlic, or chili peppers.

- You could use wine in casseroles.

- You could use herbs such as basil, oregano, parsley, coriander, rosemary, or cinnamon to give flavour to your dinner.

You might not know that there are a lot of products that contain salt. But you can easily replace these products with low-salt products.

- Many cheeses and sausages contain a lot of salt. But there are also lowsalt cheeses and sausages. You could ask for this in your supermarket.

- Ketch-up and curry contain a lot of salt. If you apply the sauce next to your food on the plate, instead of over it, this will make sure that you eat less salt.

- $\quad$ Eating unsalted peanuts instead of salted peanuts is another way to eat less salt. It's as easy as that!

- With microwave dinners you can read about the amount of salt on the package and you can choose the product with the lowest amount of salt.

In short, there are plenty of ways to enjoy your food with less salt. Eating less salt is very easy. 


\section{Appendix G: High- and low-threat gain- and loss-framed messages in Study} 8.2

\section{High-threat, gain-framed communication}

\section{Alcohol}

Many people think that drinking a small amount of alcohol a day is good for your heart and your blood vessels. Not everyone knows, however, that alcohol only has benefits for men older than 40 and post-menopausal women. For students (like you) alcohol does not cnotribute to your health. It is not true that drinking a beer or a glass of wine once in a while is good for your heart.

On the contrary, it is very important NOT to drink TOO MUCH. Effectively, this means that women should not drink more than 14 glasses a week and men should not drink more than 21 glasses a week. NOT DRINKING TOO MUCH has several advantages.

\section{A better functioning brain}

One advantage of not drinking too much is that you are protecting your brain from the detrimental effects of alcohol. The day after a party, for instance, you will be much better able to remember what happened and you will not be suffering from black-outs or other disorders of the brain. You will have no problems with your memory. Both the short-term and the long-term memory will benefit from this, but especially your long-term memory is better of when you do not drink too much. The long-term memory happens to be very sensitive to alcoholinduced damage, even when it has been a long while since you drank. By not drinking too much you can protect your long-term memory.

\section{Graduating sooner}

Not drinking too much has several other advantages. Among other things it positively influences your study results. A recent study at the Radboud University of Nijmegen showed that students who do not drink too much alcohol receive better grades and graduate sooner. This makes sense, because people who do not drink to much have better long-term memory and can concentrate more easily.

\section{A healthy stomach and liver}

Another important advantage of not drinking too much alcohol is the fact that it is good for your stomach and liver. By not drinking to much, you can safe your stomach and liver from being affected. You can even avoid gastro-intestinal bleeding or cirrhosis of the liver. In short, you live a much healthier life and do not need to worry about the health of your stomach and liver. 


\section{Conclusion}

Not drinking too much has many advantages, both on the short-term (no blackouts, better concentration) and on the long term (a healthy stomach and liver). Make sure that you drink less than 14 glasses a week, or 21 for men.

\section{High-threat, loss-framed communication}

\section{Alcohol}

Many people think that drinking a small amount of alcohol a day is good for your heart and your blood vessels. Not everyone knows, however, that alcohol only has benefits for men older than 40 and post-menopausal women. For students (like you) alcohol does not cnotribute to your health. It is not true that drinking a beer or a glass of wine once in a while is good for your heart.

On the contrary, drink TOO MUCH is very dangerous. Effectively, this means that women should not drink more than 14 glasses a week and men should not drink more than 21 glasses a week. DRINKING TOO MUCH has several disadvantages.

\section{Braindamage}

One disadvantage of drinking too much is that your brain is suffering the detrimental effects of alcohol. The day after a party, for instance, you might not be able to remember what happened; this means you are suffering from a black-out, which is a disorder of the brain. Your memory is not working the way it should. Both the short-term and the long-term memory will be damaged, but especially your short-term memory is worse of when you drink too much. The long-term memory happens to be very sensitive to alcohol-induced damage, even when it has been a long while since you drank. By drinking too much you can damage your long-term memory.

\section{Graduating later}

Drinking too much has several other disadvantages. Among other things it negatively influences your study results. A recent study at the Radboud University of Nijmegen showed that students who drink too much alcohol receive worse grades and graduate later. This makes sense, because people who drink too much have poorer long-term memory and have more problems concentrating

\section{An unhealthy stomach and liver}

Another important disadvantage of drinking too much alcohol is the fact that it is bad for your stomach and liver. By drinking too much, you can severely damage your stomach and liver. You can even get gastro-intestinal bleeding or cirrhosis of the liver. In short, you live a much unhealthier life and need to worry about the health of your stomach and liver. 


\section{Conclusion}

Drinking too much has many disadvantages, both on the short-term (black-outs, poorer concentration) and on the long term (an unhealthy stomach and liver). Make sure that you do not drink more than 14 glasses a week, or 21 for men.

\section{Low-threat, gain-framed communication}

\section{Alcohol}

Many people think that drinking a small amount of alcohol a day is good for your heart and your blood vessels. Not everyone knows, however, that alcohol only has benefits for men older than 40 and post-menopausal women. For students (like you) alcohol does not cnotribute to your health. It is not true that drinking a beer or a glass of wine once in a while is good for your heart.

On the contrary, it is very important NOT to drink TOO MUCH. Effectively, this means that women should not drink more than 14 glasses a week and men should not drink more than 21 glasses a week. NOT DRINKING TOO MUCH has several advantages.

\section{You are safer}

One advantage of not drinking too much is that you are safer. When you are sober, you are better able to judge and master situations. Research shows that people who do not drink too much have less falling accidents and are less often involved in traffic accidents. By not drinking too much you have a smaller chance of ending up in a hospital or causing trouble for other people. Not just when you are driving a car, but also when you ride a bike, it is safer to be sober.

\section{Better immune system}

Not drinking too much alcohol has more advantages. For instance, alcohol has a positive effect on your metabolism. When you do not drink too much your body has no trouble breaking down alcohol. It is left with enough energy for the construction of proteins and for converting vitamins and minerals into active chemicals. Because of these vitamins, minerals and proteins, your body is able to protect itself from disease. So, not drinking too much alcohol improves your immune system.

\section{Staying thin more easily}

Another important advantage of not drinking too much alcohol is the fact that you can stay thin more easily. When your body breaks down the alcohol, this results in extra energy. One gram of alcohol can be responsible for 29 kilojoules ( 7 calories). And one glass of beer or wine can contain up to 10 grams of alcohol. Not drinking too much can help you to stay thin or lose weight more easily. 


\section{Conclusion}

Not drinking too much has many advantages, both on the short-term (fewer accidents, a stronger immune system) and on the long term (staying thin). Make sure that you drink less than 14 glasses a week, or 21 for men.

\section{Low-threat, loss-framed communication}

\section{Alcohol}

Many people think that drinking a small amount of alcohol a day is good for your heart and your blood vessels. Not everyone knows, however, that alcohol only has benefits for men older than 40 and post-menopausal women. For students (like you) alcohol does not cnotribute to your health. It is not true that drinking a beer or a glass of wine once in a while is good for your heart.

On the contrary, drink TOO MUCH is very dangerous. Effectively, this means that women should not drink more than 14 glasses a week and men should not drink more than 21 glasses a week. DRINKING TOO MUCH has several disadvantages.

\section{You are less safe}

One disadvantage of drinking too much is that you are less safe. When you have had too much to drink, you are less able to judge and master situations. Research shows that people who drink too much have more falling accidents and are more often involved in traffic accidents. By drinking too much you have a greater risk of ending up in a hospital or causing trouble for other people. Not just when you are driving a car, but also when you ride a bike, it is more dangerous to drink.

\section{A weaker immune system}

Drinking too much alcohol has more disadvantages. For instance, alcohol has a negative effect on your metabolism. When you drink too much your body has trouble breaking down the alcohol. It is left without enough energy for the construction of proteins and for converting vitamins and minerals into active chemicals. Because of the lack of these vitamins, minerals and proteins, your body is not able to protect itself from disease. So, drinking too much alcohol weakens your immune system.

\section{Having trouble staying thin}

Another important disadvantage of drinking too much alcohol is the fact that you can have more trouble staying thin. When your body breaks down the alcohol, this results in extra energy. One gram of alcohol can be responsible for 29 kilojoules ( 7 calories). And one glass of beer or wine can contain up to 10 grams of alcohol. Drinking too much can make staying thin or losing weight more difficult. 


\section{Conclusion}

Drinking too much has many disadvantages, both on the short-term (more accidents, a weaker immune system) and on the long term (more trouble staying thin). Make sure that you drink less than 14 glasses a week, or 21 for men. 



\section{Summary}

Health-promoting messages can be framed in terms of the gains associated with healthy behaviour, or the losses associated with unhealthy behaviour. Studies show inconsistent results with regard to which type of framing is more persuasive. One influential approach to the study of message framing has been to focus on the function of the recommended behaviour, making a distinction between behaviours that serve to prevent an illness (like exercising or quitting smoking) and behaviours that serve to detect an illness (like skin self-examination or obtaining a mammography). According to this view, people perceive diseaseprevention behaviours as relatively safe, because they minimize the chance of falling ill. In contrast, people perceive disease-detection behaviours as inherently risky because they entail the possibility of finding out that one is ill. Drawing from Prospect Theory, it has been suggested that gain-framed information is more persuasive when advocating disease-prevention behaviours, because gainframed information makes people risk-avers and thus more likely to engage in relatively safe disease-prevention behaviours. In contrast, loss-framed information is said to be more persuasive for disease-detection behaviours, because lossframed information makes people willing to take risks and thus more likely to engage in relatively risky disease-prevention behaviours. However, recent findings shed doubt on this reasoning. To provide a further test of this hypothesis, we conducted a study into the effects of gain- and loss-framed messages advocating physical activity, a prevention behaviour. The results of this study are reported in Chapter 2. Because no previous studies investigated the effects of gain- and lossframed messages in the context of a tailored intervention to promote physical activity, we first provided participants $(\mathrm{N}=787)$ with computer-generated tailored feedback concerning their physical activity levels. Next, we provided participants with gain- and loss-framed messages promoting physical activity and investigated whether gain- and loss-framed messages affected information acceptance, attitude, intention and behaviour differently. The results showed that gainframed messages resulted in stronger intentions to be physically active than lossframed messages. This did not result in a significant increase in actual physical activity, however, as measured by a three-month follow-up assessment. For information acceptance and attitude, a non-significant advantage of gain-framed messages was found. All effects had small effect sizes. These results suggest that, whereas gain-framed information might be more persuasive than lossframed information when it comes to promoting physical activity, the differences between gain- and loss-framed messages are likely to be small.

From the inconsistent findings in the literature and the results of the study reported in Chapter 2, it can be concluded that a distinction between pre- 
vention and detection behaviours is not sufficient to explain and understand message-framing effects. Instead, we argue that researchers can foster our understanding of message framing in two ways. First, it is important to investigate the mechanisms that underlie message-framing effects. Second, important insights can be gained by investigating the circumstances under which gain- or lossframe information is more persuasive. In line with this reasoning, Part 2 of this thesis (Chapters 3-4) is concerned with identifying underlying mechanisms of framing effects, hypothesizing that positive and negative affective reactions can mediate the effects of framing. Part 3 (Chapter 5-8) is concerned with identifying moderating variables that can influence the effects of framing. In Part 3, it is hypothesized that self-efficacy and message threat can moderate the effects of message framing.

In Chapter 3 and 4, we report the results of three studies that investigated the mediating role of positive and negative affective reactions on the effects of message framing. Previous research shows that when messages produce positive affect, this positive affect can lead to a positive attitude towards the message, and a positive attitude towards the position that is advocated in the message, an effect that is known as the 'affect transfer hypothesis'. In addition, negative affect can also contribute to persuasion, which can be seen, for instance, in the literature on fear appeals. We propose that, when positive affect as a result of gain-framed messages and negative affect as a result of loss-framed messages contribute equally to persuasion, it is likely that gain- and loss-framed messages are not differentially persuasive. This can account for the null-findings and inconsistent results that are typical for the message-framing literature. In Study 3.1 we provided 100 student participants with gain- and loss-framed information advocating physical activity. The results showed that gain-framed information resulted in higher levels of information acceptance than loss-framed information and this effect was mediated by positive affect. The results of Study 3.2, in which we provided 129 student participants with framed messages advocating a low-salt diet, showed that gain-framed information resulted in higher levels of information acceptance and attitude, an effect that was again mediated by positive affect. In addition, loss-framed information resulted in more negative affect than gain-framed information and negative affect increased participants' intention to engage in the healthy behaviour. In Study 4, these findings were replicated with one exception. In Study 4, positive affective reactions did not contribute to attitude. These results suggest that affect may be of great importance in the persuasion process and may be particularly helpful to explain the underlying mechanisms of message framing effects. The findings also suggest that gain- and loss-framed messages offer distinct pathways to persuasion. In particular, gainframed information may exert its effect on recipients through the persuading influence of positive affect, whereas loss-framed information may exert its effect on recipients through the motivating influence of negative affect. 
In Chapter 5, we examined the influence of self-efficacy to quit smoking on the effects of gain- and loss-framed anti-smoking messages among 539 adult smokers. Previous research shows that threatening health-promoting messages can be effective, provided that recipients feel they have the ability to cope with the threat. Because loss-framed messages have been found to evoke a greater sense of threat than gain-framed messages, a similar mechanism may be at work with regards to loss-framed messages. We propose that loss-framed messages may be effective, provided that recipients have high self-efficacy to engage in the recommended behaviour. The results of Study 5 showed that participants with high self-efficacy to quit smoking reported a stronger intention to quit smoking after receiving a loss-framed message than after receiving a gainframed or no message. For these participants receiving a gain-framed message did not result in a stronger intention than receiving no message. For participants with low self-efficacy there were no differences in intention between the gainframed message condition, loss-framed message condition, and control condition. These results suggest that self-efficacy can moderate the effects of message framing on persuasion.

In Chapter 6, we report on a study which aimed to replicate the moderating influence of self-efficacy. The results of Study 5 showed that self-efficacy can moderate the effects of framed messages advocating smoking cessation, a prevention behaviour. To increase the generalizability of our results, and because several researchers propose that framing effects are different in prevention versus detection behaviour, we used framed messages advocating skin self-examination, a detection behaviour, in Study 6. The results of this study, among 124 university students, showed that, for participants with high self-efficacy, a loss-framed message resulted in a higher intention to perform skin self-examination than a gain-framed message. For participants with low self-efficacy, there were no differences in intention between the gain- and loss-framed message conditions. Once again, these results suggest that self-efficacy levels play an important role in message-framing effects. For health communication strategies promoting the active detection of skin-cancer symptoms, messages stressing losses may be more effective than messages stressing gains, but only in persons with high selfefficacy.

The results of Study 5 and 6 suggest that self-efficacy can moderate the effects of framed messages. However, these studies were subject to two important limitations. First, they relied on observational assessments of self-efficacy instead of experimental manipulations, making it impossible to draw strong conclusions about the causality of the effect. Second, these studies did not include an assessment of actual health behaviour, making it impossible to assess behavioural effects. In Chapter 7, therefore, we investigated whether we could replicate the interaction between self-efficacy and framing, using an experimental manipulation of self-efficacy and a three week follow-up assessment of behav- 
iour. A total of 575 adults, recruited from an Internet-panel, took part in the study. Half of the participants received self-efficacy enhancing information, whereas the other half received no such information. After this self-efficacy manipulation, half of the participants received a gain-framed, and half of the participants received a loss-framed message promoting a low-salt diet. The results of a 3-week follow-up supported our hypothesis. Our results suggest that messages stressing losses may be more effective than messages stressing gains, but only in persons with high self-efficacy. In Study 7, however, in contrast to Study 5 and 6 , we did not find the hypothesized interaction on the outcome measure intention.

In Chapter 8, we report on a study that examined whether the effect of message frame is different for low- versus high-threat information. The hypothesis was that a gain-frame might be more persuasive for low-threat information whereas a loss frame might be more persuasive for high-threat information. Study $8.1(\mathrm{~N}=98)$ used framed information advocating physical activity and found that gain-framed information resulted in higher ratings of information acceptance, particularly when the information was of a low-threat nature. Study 8.2 $(\mathrm{N}=99)$ used framed messages advocating a reduction in alcohol intake and found that gain-framed information resulted in more positive attitudes, but only when the information was of a low-threat nature. No advantage of loss frames was found in the high-threat condition. Results suggest that the effects of gainand loss-framed messages are dependent on the nature of the information that is conveyed in the message.

In the final chapter, the general discussion, the results are discussed and integrated. Further, methodological and theoretical issues are discussed, as well as implications for future research and practice. We conclude that the persuasiveness of gain- and loss-framed messages can be mediated by positive and negative affect, and that self-efficacy and message threat can moderate the effects of framed messages. Also, to increase levels of information acceptance, gain-framed rather than loss-framed health-promoting information might be used. 


\section{Samenvatting}

Gezondheidsvoorlichting kan de voordelen van gezond gedrag benadrukken (winstframe) of de nadelen van ongezond gedrag (verliesframe). Op dit moment is het niet duidelijk of winstframes dan wel verliesframes effectiever zijn. Om deze vraag te beantwoorden hebben onderzoekers zich gericht op de functie van het aanbevolen gedrag. Hierbij wordt een onderscheid gemaakt tussen gedragingen die ertoe dienen om ziekte te voorkomen, zoals regelmatig bewegen en stoppen met roken (preventiegedrag) en gedragingen die ertoe dienen om ziekte te ontdekken, zoals borstzelfonderzoek en baarmoederhalsonderzoek (detectiegedrag). Volgens sommige auteurs beschouwen mensen preventiegedragingen als relatief veilig, omdat ze de kans op ziekte verkleinen. Detectiegedragingen daarentegen, worden door mensen als riskant gezien, omdat de kans bestaat dat er door detectiegedrag een ongewenste ziekte aan het licht komt. Detectiegedrag brengt dus potentiële nadelen met zich mee. Omdat Prospect Theorie stelt dat winstframes ervoor zorgen dat mensen risicomijdende keuzes maken, veronderstelt men dat winstframes effectiever zijn in het aansporen tot preventiegedrag. Preventiegedrag is immers relatief veilig. En omdat verliesframes ervoor zorgen dat mensen riskante keuzes maken, veronderstelt men verder dat verliesframes effectiever zijn in het aansporen tot 'riskant' detectiegedrag. Uit recente bevindingen blijkt echter dat deze redenering niet altijd opgaat. Om deze hypothese te toetsen hebben we daarom een eerste studie verricht naar de invloed van winsten verliesframes die mensen aansporen om meer aan lichaamsbeweging te doen. Aangezien lichaamsbeweging een preventiegedrag is, zou men, volgens de hierboven vermelde redenering, kunnen veronderstellen dat winstframes effectiever zijn dan verliesframes. De resultaten van deze studie worden beschreven in Hoofdstuk 2. Omdat het effect van winst- en verliesframes nog niet is onderzocht in combinatie met op maat gemaakte voorlichting, hebben we de deelnemers $(\mathrm{N}$ = 787) eerst een door computer software gegenereerd advies op maat gegeven, daarna hebben we de deelnemers informatie verschaft over de voordelen van lichaamsbeweging óf over de nadelen van weinig lichaamsbeweging. Vervolgens hebben we onderzocht of winst- en verliesframes verschillende effecten hebben op acceptatie van de informatie, attitude, intentie en gedrag. De resultaten lieten zien dat winstframes resulteerden in een sterkere intentie om aan lichaamsbeweging te doen dan verliesframes. Dit had echter geen significante toename in lichaamsbeweging tot gevolg, gemeten tijdens een nameting na 3 maanden. Wat betreft informatie acceptatie en attitude werd een klein, niet significant, voordeel van winstframes gevonden. Deze resultaten suggereren dat winstframes overtuigender zijn dan verliesframes als het gaat om het aansporen tot lichaamsbeweging, maar dat de verschillen tussen winst- en verliesframes klein zijn. 
Uit de inconsistente bevindingen in de literatuur en de resultaten van Hoofdstuk 2, die worden behandeld in deel 1 van dit proefschrift, kunnen we dus concluderen dat een onderscheid tussen preventie- en detectiegedrag niet voldoende is om de effecten van winst- en verliesframes te begrijpen. In dit proefschrift stellen we dat er twee manieren zijn om meer inzicht te krijgen in de effecten van 'framing'. Ten eerste is het belangrijk om de onderliggende mechanismen van winst- en verliesframes te beschrijven. Ten tweede is het belangrijk om inzicht te krijgen in de omstandigheden waaronder winst- of verliesframes effectiever zijn. Overeenkomstig deze redenering behandelen we in deel 2 van dit proefschrift de onderliggende mechanismen van framing effecten (Hoofdstuk 3 en 4). De hypothese hierbij is dat positieve en negatieve gevoelens als reactie op geframede gezondheidsvoorlichting de effecten van framing kunnen mediëren. In deel 3 proberen we modererende variabelen te identificeren die de effecten van framing kunnen beïnvloeden, in het bijzonder de ervaren eigeneffectiviteit (Hoofdstuk 5, 6 en 7) en de mate waarin de gezondheidsinformatie bedreigend is (Hoofdstuk 8).

In Hoofdstuk 3 en 4 rapporteren we de resultaten van drie studies die de mediërende rol van positieve en negatieve gevoelens onderzoeken. Eerder onderzoek heeft laten zien dat, wanneer overredende boodschappen positieve gevoelens tot gevolg hebben, deze positieve gevoelens kunnen leiden tot een positieve attitude ten opzichte van de boodschap en een positieve attitude ten opzichte van het aanbevolen gedrag. Dit overeenkomstige effect op de attitude ten aanzien van de boodschap en het gedrag dat in de boodschap wordt aanbevolen staat bekend als de 'affect transfer hypothese'. Ook is gebleken dat negatieve gevoelens een overredende werking kunnen hebben. Uit de literatuur over angstaanjagende voorlichting blijkt bijvoorbeeld dat angst onder voorwaarden de effectiviteit van gezondheidsvoorlichting kan vergroten. Onze hypothese is nu dat zowel positieve gevoelens ten gevolge van winstframes, als negatieve gevoelens ten gevolge van verliesframes kunnen bijdragen aan de effectiviteit van gezondheidsvoorlichting. Als ze dat in gelijke mate doen ontstaat er een situatie waarin winst- en verliesframes even effectief zijn. Dit kan verklaren waarom eerdere studies geen verschillen tussen winst- en verliesframes hebben gevonden. In Studie 3.1, hebben we 100 deelnemers geframede informatie voorgelegd over lichaamsbeweging. De resultaten lieten zien dat winstframes resulteerden in een grotere mate van informatie acceptatie dan verliesframes en dat dit effect gemediëerd werd door positieve gevoelens. De resultaten van Studie 3.2, waarin we 129 deelnemers geframede informatie gaven over een zoutarm dieet, lieten zien dat winstframes resulteerden in hogere informatie acceptatie en een marginaal positievere attitude. Ook dit effect werd gemediëerd door positieve gevoelens. Ook lieten de resultaten zien dat verliesframes leidden tot meer negatieve gevoelens en dat deze negatieve gevoelens de intentie om een zoutarm dieet te volgen versterkten. In Hoofdstuk 4 werden deze resultaten gerepliceerd, met één uitzondering: posi- 
tieve gevoelens leverden geen bijdrage aan een positieve attitude. Deze resultaten suggereren dat positieve en negatieve gevoelens van groot belang kunnen zijn voor het effect van overredende boodschappen, en vooral behulpzaam kunnen zijn bij het in kaart brengen van de onderliggende mechanismen van framing effecten. De resultaten suggereren ook dat winst- en verliesframes via verschillende paden overredend kunnen zijn. Winstframes beïnvloeden de ontvanger door middel van de overredende werking van positieve gevoelens, terwijl verliesframes de ontvanger beïnvloeden door middel van de motiverende werking van negatieve gevoelens.

In Hoofdstuk 5 onderzochten we de invloed van eigeneffectiviteit op de effecten van geframede anti-rook boodschappen onder 539 volwassen rokers. Eerder onderzoek heeft laten zien dat bedreigende gezondheidsbevorderende boodschappen effectief kunnen zijn, op voorwaarde dat ontvangers het idee hebben dat ze het gevaar kunnen afwenden. Omdat verliesframes bedreigender zijn dan winstframes, zou dit ook voor verliesframes kunnen gelden. Onze hypothese is dat verliesframes effectief kunnen zijn, op voorwaarde dat ontvangers een hoge eigeneffectiviteit hebben om het aanbevolen gedrag uit te voeren. De resultaten van Studie 5 laten zien dat deelnemers met een hoge eigeneffectiviteit om te stoppen met roken een sterkere intentie om te stoppen met roken hadden na het lezen van verliesframes dan na het lezen van winstframes en na het lezen van geen informatie. Voor deze deelnemers was een winstframe niet effectiever dan het lezen van geen informatie. Voor deelnemers met een lage eigeneffectiviteit waren er geen verschillen in intentie tussen de winstframe-, verliesframe- en controle conditie. De resultaten suggereren dat eigeneffectiviteit de effecten van framing kan modereren.

In Hoofdstuk 6 rapporteren we de resultaten van een studie waarin werd onderzocht of de modererende werking van eigeneffectiviteit, gevonden in Studie 5, kon worden gerepliceerd. De resultaten van Studie 5 lieten zien dat eigeneffectiviteit een modererend effect had op de effecten van geframede boodschappen over roken, een preventiegedrag. Om de generaliseerbaarheid van onze resultaten te vergroten, en omdat verschillende auteurs veronderstellen dat framing verschillende effecten heeft bij preventie- en detectiegedragingen, hebben we in Studie 6 gebruik gemaakt van geframede boodschappen die aansporen tot huidzelfonderzoek. De resultaten van deze studie, onder 124 studenten, lieten zien dat verliesframes resulteerden in een sterkere intentie dan winstframes voor deelnemers met een hoge eigeneffectiviteit. Voor mensen met een lage eigeneffectiviteit waren er geen verschillen tussen winst- en verliesframes. Deze resultaten suggereren, net als de resultaten van Studie 5, dat eigeneffectiviteit een belangrijke invloed heeft op de effecten van framing. Voor gezondheidsvoorlichting die tot doel heeft om mensen aan te sporen tot stoppen met roken of huid zelfonderzoek kunnen verliesframes overtuigender zijn dan winstframes, maar alleen voor mensen met een hoge eigeneffectiviteit. 
De resultaten van Studie 5 en 6 suggereren dat eigeneffectiviteit de effecten van geframede boodschappen kan beïnvloeden. Deze twee studies hadden echter twee belangrijke beperkingen. Ten eerste maakten we gebruik van zelfrapportage maten van eigeneffectiviteit. Wanneer we experimentele manipulaties hadden gebruikt, zouden we met meer zekerheid uitspraken kunnen doen over de causaliteit van het effect. Ten tweede hebben we in deze studies geen gedrag gemeten, waardoor het onmogelijk is om vast te stellen of eigeneffectiviteit het effect van framing op gedrag kan beïnvloeden. In Hoofdstuk 7 hebben we daarom onderzocht of eigeneffectiviteit wederom de effecten van framing kon beïnvloeden, ditmaal gebruik makend van een experimentele manipulatie van eigeneffectiviteit en een nameting om gedrag te meten. In totaal 575 volwassenen, geworven via een internet panel, hebben meegedaan aan Studie 7. De helft van de deelnemers ontving eigeneffectiviteit bevorderende informatie, de ander helft kreeg deze informatie niet. Na deze manipulatie van eigeneffectiviteit ontvingen de deelnemers informatie over de gevolgen van zout eten. Voor de helft van de deelnemers was deze informatie geframed in termen van winst, de andere helft van de deelnemers ontving een verliesframe. De resultaten van een nameting na drie weken waren in overeenstemming met onze hypothese: verliesframes waren effectiever dan winstframes, maar alleen voor deelnemers met een hoge eigeneffectiviteit. In Studie 7 vonden we echter niet de veronderstelde interactie voor de uitkomstmaat intentie, in tegenstelling tot in Studie 5 en 6.

In Hoofdstuk 8 rapporteren we de resultaten van twee studies die onderzochten of de effecten van winst- en verliesframes verschilden tussen bedreigende en niet bedreigende informatie. De hypothese was dat winstframes overtuigender zouden zijn voor informatie die niet bedreigend is, terwijl verliesframes overtuigender is voor informatie die bedreigend is. In Studie $8.1(\mathrm{~N}=98)$ gebruikten we geframede informatie over lichaamsbeweging en vonden we dat winstframes resulteerden in een hogere mate van informatie acceptatie dan verliesframes, vooral wanneer de informatie niet bedreigend is. In Studie $8.2(\mathrm{~N}=$ 99) gebruikten we geframede informatie over alcoholgebruik en vonden we dat winstframes resulteerden in een positievere attitude dan verliesframes, maar alleen bij niet-bedreigende informatie. We vonden geen grotere effectiviteit van verliesframes voor de bedreigende informatie. Deze resultaten suggereren dat de effecten van winst- en verliesframes afhankelijk zijn van de inhoud van de informatie.

In het laatste hoofdstuk, de Algemene Discussie, bespreken we de resultaten van alle studies. Verder worden methodologische en theoretische kwesties behandeld en komen ook implicaties voor de praktijk van de gezondheidsvoorlichting aan bod. We concluderen dat de overtuigingskracht van winst- en verliesframes gemediëerd kan worden door positieve en negatieve gevoelens, en dat eigeneffectiviteit en dreiging de effecten van framing kunnen modereren. Ook 
concluderen we dat winstframes kunnen worden gebruikt om informatie acceptatie te vergroten. 



\section{Thank you}

They say no man is an island, but being a PhD-student sometimes makes you feel like one. In these final pages of my thesis, I would like to thank everybody who in the last couple of years helped me to think of myself as a peninsula.

First, I would like to thank Hein de Vries en Rob Ruiter, who gave me the opportunity to work on this project and profit from their expertise. Furthermore, I would like to thank Marieke Werrij for our constructive and inspirational meetings (also thanks to Rob Ruiter once again) and for co-authoring most of the chapters in this thesis.

Thank you ... all colleagues at the Department of Health Promotion. Without wanting to sell anyone short I want to mention Fam te Poel (thanks for social support), Chris Smerecnik (thanks for discussing science) and Bilbo Schickenberg (thanks for discussing ... well ... other things).

I would furthermore like to thank my parents for their love, warmth, intelligence, sound advice and optimistic outlook.

Finally ... Thank you, Maartje. You have brightened up my days during the last couple of years and I am grateful for every day I can spend with you, growing more and more confident that they will never ever find a cure for love. 



\section{Jonathan van 't Riet}

1980: Birth (January 12)

1998: Graduation at 'Gymnasium Celeanum', Zwolle

2004: Master's degree in Psychology, University of Groningen

2005: Research assistant, Department of Health Promotion, Maastricht University

2006: PhD-student, Department of Health Promotion, Maastricht University.

2009: Researcher in Consumer Behaviour, Agricultural Economics Research Institute, Wageningen University and Research Centre 

The "Kurt Lewin Institute Dissertation Series" started in 1997. Since 2007 the following dissertations have been published:

2007-1: Nils Jostmann: When the going gets tough... How action versus state orientation moderates the impact of situational demands on cognition, affect, and behavior

2007-2: Belle Derks: Social identity threat and performance motivation: The interplay between ingroup and outgroup domains

2007-3: Helma van den Berg: Feeling and Thinking in Attitudes

2007-4: Karin C.A. Bongers: You can't always get what you want! Consequences of success and failure to attain unconscious goals

2007-5: Lotte Scholten: Motivation matters: Motivated information processing in group and individual decision-making

2007-6: Debra Trampe: Social influence: Social comparison, construal, and persuasion processes

2007-7: Clemens Wenneker: Processes underlying biased language use

2007-8: Yaël de Liver: Ambivalence: on the how and when of attitudinal conflict

2007-9: Erik de Kwaadsteniet: Uncertainty in social dilemmas

2007-10: Hugo Alberts: Processes of self-control and ego depletion

2007-11: Loran Nordgren: Thinking about Feeling: The Nature and Significance of the Hot/Cold Empathy Gap

2007-12: Stefan Thomas Mol: Crossing Borders with Personnel Selection from expatriates to multicultural teams

2007-13: Hilbrand Oldenhuis: I know what they think about us: Metaperceptions and intergroup relations

2007-14: Arnaud Wisman: New Directions in Terror Management Theory

2007-15: Gert Homsma: Making Errors Worthwhile: Determinants of Constructive Error Handling

2007-16: Elianne van Steenbergen: Work-Family Facilitation: A Positive Psychological Perspective on Role Combination

2007-17: Unna Danner: By Force of Habit: On the Formation and Maintenance of Goal-Directed Habits

2007-18: Maureen Tumewu: The Social Psychology of Gender Differences and Procedural Justice in Close Relationships

2007-19: Wokje Abrahamse: Energy conservation through behavioral change: Examining the effectiveness of a tailor-made approach

2008-1: Marijke van Putten: Dealing with missed opportunities. The causes and boundary conditions of inaction inertia

2008-2: Marjolein Maas: Experiential Social Justice Judgment Processes

2008-3: Lonneke de Meijer: Ethnicity effects in police officer selection: Applicant, assessor, and selection-method factors 
2008-4: Frederike Zwenk: Voice by Representation

2008-5: Margreet Reitsma: The Impact of Linguistically Biased Messages on Involved Receivers

2008-6: Marcus Maringer: Feeling one thing, seeing another: Emotion comparison effects in person judgments

2008-7: Hanneke Heinsman: The competency concept revealed: Its nature, relevance, and practice

2008-8: Joris Lammers: Toward a more social social psychology of power

2008-9: Daniël Fockenberg: Between Good and Evil: Affective Priming in Dynamic Context

2008-10: Arne van den Bos: Why we stereotype influences how we stereotype: self-enhancement and comprehension effects on social perception

2008-11: Lidewij Niezink: Considering Others in Need: On Altruism, Empathy and Perspective Taking

2008-12: Aad Oosterhof: Better together: Antecedents and consequences of perceived expertise dissimilarity and perceived expertise complementarity in teams

2008-13: Femke ten Velden: Negotiation in dyads and groups: The effects of social and epistemic motives

2008-14: Maike Wehrens: How did YOU do? Social comparison in secondary education

2008-15: Kyra Luijters: Making Diversity Bloom: Coping Effectively with Cultural Differences at Work

2008-16: Ilona de Hooge: Moral emotions in decision making: Towards a better understanding of shame and guilt

2008-17: Lindred L. Greer: Team Composition and Conflict: The Role of Individual Differences

2008-18: Sezgin Cihangir: The Dark Side of Subtle Discrimination: How targets respond to different forms of discrimination

2008-19: Giel Dik: On the contagiousness of others' goals: The role of perceiving effort

2008-20: Lotte van Dillen: Dealing with negative feelings: The role of working memory in emotion regulation

2008-21: Marijn Poortvliet: Information exchange examined: An interpersonal account of achievement goals

2008-22: Sjoerd Pennekamp: Dynamics of disadvantage: Uncovering the role of group-based anger

2008-23: Chris Reinders Folmer: Cooperation and communication: Plastic goals and social roles

2009-1: Marijke Leliveld: Ethics in Economic Decision-Making

2009-2: Monique Pollmann: Accuracy and Bias in Person Perception

2009-3: Krispijn Faddegon: Regulatory Focus in Group Contexts 
2009-4: Lieven Brebels: Mirror, mirror on the wall... Procedural fairness as an evaluative and regulatory looking-glass self

2009-5: Daphne Wiersema: Taking it personally: Self-esteem and the protection of self-related attitudes

2009-6: Judith D.M. Grob: Dial E for Emotion: Context and Consequences of Emotion Regulation

2009-7: Katherine Stroebe: Is this about me? Responding to subtle discrimination - beyond an individual versus group perspective

2009-8: Menno Vos: Identity patterns in diverse work groups: Improving social integration outcomes through relational identities

2009-9: Lennart Renkema: Facing Death Together: Understanding The Consequences of Mortality Threats

2009-10: Michael Vliek: Group-based social comparison processes: An intragroup level of analysis

2009-11: Karlijn Massar: Unconscious rivals: The automatic evaluation of rivals in jealousy-evoking situations

2009-12: Bart Terwel: Origins and consequences of public trust: Towards an understanding of public acceptance of carbon dioxide capture and storage

2009-13: Emma ter Mors: Dealing with information about complex issues: The role of source perceptions

2009-14: Martijn Veltkamp: On the Instigation of Implicit Motivation: How Deprivation and Positive Affect Cause Motivated Behavior

2009-15: Marret K. Noordewier: Consistency and the unexpected

2009-16: Sytske van der Velde: Imitation of Emotion: How meaning affects the link between imitation and liking

2009-17: Jacomijn Hofstra: Attaching Cultures: The role of attachment styles in explaining majority members' acculturation attitudes

2009-18: Jacqueline Tanghe: Affect in Groups: Convergence, Conditions and Consequences

2009-19: Anne Marike Lokhorst: Using Commitment to Improve Environmental Quality

2009-20: Jonathan van 't Riet: Framing Health Communication Messages 